\title{
Computational Cosmology: From the Early Universe to the Large Scale Structure
}

\author{
Peter Anninos \\ University of California \\ Lawrence Livermore National Laboratory \\ 7000 East Ave. \\ Livermore, CA 94550-9234 \\ U.S.A. \\ email: anninos1@llnl.gov \\ Accepted on 15 February 2001 \\ Published on 20 March 2001 \\ http://www.livingreviews.org/lrr-2001-2 \\ Living Reviews in Relativity \\ Published by the Max Planck Institute for Gravitational Physics \\ Albert Einstein Institute, Germany
}

\begin{abstract}
In order to account for the observable Universe, any comprehensive theory or model of cosmology must draw from many disciplines of physics, including gauge theories of strong and weak interactions, the hydrodynamics and microphysics of baryonic matter, electromagnetic fields, and spacetime curvature, for example. Although it is difficult to incorporate all these physical elements into a single complete model of our Universe, advances in computing methods and technologies have contributed significantly towards our understanding of cosmological models, the Universe, and astrophysical processes within them. A sample of numerical calculations (and numerical methods) applied to specific issues in cosmology are reviewed in this article: from the Big Bang singularity dynamics to the fundamental interactions of gravitational waves; from the quark-hadron phase transition to the large scale structure of the Universe. The emphasis, although not exclusively, is on those calculations designed to test different models of cosmology against the observed Universe.
\end{abstract}

(C) Max Planck Society and the authors.

Further information on copyright is given at http://relativity.livingreviews.org/Info/Copyright/

For permission to reproduce the article please contact livrev@aei.mpg.de. 


\section{How to cite this article}

Owing to the fact that a Living Reviews article can evolve over time, we recommend to cite the article as follows:

Peter Anninos,

"Computational Cosmology: From the Early Universe to the Large Scale Structure", Living Rev. Relativity, 4, (2001), 2. [Online Article]: cited [<date $>$ ], http://www.livingreviews.org//rr-2001-2

The date given as $<$ date $>$ then uniquely identifies the version of the article you are referring to. 


\section{Article Revisions}

Living Reviews supports two different ways to keep its articles up-to-date:

Fast-track revision A fast-track revision provides the author with the opportunity to add short notices of current research results, trends and developments, or important publications to the article. A fast-track revision is refereed by the responsible subject editor. If an article has undergone a fast-track revision, a summary of changes will be listed here.

Major update A major update will include substantial changes and additions and is subject to full external refereeing. It is published with a new publication number.

For detailed documentation of an article's evolution, please refer always to the history document of the article's online version at http://www. livingreviews.org/lrr-2001-2.

14 April 2005: Fast-track revision to include recent developments. 37 new references have been added. Besides changes listed in detail, grammatical changes have been scattered throughout the article.

Page 8: Extended discussion to include a more complete chronology.

Page 8: Figure 1 added.

Page 9: Added a couple paragraphs to stress the dubious extension of the standard model to the early universe, added references for the classification of anisotropic cosmologies.

Page 14: Added references on early numerical simulations.

Page 14: Added references on BLK oscillations.

Page 18: Extended subsection to add several more references, and to discuss a recent paper on the stability of the phase fronts.

Page 18: Paragraphs added.

Page 18: Figures 5, 6, 7 added.

Page 23: Restructured section by inserting new subsubsection, moved subsubsection on Regge calculus model to appendix.

Page 24: Subsubsection added, new references included.

Page 25: Completely rewrote and reorganized section, added new references and a more detailed discussion on various anisotropy contributions.

Page 25: New subsubsection added.

Page 27: New subsubsection added.

Page 33: Added paragraph.

Page 34: Added paragraph which discusses relatively new references on model comparisons.

Page 39: Slightly modified summary section

Page 45: Slightly modified paragraph, reference added.

Page 46: Added paragraphs, expanded discussion to include mention of a new paper which uses NOCD methods for modeling ideal gases, and which compares artificial viscosity with conserving numerical methods. 
Page 47: New section added to include the stress tensor for nonideal fluids with shear stress.

Page 49: Reorganized section by adding new subsubsection, added example of a typical chemical network useful for primordial cosmology.

Page 51: Reorganized section by adding new subsubsection, added reference. 


\section{Contents}

1 Introduction $\quad 7$

2 Background $\quad 8$

2.1 A brief chronology . . . . . . . . . . . . . . . . . . . 8

2.2 The standard model . . . . . . . . . . . . . . . . . . . . . . . 9

3 Relativistic Cosmology 13

3.1 Singularities . . . . . . . . . . . . . . . . . . . . . . . 13

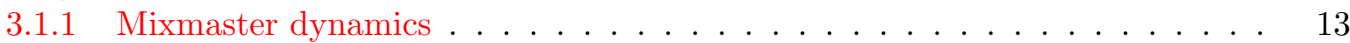

3.1 .2 AVTD vs. BLK oscillatory behavior . . . . . . . . . . . . . . 14

3.2 Inflation . . . . . . . . . . . . . . . . . . . . . . 15

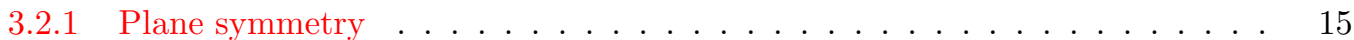

3.2.2 Spherical symmetry . . . . . . . . . . . . . . . . . . . . . . . . . . . . . .

3.2 .3 Bianchi V . . . . . . . . . . . . . . . . . . . . . . . . . . . . . . . .

3.2.4 Gravitational waves + cosmological constant . . . . . . . . . . . 16

3.2.5 3D inhomogeneous spacetimes . . . . . . . . . . . . . . . 16

3.3 Chaotic scalar field dynamics . . . . . . . . . . . . . . . . . . . . . . . . . . . . . . . . . . . . .

3.4 Quark-hadron phase transition . . . . . . . . . . . . . . . . . 18

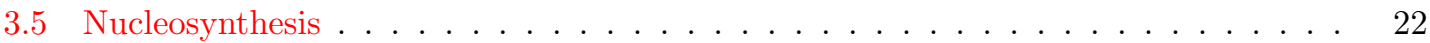

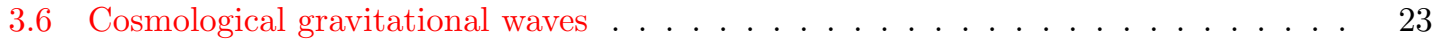

3.6.1 Planar symmetry . . . . . . . . . . . . . . . . . . 23

3.6.2 Multi-dimensional vacuum cosmologies . . . . . . . . . . . . . . . 24

4 Physical Cosmology $\quad 25$

4.1 Cosmic microwave background . . . . . . . . . . . . . . . . . . . . . . . . . . . . . . . .

4.1 Primordial black body effects . . . . . . . . . . . . . . . 25

4.1 .2 Primary anisotropies . . . . . . . . . . . . . . . . . . . . . . . . . . . . . . . . .

4.1 .3 Secondary anisotropies . . . . . . . . . . . . . . . . . . 27

4.1.4 Computing CMBR anisotropies with ray-tracing methods . . . . . . . . . 29

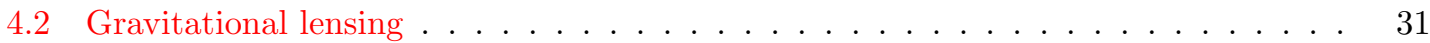

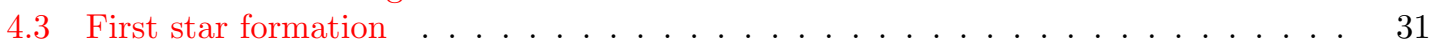

4.4 Ly $\alpha$ forest . . . . . . . . . . . . . . . . . . . . . . . . . . . . 33

4.5 Galaxy clusters . . . . . . . . . . . . . . . . . . . . . . . . . . . . . . . . . . . . . .

4.5.1 Internal structure . . . . . . . . . . . . . . . . . . . . . . . . . . . . . . . . . . . . .

4.5.2 Number density evolution . . . . . . . . . . . . . . . . . . . . . . . . . . . . . . . .

4.5.3 X-ray luminosity function . . . . . . . . . . . . . . . . . . 36

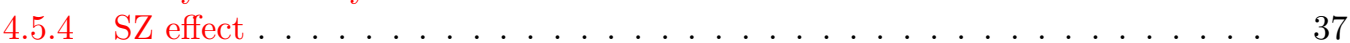

4.6 Cosmological sheets . . . . . . . . . . . . . . . . . . . 37

$\begin{array}{llr}5 & \text { Summary } & 39\end{array}$

6 Appendix: Basic Equations and Numerical Methods $\quad 40$

6.1 The Einstein equations . . . . . . . . . . . . . . . . . . . . . . . 40

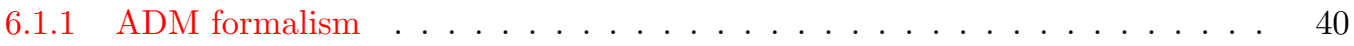

6.1 .2 Kinematic conditions . . . . . . . . . . . . . . . . . . 41

6.1 .3 Symplectic formalism . . . . . . . . . . . . . . . . . . . . . 42

6.1 .4 Regge calculus model . . . . . . . . . . . . . . . . . . . . . 42

6.2 Sources of matter . . . . . . . . . . . . . . . . . . . . . . . . . . . . . . . . . . . . . . . . . . . .

6.2.1 Cosmological constant . . . . . . . . . . . . . . . 43 
6.2 .2 Scalar field . . . . . . . . . . . . . . . . . . . . . . . . . 43

6.2 .3 Collisionless dust . . . . . . . . . . . . . . . . . . . . 44

6.2 .4 Ideal gas . . . . . . . . . . . . . . . . . . . . . . . 45

6.2 .5 Imperfect fluid . . . . . . . . . . . . . . . . . . . . 47

6.3 Constrained nonlinear initial data . . . . . . . . . . . . . . . 47

6.4 Newtonian limit . . . . . . . . . . . . . . . . . . . . . . . . 48

6.4.1 Dark and baryonic matter equations . . . . . . . . . . . . . . 48

6.4 .2 Primordial chemistry . . . . . . . . . . . . . . . . . . . . . . 49

6.4 .3 Numerical methods . . . . . . . . . . . . . . . . . . . . . . . 51

6.4 .4 Linear initial data . . . . . . . . . . . . . . . . . . . . . . . . 51

$\begin{array}{ll}\text { References } & 53\end{array}$ 


\section{Introduction}

Numerical investigations of cosmological spacetimes can be categorized into two broad classes of calculations, distinguished by their computational goals: (i) geometrical and mathematical principles of cosmological models, and (ii) physical and astrophysical cosmology. In the former, the emphasis is on the geometric framework in which astrophysical processes occur, for example cosmological expansion, topological singularities, geometrodynamics in general, and classification characteristics or invariants of the many models allowed by the theory of general relativity. In the latter, the emphasis is on the cosmological and astrophysical processes in the real or observable Universe, and the quest to determine the model which best describes our Universe. The former is pure in the sense that it concerns the fundamental nonlinear behavior of the Einstein equations and the gravitational field. The latter is more complex as it addresses the composition, organization, and dynamics of the Universe from the small scales (fundamental particles and elements) to the large (galaxies and clusters of galaxies). However the distinction is not always so clear, and geometric effects in the spacetime curvature can have significant consequences for the evolution and observation of matter distributions.

Any comprehensive model of cosmology must therefore include nonlinear interactions between different matter sources and spacetime curvature. A realistic model of the Universe must also cover large dynamical spatial and temporal scales, extreme temperature and density distributions, and highly dynamic atomic and molecular matter compositions. In addition, due to all the varied physical processes of cosmological significance, one must draw from many disciplines of physics to model curvature anisotropies, gravitational waves, electromagnetic fields, nucleosynthesis, particle physics, hydrodynamic fluids, etc. These phenomena are described in terms of coupled nonlinear partial differential equations and must be solved numerically for general inhomogeneous spacetimes. The situation appears extremely complex, even with current technological and computational advances. As a result, the codes and numerical methods that have been developed to date are designed to investigate very specific problems with either idealized symmetries or simplifying assumptions regarding the metric behavior, the matter distribution/composition or the interactions among the matter types and spacetime curvature.

It is the purpose of this article to review published numerical cosmological calculations addressing problems from the very early Universe to the present; from the purely geometrical dynamics of the initial singularity to the large scale structure of the Universe. There are three major sections: Section 2 where a brief overview is presented of various defining events occurring throughout the history of our Universe and in the context of the standard model, Section 3 where reviews of early Universe and relativistic cosmological calculations are presented, and Section 4 which focuses on structure formation in the post-recombination epoch and on testing cosmological models against observations. Following the summary paragraphs in Section 5, an appendix in Section 6 presents the basic Einstein equations, kinematic considerations, matter source equations with curvature, and the equations of perturbative physical cosmology on background isotropic models. References to numerical methods are also supplied and reviewed for each case. 


\section{Background}

\subsection{A brief chronology}

With current observational constraints, the physical state of our Universe, as understood in the context of the standard or Friedman-Lemaitre-Robertson-Walker (FLRW) model, can be crudely extrapolated back to $\sim 10^{-43}$ seconds after the Big Bang, before which the classical description of general relativity is expected to give way to a quantum theory of gravity. As the time-line in Figure 1 shows, the Universe was a plasma of relativistic particles at the earliest times consisting of quarks, leptons, gauge bosons, and Higgs bosons represented by scalar fields with interaction and symmetry regulating potentials.

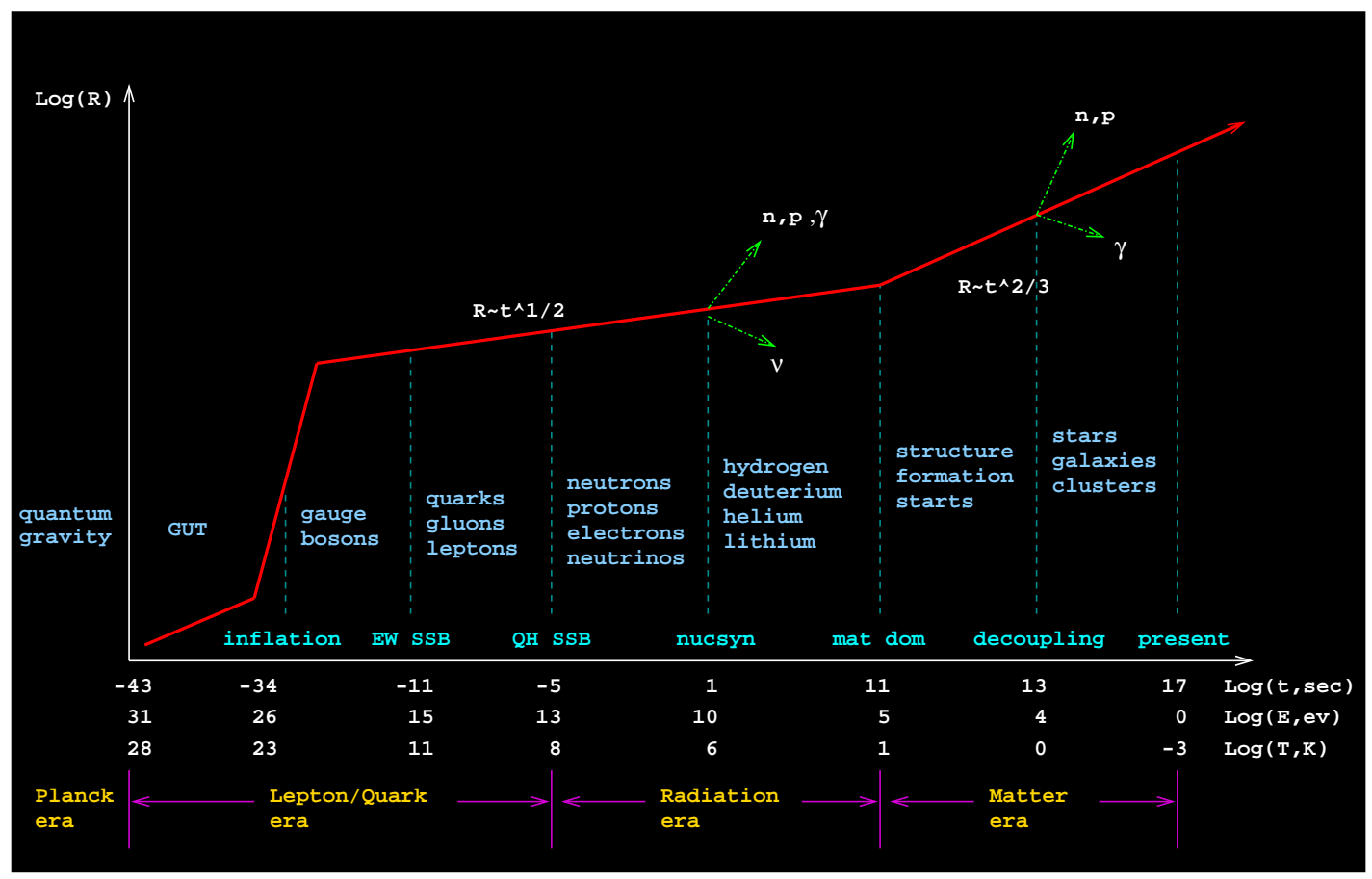

Figure 1: A historical time-line showing the major evolutionary stages of our Universe according to the standard model, from the earliest moments of the Planck era to the present. The horizontal axis represents logarithmic time in seconds (or equivalently energy in electron-Volts or temperature in Kelvin), and the solid red line roughly models the radius of the Universe, showing the different rates of expansion at different times: exponential during inflation, shallow power law during the radiation dominated era, and a somewhat steeper power law during the current matter dominated phase.

It is believed that several spontaneous symmetry breaking (SSB) phase transitions occured in the early Universe as it expanded and cooled, including the grand unification transition (GUT) at $\sim 10^{-34}$ s after the Big Bang in which the strong nuclear force split off from the weak and electromagnetic forces (this also marks an era of inflationary expansion and the origin of matter-antimatter asymmetry through baryon, charge conjugation, and charge + parity violating interactions and nonequilibrium effects); the electroweak (EW) SSB transition at $\sim 10^{-11} \mathrm{~s}$ when the weak nuclear force split from the electromagnetic force; and the chiral or quantum chromodynamic (QCD) symmetry breaking transition at $\sim 10^{-5} \mathrm{~s}$ during which quarks condensed into hadrons. The most

Living Reviews in Relativity

http: //www. livingreviews. org/lrr-2001-2 
stable hadrons (baryons, or protons and neutrons comprised of three quarks) survived the subsequent period of baryon-antibaryon annihilations, which continued until the Universe cooled to the point at which new baryon-antibaryon pairs could no longer be produced. This resulted in a large number of photons and relatively few surviving baryons. Topological defects, defined as stable configurations of matter in the symmetric (high temperature) phase, may persist after any of the phase transitions described above to influence the subsequent evolution of matter structures. The nature of the defects is determined by the phase transition and the symmetry properties of the matter, and some examples include domain walls, cosmic strings, monopoles, and textures.

A period of primordial nucleosynthesis followed from $\sim 10^{-2}$ to $\sim 10^{2}$ s during which light element abundances were synthesized to form $24 \%$ helium with trace amounts of deuterium, tritium, helium-3, and lithium. Observations of these relative abundances represent the earliest confirmation of the standard model. It is also during this stage that neutrinos (produced from proton-proton and proton-photon interactions, and from the collapse or quantum evaporation/annihilation of topological defects) stopped interacting with other matter, such as neutrons, protons, and photons. Neutrinos that existed at this time separated from these other forms of matter and traveled freely through the Universe at very high velocities, near the speed of light.

By $\sim 10^{11} \mathrm{~s}$, the matter density became equal to the radiation density as the Universe continued to expand, identifying the start of the current matter-dominated era and the beginning of structure formation. Later, at $\sim 10^{13} \mathrm{~s}\left(3 \times 10^{5} \mathrm{yr}\right)$, the free ions and electrons combined to form atoms, effectively decoupling the matter from the radiation field as the Universe cooled. This decoupling or post-recombination epoch marks the surface of last scattering and the boundary of the observable (via photons) Universe, and plays an important role in the history of the Cosmic Microwave Background Radiation (CMBR). Assuming a hierarchical Cold Dark Matter (CDM) structure formation scenario, the subsequent development of our Universe is characterized by the growth of structures with increasing size. For example, the first stars are likely to have formed at $t \sim 10^{8} \mathrm{y}$ from molecular gas clouds when the Jeans mass of the background baryonic fluid was approximately $10^{4} M_{\odot}$, as indicated in Figure 2. This epoch of pop III star generation is followed by the formation of galaxies at $t \sim 10^{9} \mathrm{yr}$ and subsequently galaxy clusters. Though somewhat controversial, estimates of the current age of our Universe range from 10 to $20 \mathrm{~Gy}$, with a presentday linear structure scale radius of about $8 h^{-1} \mathrm{Mpc}$, where $h$ is the Hubble parameter (compared to $2-3 \mathrm{Mpc}$ typical for the virial radius of rich galaxy clusters).

\subsection{The standard model}

The isotropic and homogeneous FLRW cosmological model has been so successful in describing the observable Universe that it is commonly referred to as the "standard model". Furthermore, and to its credit, the model is relatively simple so that it allows for calculations and predictions to be made of the very early Universe, including primordial nucleosynthesis at $10^{-2}$ seconds after the Big Bang, and even particle interactions approaching the Planck scale at $10^{-43} \mathrm{~s}$. At present, observational support for the standard model includes:

- the expansion of the Universe as verified by the redshifts in galaxy spectra and quantified by measurements of the Hubble constant $H_{0}=100 h \mathrm{~km} \mathrm{~s}^{-1} \mathrm{Mpc}^{-1}$, where $0.5 \leq h \leq 1$ is the Hubble constant;

- the deceleration parameter observed in distant galaxy spectra (although uncertainties about galactic evolution, intrinsic luminosities, and standard candles prevent an accurate estimate);

- the large scale isotropy and homogeneity of the Universe based on temperature anisotropy measurements of the microwave background radiation and peculiar velocity fields of galaxies (although the light distribution from bright galaxies is somewhat contradictory); 


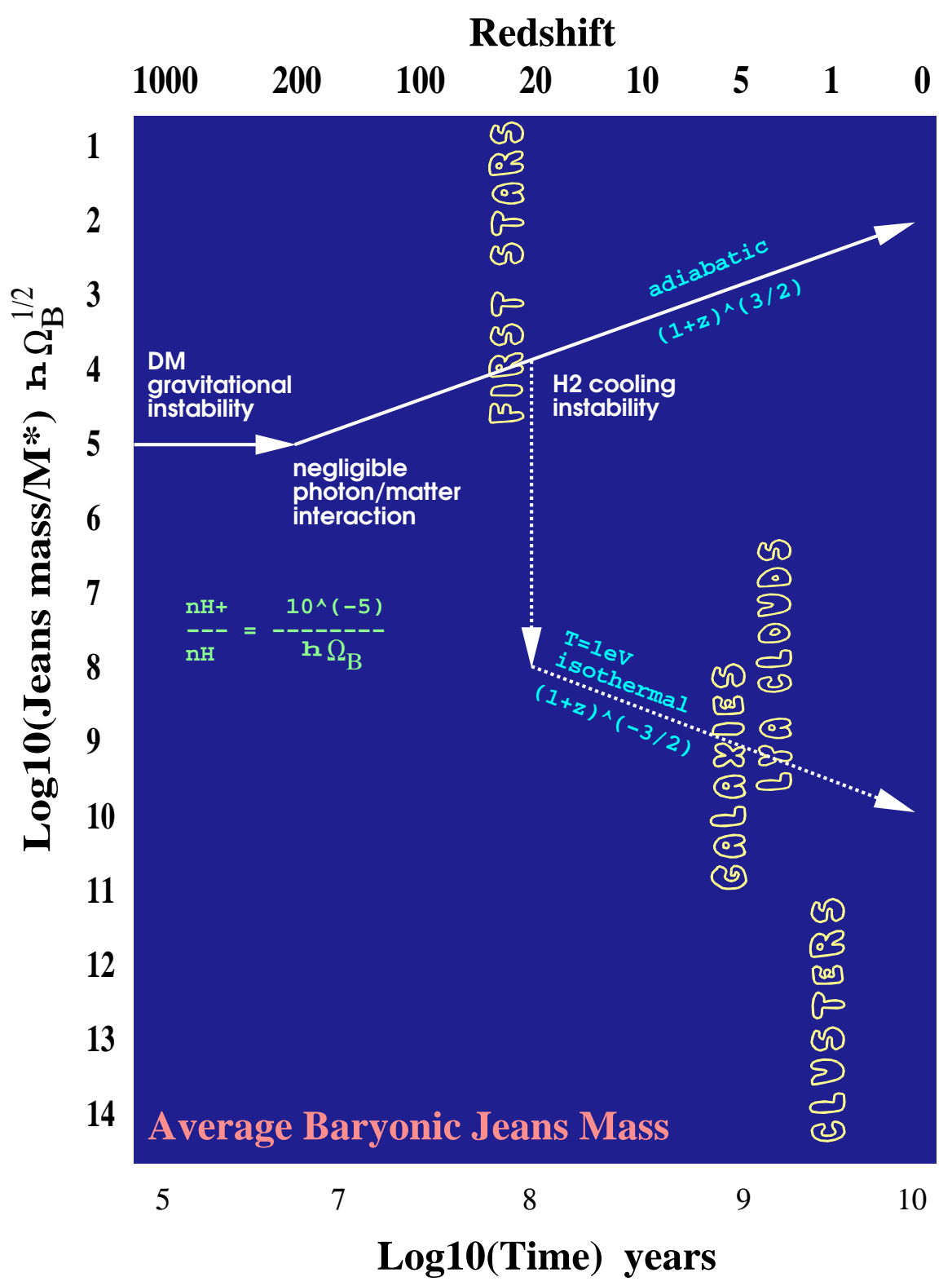

Figure 2: Schematic depicting the general sequence of events in the post-recombination Universe. The solid and dotted lines potentially track the Jeans mass of the average baryonic gas component from the recombination epoch at $z \sim 10^{3}$ to the current time. A residual ionization fraction of $n_{\mathrm{H}+} / n_{\mathrm{H}} \sim 10^{-4}$ following recombination allows for Compton interactions with photons to $z \sim 200$, during which the Jeans mass remains constant at $10^{5} M_{\odot}$. The Jeans mass then decreases as the Universe expands adiabatically until the first collapsed structures form sufficient amounts of hydrogen molecules to trigger a cooling instability and produce pop III stars at $z \sim 20$. Star formation activity can then reheat the Universe and raise the mean Jeans mass to above $10^{8} M_{\odot}$. This reheating could affect the subsequent development of structures such as galaxies and the observed Lya clouds.

Living Reviews in Relativity

http: //www . livingreviews . org/lrr-2001-2 
- the age of the Universe which yields roughly consistent estimates between the look-back time to the Big Bang in the FLRW model and observed data such as the oldest stars, radioactive elements, and cooling of white dwarf stars;

- the cosmic microwave background radiation suggests that the Universe began from a hot Big Bang and the data is consistent with a mostly isotropic model and a black body at temperature $2.7 \mathrm{~K}$;

- $C M B R$ precision measurements suggest best fit cosmological parameters in accord with the critical density standard model;

- the abundance of light elements such as ${ }^{2} \mathrm{H},{ }^{3} \mathrm{He},{ }^{4} \mathrm{He}$, and ${ }^{7} \mathrm{Li}$, as predicted from the FLRW model, is consistent with observations, provides a bound on the baryon density and baryonto-photon ratio, and is the earliest confirmation of the standard model;

- the present mass density, as determined from measurements of luminous matter and galactic rotation curves, can be accounted for by the FLRW model with a single density parameter $\left(\Omega_{0}\right)$ to specify the metric topology;

- the distribution of galaxies and larger scale structures can be reproduced by numerical simulations in the context of inhomogeneous perturbations of the FLRW models;

- the detection of dark energy from observations of supernovae is generally consistent with accepted FLRW model parameters and cold dark matter + cosmological constant numerical structure formation models.

Because of these remarkable agreements between observation and theory, most work in the field of physical cosmology (see Section 4) has utilized the standard model as the background spacetime in which the large scale structure evolves, with the ambition to further constrain parameters and structure formation scenarios through numerical simulations. The most widely accepted form of the model is described by a set of dimensionless density parameters which sum to

$$
\Omega_{b}+\Omega_{d}+\Omega_{\gamma}+\Omega_{\Lambda}=\Omega_{0},
$$

where the different components measure the present mean baryon density $\Omega_{\mathrm{b}}$, the dark matter density $\Omega_{\mathrm{d}}$, the radiation energy $\Omega_{\gamma}$, and the dark energy $\Omega_{\Lambda}$. The relative contributions of each source and their sum $\Omega_{0}$ (which determines the topological curvature of the model) remains one of the most important issues in modern computational and observational cosmology. The reader is referred to [104] for a more in-depth review of the standard model, and to [128, 154] for a summary of observed cosmological parameter constraints and best fit "concordance" models. Peebles and Ratra [133] provide a comprehensive literature survey and an excellent review of the standard model, cosmological tests, and the evidence for dark energy and the cosmological constant.

However, some important unanswered questions about the standard model concern the nature of the special conditions that produced an essentially geometrically flat Universe that is also homogeneous and isotropic to a high degree over large scales. In an affort to address these questions, it should be noted that many other cosmological models can be constructed with a late time behavior similar enough to the standard model that it is difficult to exclude them with absolute certainty. Consider, for example, the collection of homogeneous but arbitrarily anisotropic vacuum spacetimes known as the Bianchi models [141,69]. There are nine unique models in this family of cosmologies, ranging from simple Bianchi I models representing the Kasner class of spacetimes (the flat FLRW model, sometimes referred to as Type I-homogeneous, belongs to this group), to the more complex and chaotic Bianchi IX or Mixmaster model (which also includes the closed FLRW model, Type IX-homogeneous). Several of these models will be discussed in the first section 
on relativistic cosmology (Section 3) dealing pre-dominately with the early Universe, where the models differ the most.

Anisotropic solutions, as well as more general (and in some cases exact) inhomogeneous cosmological models with initial singularities, can isotropize through anisotropic damping mechanisms and adiabatic cooling by the expansion of the Universe to resemble the standard FLRW model at late times. Furthermore, if matter is included in these spacetimes, the effective energy of anisotropy, which generally dominates matter energy at early times, tends to become less important over time as the Universe expands. The geometry in these matter-filled anisotropic spacetimes thus evolves towards an isotropic state. Quantum mechanical effects have also been proposed as a possible anisotropy damping mechanism that takes place in the early Universe to convert vacuum geometric energy to radiation energy and create particles. All of this suggests that the early time behavior and effects of local and global geometry are highly uncertain, despite the fact that the standard FLRW model is generally accepted as accurate enough for the late time description of our Universe.

Further detailed information on homogeneous (including Bianchi) universes, as well as more general classes of inhomogeneous cosmological models can be found in [105, 158, 70].

Living Reviews in Relativity

http://www. livingreviews.org/lrr-2001-2 


\section{Relativistic Cosmology}

This section is organized to track the chronological events in the history of the early or relativistic Universe, focusing mainly on four defining moments: (i) the Big Bang singularity and the dynamics of the very early Universe, (ii) inflation and its generic nature, (iii) QCD phase transitions, and (iv) primordial nucleosynthesis and the freeze-out of the light elements. The late or post-recombination epoch is reserved to a separate Section 4.

\subsection{Singularities}

\subsubsection{Mixmaster dynamics}

Belinsky, Lifshitz, and Khalatnikov (BLK) [32, 33] and Misner [119] discovered that the Einstein equations in the vacuum homogeneous Bianchi type IX (or Mixmaster) cosmology exhibit complex behavior and are sensitive to initial conditions as the Big Bang singularity is approached. In particular, the solutions near the singularity are described qualitatively by a discrete map [30, 32] representing different sequences of Kasner spacetimes

$$
d s^{2}=-d t^{2}+t^{2 p_{1}} d x^{2}+t^{2 p_{2}} d y^{2}+t^{2 p_{3}} d z^{2},
$$

with time changing exponents $p_{i}$, but otherwise constrained by $p_{1}+p_{2}+p_{3}=p_{1}^{2}+p_{2}^{2}+p_{3}^{2}=1$. Because this discrete mapping of Kasner epochs is chaotic, the Mixmaster dynamics is presumed to be chaotic as well.

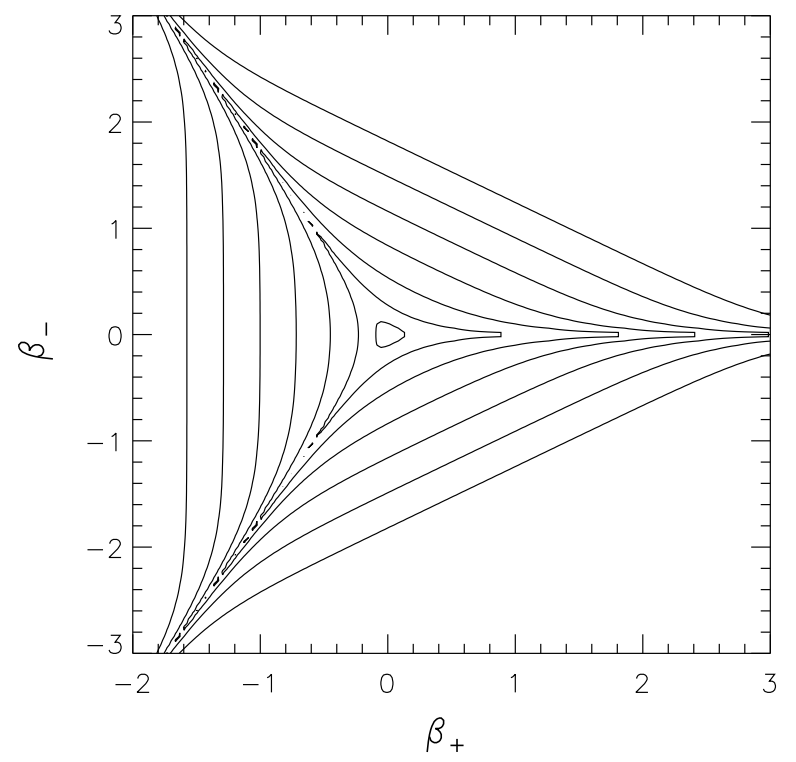

Figure 3: Contour plot of the Bianchi type IX potential $V$, where $\beta_{ \pm}$are the anisotropy canonical coordinates. Seven level surfaces are shown at equally spaced decades ranging from $10^{-1}$ to $10^{5}$. For large isocontours $(V>1)$, the potential is open and exhibits a strong triangular symmetry with three narrow channels extending to spatial infinity. For $V<1$, the potential closes and is approximately circular for $\beta_{ \pm} \ll 1$.

Mixmaster behavior can be studied in the context of Hamiltonian dynamics, with a Hamiltonian [120]

$$
2 \mathcal{H}=-p_{\Omega}^{2}+p_{+}^{2}+p_{-}^{2}+e^{4 \alpha}(V-1)
$$


and a semi-bounded potential arising from the spatial scalar curvature (whose level curves are plotted in Figure 3)

$$
V=1+\frac{1}{3} e^{-8 \beta_{+}}+\frac{2}{3} e^{4 \beta_{+}}\left[\cosh \left(4 \sqrt{3} \beta_{-}\right)-1\right]-\frac{4}{3} e^{-2 \beta_{+}} \cosh \left(2 \sqrt{3} \beta_{-}\right),
$$

where $e^{\alpha}$ and $\beta_{ \pm}$are the scale factor and anisotropies, and $p_{\alpha}$ and $p_{ \pm}$are the corresponding conjugate variables. A solution of this Hamiltonian system is an infinite sequence of Kasner epochs with parameters that change when the phase space trajectories bounce off the potential walls, which become exponentially steep as the system evolves towards the singularity.

Some of the earliest numerical simulations of this dynamical system were performed by Matzner, Shepley, and Warren [116], and Moser, Matzner and Ryan [123] who followed phase space trajectories and provided examples of solutions for various initial conditions and special cases. Several, more recent, numerical calculations of the equations arising from Equations (3) and (4) have indicated that the Liapunov exponents of the system vanish, in apparent contradiction with the discrete maps [53, 89], and putting into question the characterization of Mixmaster dynamics as chaotic. However, it has since been shown that the usual definition of the Liapunov exponents is ambiguous in this case as it is not invariant under time reparametrizations, and that with a different time variable one obtains positive exponents [35, 73]. Also, coordinate independent methods using fractal basin boundaries to map basins of attraction in the space of initial conditions indicates Mixmaster spacetimes to be chaotic [64].

Although BLK conjectured that local Mixmaster oscillations might be the generic behavior for singularities in more general classes of spacetimes [33], it is only recently that this conjecture has begun to be supported by numerical evidence (see Section 3.1 .2 and [37]).

\subsubsection{AVTD vs. BLK oscillatory behavior}

As noted in Section 3.1.1, an interesting and important issue in classical cosmology is whether or not the generic Big Bang singularity is locally of a Mixmaster or BLK type, with complex oscillatory behavior as the singularity is approached. Many of the Bianchi models, including the Kasner solutions (2), are characterized by either open or no potentials in the Hamiltonian framework [141], and exhibit essentially monotonic or Asymptotically Velocity Term Dominated (AVTD) behavior.

Considering inhomogeneous spacetimes, Isenberg and Moncrief [98] proved that the singularity in the polarized Gowdy model is AVTD type, as are more general polarized $T^{2}$ symmetric cosmologies [38]. Early numerical studies using symplectic methods confirmed AVTD behavior and found no evidence of BLK oscillations, even in $T^{3} \times R$ spacetimes with $U(1)$ symmetry [36] (although there were concerns about the solutions due to difficulties in resolving steep spatial gradients near the singularity [36], which were addressed later by Hern and Stewart [87] for the Gowdy $T^{3}$ models).

However, Weaver et al. [160] established the first evidence through numerical simulations that Mixmaster dynamics can occur in a class of inhomogeneous spacetimes which generalize the Bianchi type $\mathrm{VI}_{0}$ model with a magnetic field and two-torus symmetry. Berger and Moncrief [41, 42] also demonstrated that $U(1)$ symmetric vacuum cosmologies exhibit local Mixmaster dynamics consistent with the BLK conjecture, despite numerical difficulties in resolving steep gradients (which they managed by enforcing the Hamiltonian constraint and spatially averaging the solutions). Another more recent example supporting the BLK conjecture is provided by Garfinkle [79], who finds local oscillating behavior approaching the singularity in closed vacuum (but otherwise generic) spacetimes with no assumed symmetry in the initial data.

Living Reviews in Relativity

http://www. livingreviews.org/lrr-2001-2 


\subsection{Inflation}

The inflation paradigm is frequently invoked to explain the flatness $\left(\Omega_{0} \approx 1\right.$ in the context of the FLRW model) and nearly isotropic nature of the Universe at large scales, attributing them to an era of exponential expansion at about $10^{-34} \mathrm{~s}$ after the Big Bang. This expansion acts as a strong dampening mechanism to random curvature or density fluctuations, and may be a generic attractor in the space of initial conditions. An essential component needed to trigger this inflationary phase is a scalar or inflaton field $\phi$ representing spin zero particles. The vacuum energy of the field acts as an effective cosmological constant that regulates GUT symmetry breaking, particle creation, and the reheating of the Universe through an interaction potential $V(\phi)$ derived from the form of symmetry breaking that occurs as the temperature of the Universe decreases.

Early analytic studies focused on simplified models, treating the interaction potential as flat near its local maximum where the field does not evolve significantly and where the formal analogy to an effective cosmological constant approximation is more precise. However, to properly account for the complexity of inflaton fields, the full dynamical equations (see Section 6.2.2) must be considered together with consistent curvature, matter and other scalar field couplings. Also, many different theories of inflation and vacuum potentials have been proposed (see, for example, a recent review by Lyth and Riotto [113] and an introductory article by Liddle [111]), and it is not likely that simplified models can elucidate the full nonlinear complexity of scalar fields (see Section 3.3) nor the generic nature of inflation.

In order to study whether inflation can occur for arbitrary anisotropic and inhomogeneous data, many numerical simulations have been carried out with different symmetries, matter types and perturbations. A sample of such calculations is described in the following paragraphs.

\subsubsection{Plane symmetry}

Kurki-Suonio et al. [106] extended the planar cosmological code of Centrella and Wilson [59, 60] (see Section 3.6.1) to include a scalar field and simulate the onset of inflation in the early Universe with an inhomogeneous Higgs field and a perfect fluid with a radiation equation of state $p=\rho / 3$, where $p$ is the pressure and $\rho$ is the energy density. Their results suggest that whether inflation occurs or not can be sensitive to the shape of the potential $\phi$. In particular, if the shape is flat enough and satisfies the slow-roll conditions (essentially upper bounds on $\partial V / \partial \phi$ and $\partial^{2} V / \partial \phi^{2}$ [104] near the false vacuum $\phi \sim 0$ ), even large initial fluctuations of the Higgs field do not prevent inflation. They considered two different forms of the potential: a Coleman-Weinberg type with interaction strength $\lambda$ and distance between true and false vacua $\sigma$

$$
V(\phi)=\lambda \phi^{4}\left[\ln \left(\frac{\phi^{2}}{\sigma^{2}}\right)-\frac{1}{2}\right]+\frac{\lambda \sigma^{4}}{2},
$$

which is very flat near the false vacuum and does inflate; and a rounder " $\phi$ " type

$$
V(\phi)=\lambda\left(\phi^{2}-\sigma^{2}\right)^{2},
$$

which, for their parameter combinations, does not.

\subsubsection{Spherical symmetry}

Goldwirth and Piran [83] studied the onset of inflation with inhomogeneous initial conditions for closed, spherically symmetric spacetimes containing a massive scalar field and radiation field sources (described by a massless scalar field). In all the cases they considered, the radiation field damps quickly and only an inhomogeneous massive scalar field remains to inflate the Universe. They find that small inhomogeneities tend to reduce the amount of inflation and large initial inhomogeneities 
can even suppress the onset of inflation. Their calculations indicate that the scalar field must have "suitable" initial values (local conditions for which an equivalent homogeneous Universe will inflate) over a domain of several horizon lengths in order to trigger inflation.

\subsubsection{Bianchi V}

Anninos et al. [14] investigated the simplest Bianchi model (type V) background that admits velocities or tilt in order to address the question of how the Universe can choose a uniform reference frame at the exit from inflation, since the de Sitter metric does not have a preferred frame. They find that inflation does not isotropize the Universe in the short wavelength limit. However, if inflation persists, the wave behavior eventually freezes in and all velocities go to zero at least as rapidly as $\tanh \beta \sim R^{-1}$, where $\beta$ is the relativistic tilt angle (a measure of velocity), and $R$ is a typical scale associated with the radius of the Universe. Their results indicate that the velocities eventually go to zero as inflation carries all spatial variations outside the horizon, and that the answer to the posed question is that memory is retained and the Universe is never really de Sitter.

\subsubsection{Gravitational waves + cosmological constant}

In addition to the inflaton field, one can consider other sources of inhomogeneity, such as gravitational waves. Although linear waves in de Sitter space will decay exponentially and disappear, it is unclear what will happen if strong waves exist. Shinkai and Maeda [148] investigated the cosmic no-hair conjecture with gravitational waves and a cosmological constant $(\Lambda)$ in $1 \mathrm{D}$ plane symmetric vacuum spacetimes, setting up Gaussian pulse wave data with amplitudes $0.02 \Lambda \leq \max (\sqrt{I}) \leq 80 \Lambda$ and widths $0.08 l_{\mathrm{H}} \leq l \leq 2.5 l_{\mathrm{H}}$, where $I$ is the invariant constructed from the 3-Riemann tensor and $l_{\mathrm{H}}=\sqrt{3 / \Lambda}$ is the horizon scale. They also considered colliding plane waves with amplitudes $40 \Lambda \leq \max (\sqrt{I}) \leq 125 \Lambda$ and widths $0.08 l_{\mathrm{H}} \leq l \leq 0.1 l_{\mathrm{H}}$. They find that for any large amplitude or small width inhomogeneity in their parameter sets, the nonlinearity of gravity has little effect and the spacetime always evolves towards de Sitter.

\subsubsection{D inhomogeneous spacetimes}

The previous paragraphs discussed results from highly symmetric spacetimes, but the possibility of inflation remains to be established for more general inhomogeneous and nonperturbative data. In an effort to address this issue, Kurki-Suonio et al. [107] investigated fully three-dimensional inhomogeneous spacetimes with a chaotic inflationary potential $V(\phi)=\lambda \phi^{4} / 4$. They considered basically two types of runs: small and large scale. For the small scale runs, the grid dimensions were initially set equal to the Hubble length so the inhomogeneities are well inside the horizon and the dynamical time scale is shorter than the expansion or Hubble time. As a result, the perturbations oscillate and damp, while the field evolves and the spacetime inflates. For the large scale runs, the inhomogeneities are outside the horizon and they do not oscillate. They maintain their shape without damping and, because larger values of $\phi$ lead to faster expansion, the inhomogeneity in the expansion becomes steeper in time since the regions of large $\phi$ and high inflation stay correlated. Both runs produce enough inflation to solve the flatness problem.

\subsection{Chaotic scalar field dynamics}

Many studies of cosmological models generally reduce complex physical systems to simplified or even analytically integrable systems. In sufficiently simple models the dynamical behavior (or fate) of the Universe can be predicted from a given set of initial conditions. However, the Universe is composed of many different nonlinear interacting fields including the inflaton field which can exhibit complex chaotic behavior. For example, Cornish and Levin [63] consider a homogenous isotropic

Living Reviews in Relativity

http: //www. livingreviews. org/lrr-2001-2 
closed FLRW model with various conformal and minimally coupled scalar fields (see Section 6.2.2). They find that even these relatively simple models exhibit chaotic transients in their early preinflationary evolution. This behavior in exiting the Planck era is characterized by fractal basins of attraction, with attractor states being to (i) inflate forever, (ii) inflate over a short period of time then collapse, or (iii) collapse without inflating. Monerat et al. [122] investigated the dynamics of the pre-inflationary phase of the Universe and its exit to inflation in a closed FLRW model with radiation and a minimally coupled scalar field. They observe complex behavior associated with saddle-type critical points in phase space that give rise to deSitter attractors with multiple chaotic exits to inflation that depend on the structure of the scalar field potential. These results suggest that distinctions between exits to inflation may be manifested in the process of reheating and as a selected spectrum of inhomogeneous perturbations influenced by resonance mechanisms in curvature oscillations. This could possibly lead to fractal patterns in the density spectrum, gravitational waves, cosmic microwave background radiation (CMBR) field, or galaxy distribution that depend on specific details including the number of fields, the nature of the fields, and their interaction potentials.

(a)

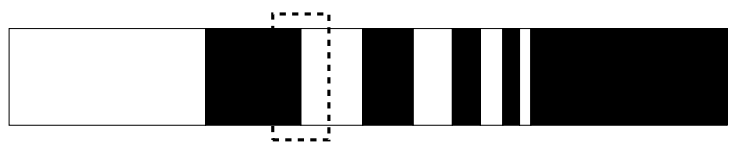

(b)

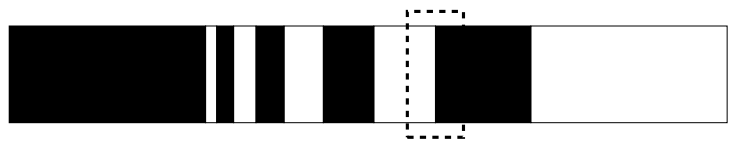

(c)

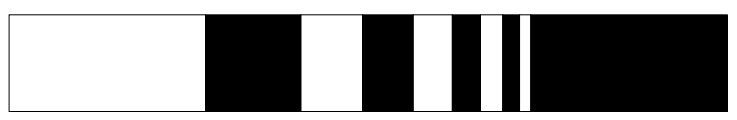

Figure 4: Fractal structure of the transition between reflected and captured states for colliding kinkantikink solitons in the parameter space of impact velocity for a $\lambda\left(\phi^{2}-1\right)^{2}$ scalar field potential. The top image (a) shows the 2-bounce windows in dark with the rightmost region $(v / c>0.25)$ representing the single-bounce regime above which no captured state exists, and the leftmost white region $(v / c<0.19)$ representing the captured state below which no reflection windows exist. Between these two marker velocities, there are 2-bounce reflection states of decreasing widths separated by regions of bion formation. Zooming in on the domain outlined by the dashed box, a self-similar structure is apparent in the middle image (b), where now the dark regions represent 3-bounce windows of decreasing widths. Zooming in once again on the boundaries of these 3-bounce windows, a similar structure is found as shown in the bottom image (c) but with 4-bounce reflection windows. This pattern of self-similarity characterized by $n$-bounce windows is observed at all scales investigated numerically.

Chaotic behavior can also be found in more general applications of scalar field dynamics. Anninos et al. [20] investigated the nonlinear behavior of colliding kink-antikink solitons or domain walls described by a single real scalar field with self-interaction potential $\lambda\left(\phi^{2}-1\right)^{2}$. Domain walls can form as topological defects during the spontaneous symmetry breaking period associated with phase transitions, and can seed density fluctuations in the large scale structure. For collisional time scales much smaller than the cosmological expansion, they find that whether a kink-antikink collision results in a bound state or a two-soliton solution depends on a fractal structure observed 
in the impact velocity parameter space. The fractal structure arises from a resonance condition associated with energy exchanges between translational modes and internal shape-mode oscillations. The impact parameter space is a complex self-similar fractal composed of sequences of different $n$-bounce (the number of bounces or oscillations in the transient semi-coherent state) reflection windows separated by regions of oscillating bion states (see Figure 4). They compute the Lyapunov exponents for parameters in which a bound state forms to demonstrate the chaotic nature of the bion oscillations.

\subsection{Quark-hadron phase transition}

The standard picture of cosmology assumes that a phase transition (associated with chiral symmetry breaking following the electroweak transition) occurred at approximately $10^{-5} \mathrm{~s}$ after the Big Bang to convert a plasma of free quarks and gluons into hadrons. Although this transition can be of significant cosmological importance, it is not known with certainty whether it is of first order or higher, and what the astrophysical consequences might be on the subsequent state of the Universe. For example, the transition may play a potentially observable role in the generation of primordial magnetic fields. The QCD transition may also give rise to important baryon number inhomogeneities which can affect the distribution of light element abundances from primordial Big Bang nucleosynthesis. The distribution of baryons may be influenced hydrodynamically by the competing effects of phase mixing and phase separation, which arise from any inherent instability of the interface surfaces separating regions of different phase. Unstable modes, if they exist, will distort phase boundaries and induce mixing and diffusive homogenization through hydrodynamic turbulence [102, 112, 95, 4, 137].

In an effort to support and expand theoretical studies, a number of one-dimensional numerical simulations have been carried out to explore the behavior of growing hadron bubbles and decaying quark droplets in simplified and isolated geometries. For example, Rezolla et al. [138] considered a first order phase transition and the nucleation of hadronic bubbles in a supercooled quarkgluon plasma, solving the relativistic Lagrangian equations for disconnected and evaporating quark regions during the final stages of the phase transition. They investigated numerically a single isolated quark drop with an initial radius large enough so that surface effects can be neglected. The droplet evolves as a self-similar solution until it evaporates to a sufficiently small radius that surface effects break the similarity solution and increase the evaporation rate. Their simulations indicate that, in neglecting long-range energy and momentum transfer (by electromagnetically interacting particles) and assuming that baryon number is transported with the hydrodynamical flux, the baryon number concentration is similar to what is predicted by chemical equilibrium calculations.

Kurki-Suonio and Laine [108] studied the growth of bubbles and the decay of droplets using a one-dimensional spherically symmetric code that accounts for a phenomenological model of the microscopic entropy generated at the phase transition surface. Incorporating the small scale effects of finite wall width and surface tension, but neglecting entropy and baryon flow through the droplet wall, they simulate the process by which nucleating bubbles grow and evolve to a similarity solution. They also compute the evaporation of quark droplets as they deviate from similarity solutions at late times due to surface tension and wall effects.

Ignatius et al. [96] carried out parameter studies of bubble growth for both the QCD and electroweak transitions in planar symmetry, demonstrating that hadron bubbles reach a stationary similarity state after a short time when bubbles grow at constant velocity. They investigated the stationary state using numerical and analytic methods, accounting also for preheating caused by shock fronts.

Fragile and Anninos [76] performed two-dimensional simulations of first order QCD transitions to explore the nature of interface boundaries beyond linear stability analysis, and determine if they

Living Reviews in Relativity

http://www. livingreviews.org/lrr-2001-2 

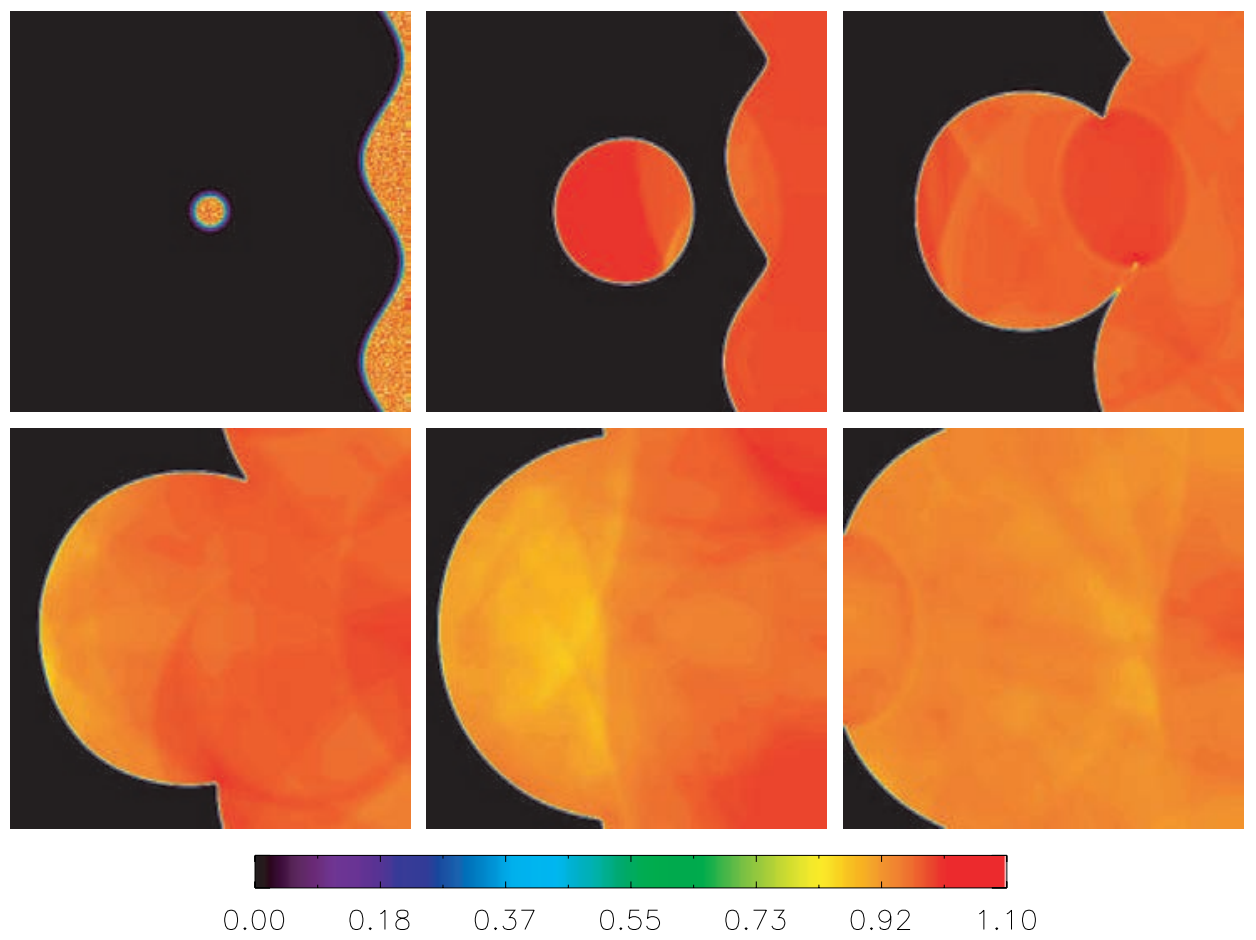

Figure 5: Image sequence of the scalar field from a $2 D$ calculation showing the interaction of two deflagration systems (one planar wall propagating from the right side, and one spherical bubble nucleating from the center). The physical size of the grid is set to $1000 \times 1000 \mathrm{fm}$ and resolved by $512 \times 512$ zones. The run time of the simulation is about two sound crossing times, where the sound speed is $c / \sqrt{3}$, so the shock fronts leading the condensing phase fronts travel across the grid twice. The hot quark (cold hadron) phases have smaller (larger) scalar field values and are represented by black (color) in the colormap. 

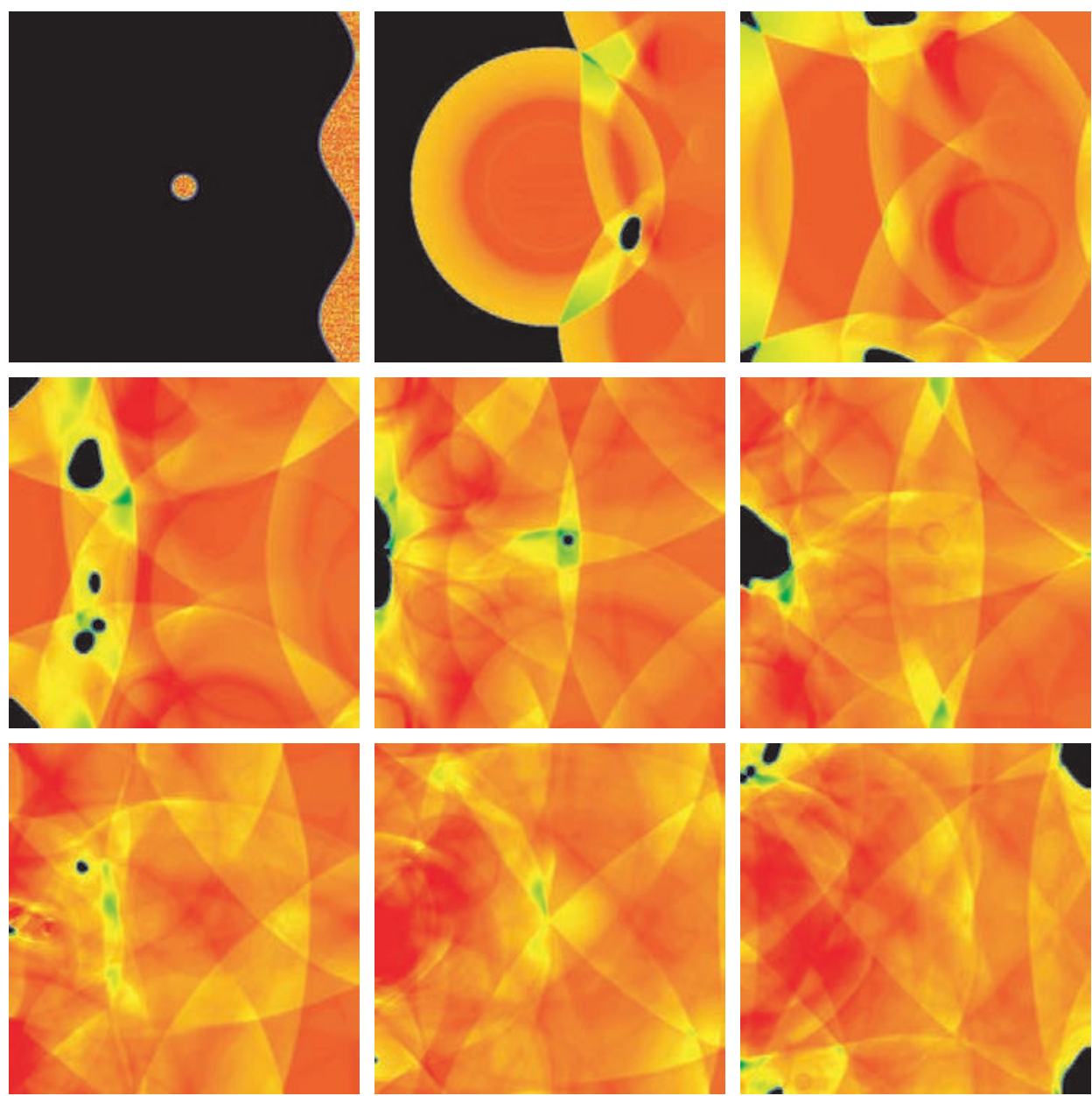

0.00

0.18

0.37

0.55

0.73

$0.92 \quad 1.10$

Figure 6: Image sequence of the scalar field from a $2 D$ calculation showing the interaction of two detonation systems (one planar wall propagating from the right side, and one spherical bubble nucleating from the center). The physical size of the grid is set to $1000 \times 1000 \mathrm{fm}$ and resolved by $1024 \times 1024$ zones. The run time of the simulation is about two sound crossing times. 

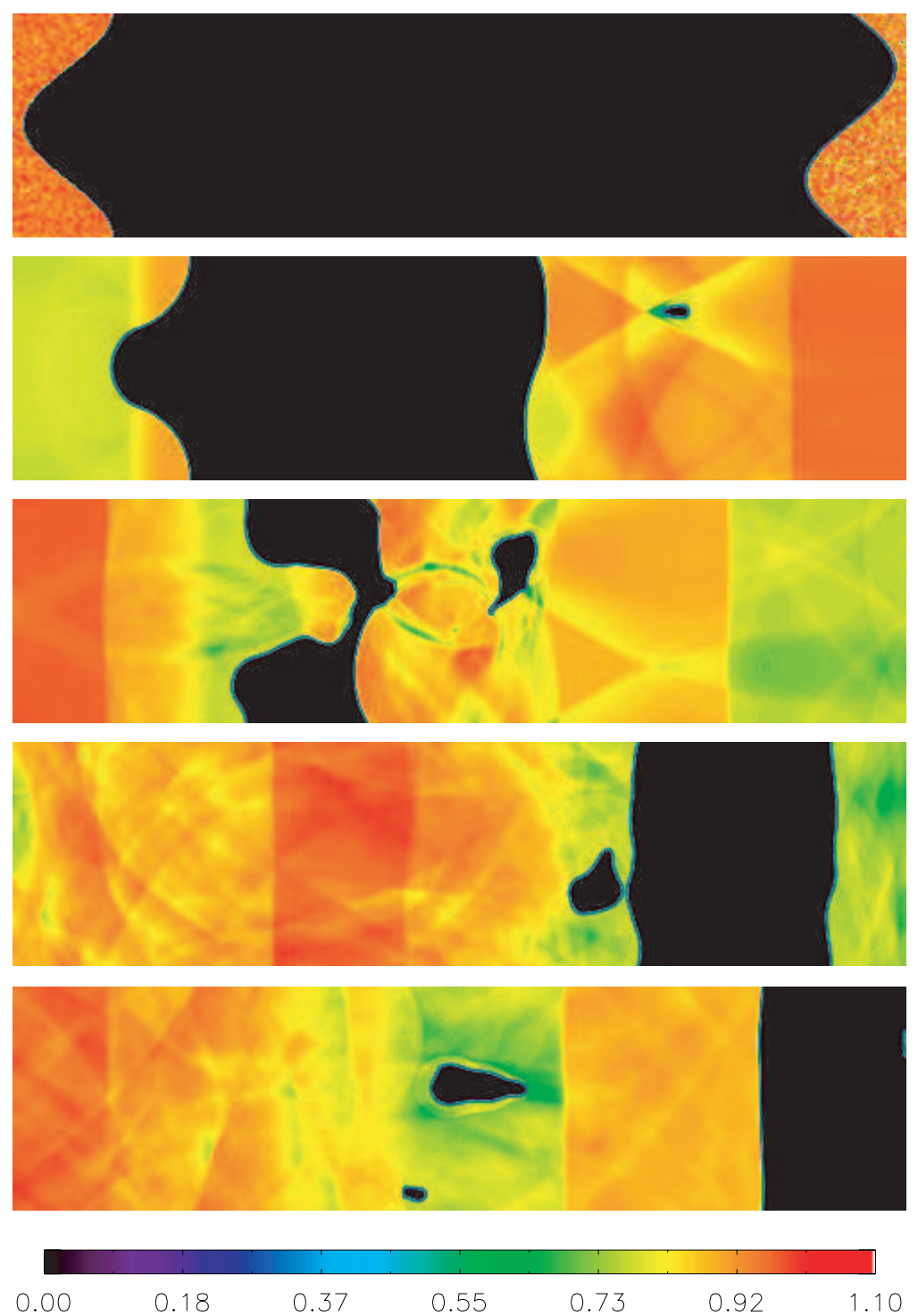

Figure 7: Image sequence of the scalar field from a 2D calculation showing the interaction of shock and rarefaction waves with a deflagration wall (initiated at the left side) and a detonation wall (starting from the right). A shock and rarefaction wave travel to the right and left, respectively, from the temperature discontinuity located initially at the grid center (the right half of the grid is at a higher temperature). The physical size of the domain is set to $1806.1 \times 451.53 \mathrm{fm}$ and resolved by $2048 \times 512$ zones. The run time of the simulation is about two sound crossing times. 
are stable when the full nonlinearities of the relativistic scalar field and hydrodynamic system of equations are accounted for. They used results from linear perturbation theory to define initial fluctuations on either side of the phase fronts and evolved the data numerically in time for both deflagration and detonation configurations. No evidence of mixing instabilities or hydrodynamic turbulence was found in any of the cases they considered, despite the fact that they investigated the parameter space predicted to be potentially unstable according to linear analysis. They also investigated whether phase mixing can occur through a turbulence-type mechanism triggered by shock proximity or disruption of phase fronts. They considered three basic cases (see image sequences in Figures 5, 6, and 7 below): interactions between planar and spherical deflagration bubbles, collisions between planar and spherical detonation bubbles, and a third case simulating the interaction between both deflagration and detonation systems initially at two different thermal states. Their results are consistent with the standard picture of cosmological phase transitions in which hadron bubbles expand as spherical condensation fronts, undergoing regular (non-turbulent) coalescence, and eventually leading to collapsing spherical quark droplets in a medium of hadrons. This is generally true even in the detonation cases which are complicated by greater entropy heating from shock interactions contributing to the irregular destruction of hadrons and the creation of quark nuggets.

However, Fragile and Anninos also note a deflagration 'instability' or acceleration mechanism evident in their third case for which they assume an initial thermal discontinuity in space separating different regions of nucleating hadron bubbles. The passage of a rarefaction wave (generated at the thermal discontinuity) through a slowly propagating deflagration can significantly accelerate the condensation process, suggesting that the dominant modes of condensation in an early Universe which super-cools at different rates within causally connected domains may be through supersonic detonations or fast moving (nearly sonic) deflagrations. A similar speculation was made by Kamionkowski and Freese [102] who suggested that deflagrations become unstable to perturbations and are converted to detonations by turbulent surface distortion effects. However, in the simulations, deflagrations are accelerated not from turbulent mixing and surface distortion, but from enhanced super-cooling by rarefaction waves. In multi-dimensions, the acceleration mechanism can be exaggerated further by upwind phase mergers due to transverse flow, surface distortion, and mode dissipation effects, a combination that may result in supersonic front propagation speeds, even if the nucleation process began as a slowly propagating deflagration.

\subsection{Nucleosynthesis}

Observations of the light elements produced during Big Bang nucleosynthesis following the quark/ hadron phase transition (roughly $10^{-2}-10^{2} \mathrm{~s}$ after the Big Bang) are in good agreement with the standard model of our Universe (see Section 2.2). However, it is interesting to investigate other more general models to assert the role of shear and curvature on the nucleosynthesis process, and place limits on deviations from the standard model.

Rothman and Matzner [140] considered primordial nucleosynthesis in anisotropic cosmologies, solving the strong reaction equations leading to ${ }^{4} \mathrm{He}$. They find that the concentration of ${ }^{4} \mathrm{He}$ increases with increasing shear due to time scale effects and the competition between dissipation and enhanced reaction rates from photon heating and neutrino blue shifts. Their results have been used to place a limit on anisotropy at the epoch of nucleosynthesis. Kurki-Suonio and Matzner [109] extended this work to include 30 strong 2-particle reactions involving nuclei with mass numbers $A \leq 7$, and to demonstrate the effects of anisotropy on the cosmologically significant isotopes ${ }^{2} \mathrm{H}$, ${ }^{3} \mathrm{He},{ }^{4} \mathrm{He}$ and ${ }^{7} \mathrm{Li}$ as a function of the baryon to photon ratio. They conclude that the effect of anisotropy on ${ }^{2} \mathrm{H}$ and ${ }^{3} \mathrm{He}$ is not significant, and the abundances of ${ }^{4} \mathrm{He}$ and ${ }^{7} \mathrm{Li}$ increase with anisotropy in accord with [140].

Furthermore, it is possible that neutron diffusion, the process whereby neutrons diffuse out from

Living Reviews in Relativity

http://www. livingreviews . org/lrr-2001-2 
regions of very high baryon density just before nucleosynthesis, can affect the neutron to proton ratio in such a way as to enhance deuterium and reduce ${ }^{4} \mathrm{He}$ compared to a homogeneous model. However, plane symmetric, general relativistic simulations with neutron diffusion [110] show that the neutrons diffuse back into the high density regions once nucleosynthesis begins there - thereby wiping out the effect. As a result, although inhomogeneities influence the element abundances, they do so at a much smaller degree then previously speculated. The numerical simulations also demonstrate that, because of the back diffusion, a cosmological model with a critical baryon density cannot be made consistent with helium and deuterium observations, even with substantial baryon inhomogeneities and the anticipated neutron diffusion effect.

\subsection{Cosmological gravitational waves}

Gravitational waves are an inevitable product of the Einstein equations, and one can expect a wide spectrum of wave signals propagating throughout our Universe due to anisotropic and inhomogeneous metric and matter fluctuations, collapsing matter structures, ringing black holes, and colliding neutron stars, for example. The discussion here is restricted to the pure vacuum field dynamics and the fundamental nonlinear behavior of gravitational waves in numerically generated cosmological spacetimes.

\subsubsection{Planar symmetry}

Centrella and Matzner [57, 58] studied a class of plane symmetric cosmologies representing gravitational inhomogeneities in the form of shocks or discontinuities separating two vacuum expanding Kasner cosmologies (2). By a suitable choice of parameters, the constraint equations can be satisfied at the initial time with a Euclidean 3-surface and an algebraic matching of parameters across the different Kasner regions that gives rise to a discontinuous extrinsic curvature tensor. They performed both numerical calculations and analytical estimates using a Green's function analysis to establish and verify (despite the numerical difficulties in evolving discontinuous data) certain aspects of the solutions, including gravitational wave interactions, the formation of tails, and the singularity behavior of colliding waves in expanding vacuum cosmologies.

Shortly thereafter, Centrella and Wilson [59,60] developed a polarized plane symmetric code for cosmology, adding also hydrodynamic sources with artificial viscosity methods for shock capturing and Barton's method for monotonic transport [162]. The evolutions are fully constrained (solving both the momentum and Hamiltonian constraints at each time step) and use the mean curvature slicing condition. This work was subsequently extended by Anninos et al. [9, 11, 7], implementing more robust numerical methods, an improved parametric treatment of the initial value problem, and generic unpolarized metrics.

In applications of these codes, Centrella [61] investigated nonlinear gravitational waves in Minkowski space and compared the full numerical solutions against a first order perturbation solution to benchmark certain numerical issues such as numerical damping and dispersion. A second order perturbation analysis was used to model the transition into the nonlinear regime. Anninos et al. [10] considered small and large perturbations in the two degenerate Kasner models: $p_{1}=p_{3}=0$ or $2 / 3$, and $p_{2}=1$ or $-1 / 3$, respectively, where $p_{i}$ are parameters in the Kasner metric (2). Carrying out a second order perturbation expansion and computing the Newman-Penrose (NP) scalars, Riemann invariants and Bel-Robinson vector, they demonstrated, for their particular class of spacetimes, that the nonlinear behavior is in the Coulomb (or background) part represented by the leading order term in the NP scalar $\Psi_{2}$, and not in the gravitational wave component. For standing-wave perturbations, the dominant second order effects in their variables are an enhanced monotonic increase in the background expansion rate, and the generation of oscillatory behavior in the background spacetime with frequencies equal to the harmonics of the first order standing-wave solution. 
Expanding these investigations of the Coulomb nonlinearity, Anninos and McKinney [16] used a gauge invariant perturbation formalism to construct constrained initial data for general relativistic cosmological sheets formed from the gravitational collapse of an ideal gas in a critically closed FLRW "background" model. They compared results to the Newtonian Zel'dovich [165] solution over a broad range of field strengths and flows, and showed that the enhanced growth rates of nonlinear modes (in both the gas density and Riemann curvature invariants) accelerate the collapse process significantly compared to Newtonian and perturbation theory. They also computed the back-reaction of these structures to the mean cosmological expansion rate and found only a small effect, even for cases with long wavelengths and large amplitudes. These structures were determined to produce time-dependent gravitational potential signatures in the CMBR (essentially fully relativistic Rees-Sciama effects) comparable to, but still dominated by, the large scale SachsWolfe anisotropies. This confirmed, and is consistent with, the assumptions built into Newtonian calculations of this effect.

\subsubsection{Multi-dimensional vacuum cosmologies}

Two additional examples of general relativistic codes developed for the purpose of investigating dynamical behaviors in non-flat, vacuum, cosmological topologies are attributed to Holcomb [91] and Ove [129]. Holcomb considered vacuum axisymmetric models to study the structure of General Relativity and the properties of gravitational waves in non-asymptotically flat spacetimes. The code was based on the ADM $3+1$ formalism and used Kasner matching conditions at the outer edges of the mesh, mean curvature slicing, and a shift vector to enforce the isothermal gauge in order to simplify the metric and to put it in a form that resembles quasi-isotropic coordinates. However, a numerical instability was observed in cases where the mesh domain exceeded the horizon size. This was attributed to the particular gauge chosen, which does not appear well-suited to the Kasner metric as it results in super-luminal coordinate velocities beyond the horizon scale.

Ove developed an independent code based on the ADM formalism to study cosmic censorship issues, including the nature of singular behavior allowed by the Einstein equations, the role of symmetry in the creation of singularities, the stability of Cauchy horizons, and whether black holes or a ring singularity can be formed by the collision of strong gravitational waves. Ove adopted periodic boundary conditions with 3-torus topology and a single Killing field, and therefore generalizes to two dimensions the planar codes discussed in the previous section. This code also used a variant of constant mean curvature slicing, was fully constrained at each time cycle, and the shift vector was chosen to put the metric into a (time-dependent) conformally flat form at each spatial hypersurface.

Living Reviews in Relativity

http: //www . livingreviews . org/Irr-2001-2 


\section{Physical Cosmology}

The phrase "physical cosmology" is generally associated with the large (galaxy and cluster) scale structure of the post-recombination epoch where gravitational effects are modeled approximately by Newtonian physics on an uniformly expanding, matter dominated FLRW background. A discussion of the large scale structure is included in this review since any viable model of our Universe which allows a regime where strongly general relativistic effects are important must match onto the weakly relativistic (or Newtonian) regime. Also, since certain aspects of this regime are directly observable, one can hope to constrain or rule out various cosmological models and/or parameters, including the density $\left(\Omega_{0}\right)$, Hubble $\left(H_{0}=100 h \mathrm{~km} \mathrm{~s}^{-1} \mathrm{Mpc}^{-1}\right)$, and cosmological $(\Lambda)$ constants.

Due to the vast body of literature on numerical simulations dealing with the post-recombination epoch, only a very small fraction of published work can be reviewed in this paper. Hence, the following summary is limited to cover just a few aspects of computational physical cosmology, and in particular those that can potentially be used to discriminate between cosmological model parameters, even within the realm of the standard model.

For a general overview of theoretical and observational issues associated with structure formation, the reader is referred to $[132,131]$, and to [45] for a broad review of numerical simulations (and methods) of structure formation.

\subsection{Cosmic microwave background}

The Cosmic Microwave Background Radiation (CMBR) is a direct relic of the early Universe, and currently provides the deepest probe of evolving cosmological structures. Although the CMBR is primarily a uniform black body spectrum throughout all space, fluctuations or anisotropies in the spectrum can be observed at very small levels to correlate with the matter density distribution. Comparisons between observations and simulations generally support the mostly isotropic, standard Big Bang model, and can be used to constrain the various proposed matter evolution scenarios and cosmological parameters. For example, sky survey satellite observations [34, 149] suggest a flat $\Lambda$-dominated Universe with scale-invariant Gaussian fluctuations that is consistent with numerical simulations of large sale structure formation (e.g., galaxy clusters, Ly $\alpha$ forest).

As shown in the timeline of Figure 8, CMBR signatures can be generally classified into two main components: primary and secondary anisotropies, separated by a Surface of Last Scattering (SoLS). Both of these components include contributions from two distinctive phases: a surface marking the threshold of decoupling of ions and electrons from hydrogen atoms in primary signals, and a surface of reionization marking the start of multiphase secondary contributions through nonlinear structure evolution, star formation, and radiative feedback from the small scales to the large.

\subsubsection{Primordial black body effects}

The black body spectrum of the isotropic background is essentially due to thermal equilibrium prior to the decoupling of ions and electrons, and few photon-matter interactions after that. At sufficiently high temperatures, prior to the decoupling epoch, matter was completely ionized into free protons, neutrons, and electrons. The CMB photons easily scatter off electrons, and frequent scattering produces a blackbody spectrum of photons through three main processes that occur faster than the Universe expands:

- Compton scattering in which photons transfer their momentum and energy to electrons if they have significant energy in the electron's rest frame. This is approximated by Thomson scattering if the photon's energy is much less than the rest mass. Inverse Compton scattering is also possible in which sufficiently energetic (relativistic) electrons can blueshift photons.

Living Reviews in Relativity

http: //www . livingreviews . org/lrr-2001-2 


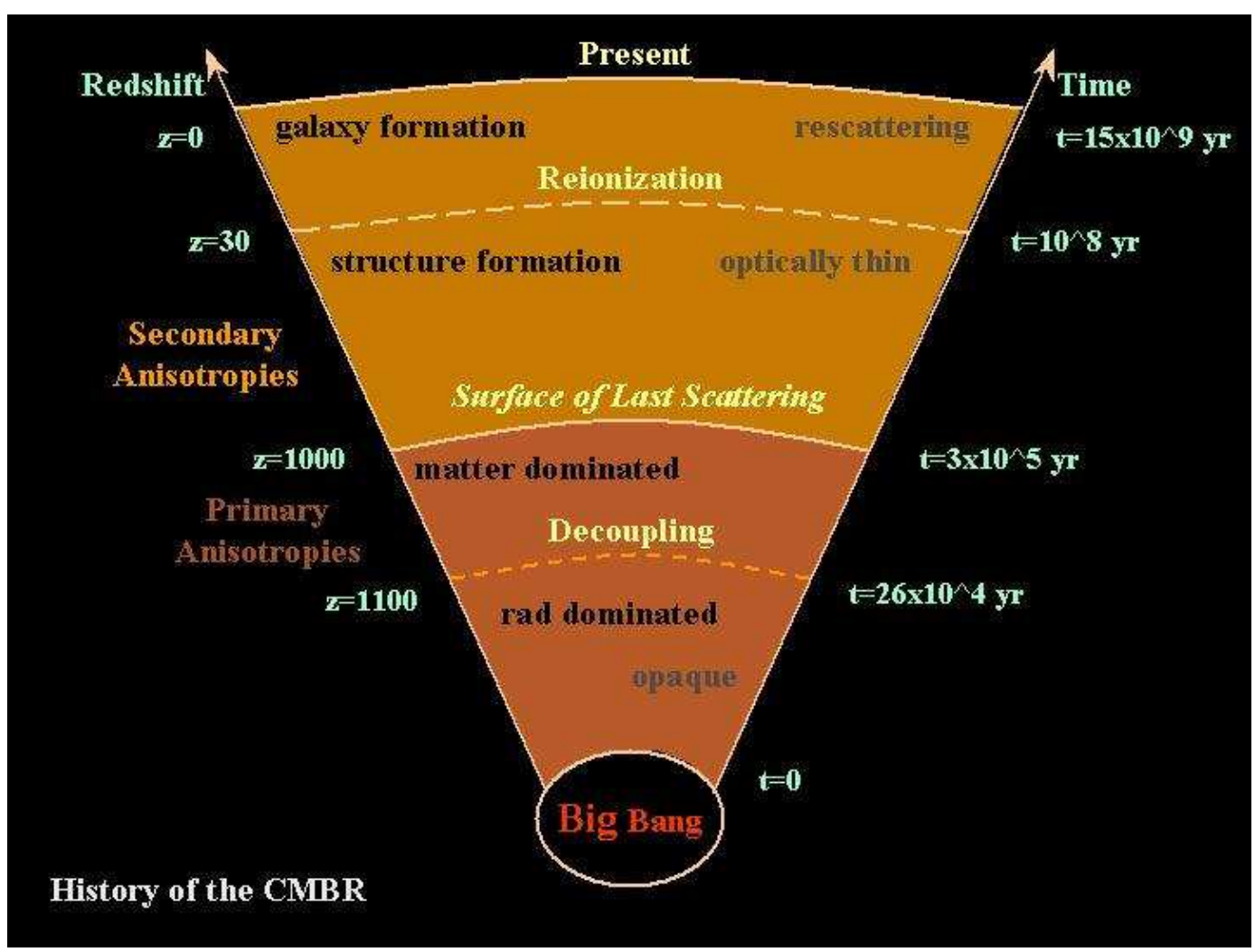

Figure 8: Historical time-line of the cosmic microwave background radiation showing the start of photon/nuclei combination, the surface of last scattering (SoLS), and the epoch of reionization due to early star formation. The times are represented in years (to the right) and redshift (to the left). Primary anisotropies are collectively attributed to the early effects at the last scattering surface and the large scale Sachs-Wolfe effect. Secondary anisotropies arise from path integration effects, reionization smearing, and higher order interactions with the evolving nonlinear structures at relatively low redshifts. 
- Double Compton scattering can both produce and absorb photons, and thus is able to thermalize photons to a Planck spectrum (unlike Compton scattering which conserves photon number, and, although it preserves a Planck spectrum, relaxes to a Bose-Einstein distribution).

- Bremsstrahlung emission of electromagnetic radiation due to the acceleration of electrons in the vicinity of ions. This also occurs in reverse (free-free absorption) since charged particles can absorb photons. In contrast to Coulomb scattering, which maintains thermal equilibrium among baryons without affecting photons, Bremsstrahlung tends to relax photons to a Planck distribution.

Although the CMBR is a unique and deep probe of both the thermal history of the early Universe and primordial perturbations in the matter distribution, the associated anisotropies are not exclusively primordial in nature. Important modifications to the CMBR spectrum, from both primary and secondary components, can arise from large scale coherent structures, even well after the photons decouple from the matter at redshift $z \sim 10^{3}$, due to gravitational redshifting, lensing, and scattering effects.

\subsubsection{Primary anisotropies}

The most important contributions to primary anisotropies between the start of decoupling and the surface of last scattering include the following effects:

- Sachs-Wolfe $(S W)$ effect: Gravitational redshift of photons between potentials at the SoLS and the present. It is the dominant effect at large angular scales comparable to the horizon size at decoupling $\left(\theta \sim 2^{\circ} \Omega^{1 / 2}\right)$.

- Doppler effect: Dipolar patterns are imprinted in the energy distribution from the peculiar velocities of the matter structures acting as the last scatterers of the photons.

- Acoustic peaks: Anisotropies at intermediate angular scales $\left(0.1^{\circ}<\theta<2^{\circ}\right)$ are atttributed to small scale processes occurring until decoupling, including acoustic oscillations of the baryonphoton fluid from primordial density inhomogeneities. This gives rise to acoustic peaks in the thermal spectrum representing the sound horizon scale at decoupling.

- SoLS damping: The electron capture rate is only slightly faster than photodissociation at the start of decoupling, causing the SoLS to have a finite thickness $(\Delta z \sim 100)$. Scattering over this interval damps fluctuations on scales smaller than the SoLS depth $\left(\theta<10^{\prime} \Omega^{1 / 2}\right)$.

- Silk damping: Photons can diffuse out of overdense regions, dragging baryons with them over small angular scales. This tends to suppress both density and radiation fluctuations.

All of these mechanisms perturb the black body background radiation since thermalization processes are not efficient at redshifts smaller than $\sim 10^{7}$.

\subsubsection{Secondary anisotropies}

Secondary anisotropies consist of two principal effects, gravitational and scattering. Some of the more important gravitational contributions to the CMB include:

- Early ISW effect: Photon contributions to the energy density of the Universe may be nonnegligible compared to ordinary matter (dark or baryonic) at the last scattering. The decreasing contribution of photons in time results in a decay of the potential, producing the early Integrated Sachs-Wolfe (ISW) effect. 
- Late ISW effect: In open cosmological models or models with a cosmological constant, the gravitational potential decays at late times due to a greater rate of expansion compared to flat spacetimes, producing the late ISW effect on large angular scales.

- Rees-Sciama effect: Evolving nonlinear strucutures (e.g., galaxies and clusters) generate time-varying potentials which can seed asymmetric energy shifts in photons crossing potential wells from the SoLS to the present.

- Lensing: In contrast to ISW effects which change the energy but not directions of the photons, gravitational lensing deflects the paths without changing the energy. This effectively smears out the imaging of the SoLS.

- Proper motion: Compact objects such as galaxy clusters can imprint a dipolar pattern in the $\mathrm{CMB}$ as they move across the sky.

- Gravitational waves: Perturbations in the spacetime fabric affect photon paths, energies, and polarizations, predominantly at scales larger than the horizon at decoupling.

Secondary scattering effects are associated with reionization and their significance depends on when and over what scales it takes place. Early reionization leads to large optical depths and greater damping due to secondary scattering. Over large scales, reionization has little effect since these scales are not in causal contact. At small scales, primordial anisotropies can be wiped out entirely and replaced by secondary ones. Some of the more important secondary scattering effects include:

- Thomson scattering: Photons are scattered by free electrons at sufficiently large optical depths achieved when the Universe undergoes a global reionization at late times. This damps out fluctuations since energies are averaged over different directions in space.

- Vishniac effect: In a reionized Universe, high order coupling between the bulk flow of electrons and their density fluctuations generates new anisotropies at small angles.

- Thermal Sunyaev-Zel'dovich effect: Inverse Compton scattering of the CMB by hot electrons in the intracluster gas of a cluster of galaxies distorts the black body spectrum of the CMB. Low frequency photons will be shifted to high frequencies.

- Kinetic Sunyaev-Zel'dovich effect: The peculiar velocities of clusters produces anisotropies via a Doppler effect to shift the temperature without distorting the spectral form. Its effect is proportional to the product of velocity and optical depth.

- Polarization: Scattering of anisotropic radiation affects polarization due to the angular dependence of scattering. Polarization in turn affects anisotropies through a similar dependency and tends to damp anisotropies.

To make meaningful comparisons between numerical models and observed data, all of these (low and high order) effects from both the primary and secondary contributions (see for example Section 4.1 .4 and $[94,101]$ ) must be incorporated self-consistently into any numerical model, and to high accuracy in order to resolve and distinguish amongst the various weak signals. The following sections describe some work focused on incorporating many of these effects into a variety of largescale numerical cosmological models.

Living Reviews in Relativity

http: //www. livingreviews. org/lrr-2001-2 


\subsubsection{Computing CMBR anisotropies with ray-tracing methods}

Many efforts based on linear perturbation theory have been carried out to estimate temperature anisotropies in our Universe (for example see [114] and references cited in [131, 94]). Although such linearized approaches yield reasonable results, they are not well-suited to discussing the expected imaging of the developing nonlinear structures in the microwave background. Also, because photons are intrinsically coupled to the baryon and dark matter thermal and gravitational states at all spatial scales, a fully self-consistent treatment is needed to accurately resolve the more subtle features of the CMBR. This can be achieved with a ray-tracing approach based on MonteCarlo methods to track individual photons and their interactions through the evolving matter distributions. A fairly complete simulation involves solving the geodesic equations of motion for the collisionless dark matter which dominate potential interactions, the hydrodynamic equations for baryonic matter with high Mach number shock capturing capability, the transport equations for photon trajectories, a reionization model to reheat the Universe at late times, the chemical kinetics equations for the ion and electron concentrations of the dominant hydrogen and helium gases, and the photon-matter interaction terms describing scattering, redshifting, depletion, lensing, and Doppler effects.

Such an approach has been developed by Anninos et al. [15], and applied to a Hot Dark Matter (HDM) model of structure formation. In order to match both the observed galaxy-galaxy correlation function and COBE measurements of the CMBR, they find, for that model and neglecting reionization, the cosmological parameters are severely constrained to $\Omega_{0} h^{2} \approx 1$, where $\Omega_{0}$ and $h$ are the density and Hubble parameters respectively.

In models where the IGM does not reionize, the probability of scattering after the photonmatter decoupling epoch is low, and the Sachs-Wolfe effect dominates the anisotropies at angular scales larger than a few degrees. However, if reionization occurs, the scattering probability increases substantially and the matter structures, which develop large bulk motions relative to the comoving background, induce Doppler shifts on the scattered CMBR photons and leave an imprint of the surface of last scattering. The induced fluctuations on subhorizon scales in reionization scenarios can be a significant fraction of the primordial anisotropies, as observed by Tuluie et al. [157] also using ray-tracing methods. They considered two possible scenarios of reionization: A model that suffers early and gradual (EG) reionization of the IGM as caused by the photoionizing UV radiation emitted by decaying neutrinos, and the late and sudden (LS) scenario as might be applicable to the case of an early generation of star formation activity at high redshifts. Considering the HDM model with $\Omega_{0}=1$ and $h=0.55$, which produces CMBR anisotropies above current COBE limits when no reionization is included (see Section 4.1.4), they find that the EG scenario effectively reduces the anisotropies to the levels observed by COBE and generates smaller Doppler shift anisotropies than the LS model, as demonstrated in Figure 9. The LS scenario of reionization is not able to reduce the anisotropy levels below the COBE limits, and can even give rise to greater Doppler shifts than expected at decoupling.

Additional sources of CMBR anisotropy can arise from the interactions of photons with dynamically evolving matter structures and nonstatic gravitational potentials. Tuluie et al. [156] considered the impact of nonlinear matter condensations on the CMBR in $\Omega_{0} \leq 1$ Cold Dark Matter (CDM) models, focusing on the relative importance of secondary temperature anisotropies due to three different effects: (i) time-dependent variations in the gravitational potential of nonlinear structures as a result of collapse or expansion (the Rees-Sciama effect), (ii) proper motion of nonlinear structures such as clusters and superclusters across the sky, and (iii) the decaying gravitational potential effect from the evolution of perturbations in open models. They applied the ray-tracing procedure of [15] to explore the relative importance of these secondary anisotropies as a function of the density parameter $\Omega_{0}$ and the scale of matter distributions. They find that secondary temperature anisotropies are dominated by the decaying potential effect at large scales,

Living Reviews in Relativity

http: //www . livingreviews . org/lrr-2001-2 

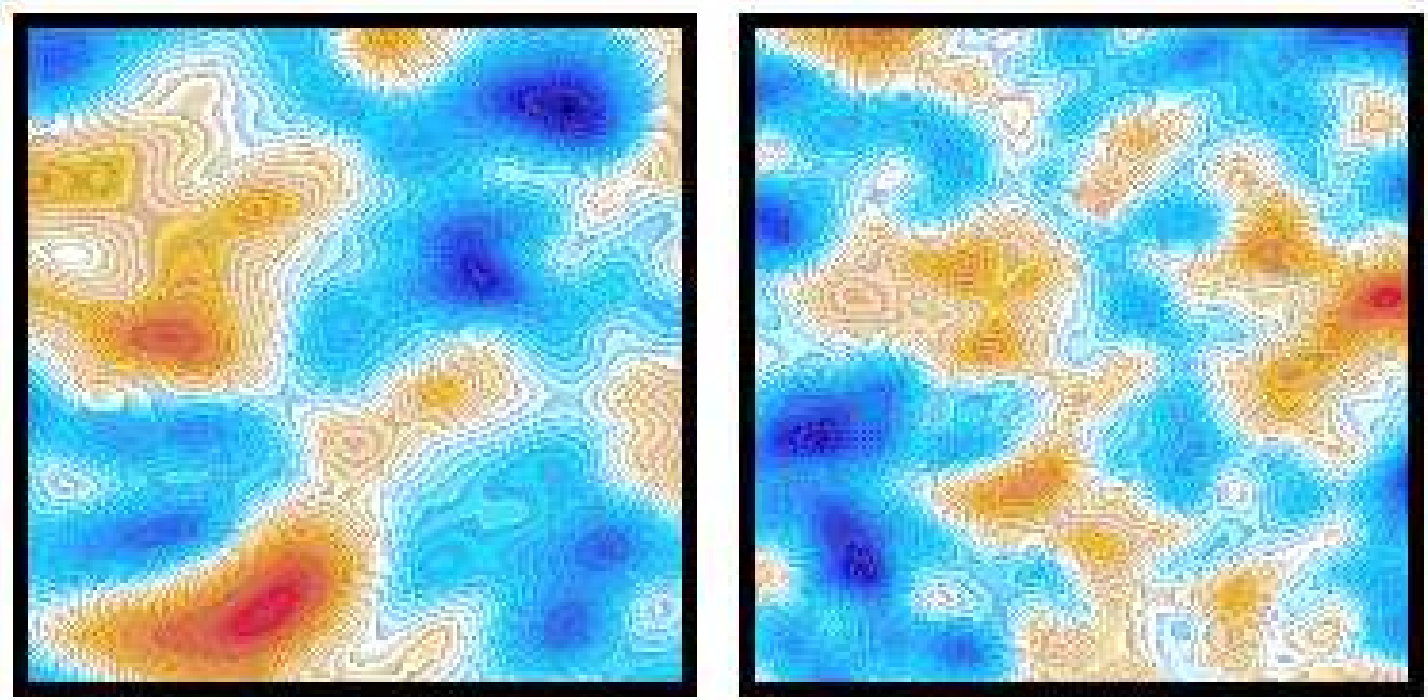

$\Gamma 1 \ldots 1$ m I

Г1แI н $?$
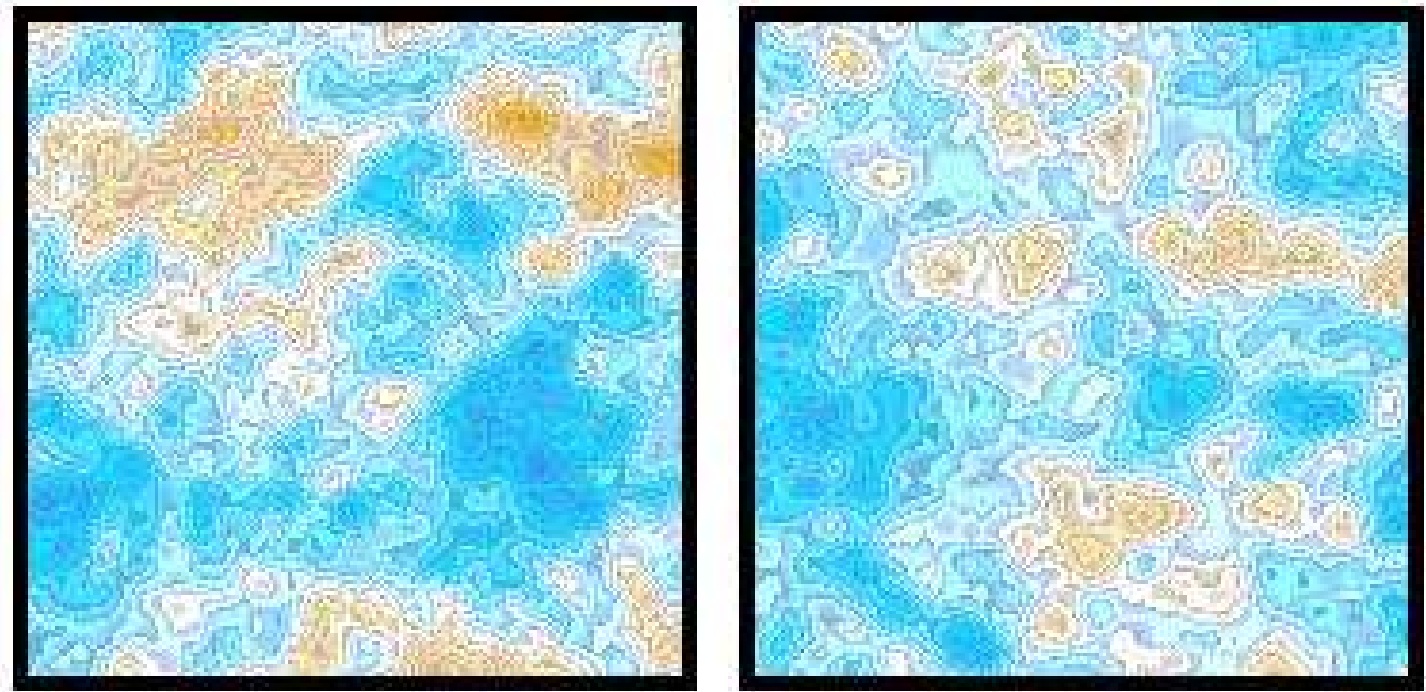

HJatc a

Hute I

Figure 9: Temperature fluctuations $(\Delta T / T)$ in the CMBR due to the primary Sachs-Wolfe (SW) effect and secondary integrated $S W$, Doppler, and Thomson scattering effects in a critically closed model. The top two plates are results with no reionization and baryon fractions 0.02 (plate $1,4^{\circ} \times 4^{\circ}$, $\left.\Delta T /\left.T\right|_{\mathrm{rms}}=2.8 \times 10^{-5}\right)$, and $0.2\left(\right.$ plate $\left.2,8^{\circ} \times 8^{\circ}, \Delta T /\left.T\right|_{\mathrm{rms}}=3.4 \times 10^{-5}\right)$. The bottom two plates are results from an "early and gradual" reionization scenario of decaying neutrinos with baryon fraction 0.02 (plate $3,4^{\circ} \times 4^{\circ}, \Delta T /\left.T\right|_{\mathrm{rms}}=1.3 \times 10^{-5}$; and plate $4,8^{\circ} \times 8^{\circ}, \Delta T /\left.T\right|_{\mathrm{rms}}=1.4 \times 10^{-5}$ ). If reionization occurs, the scattering probability increases and anisotropies are damped with each scattering event. At the same time, matter structures develop large bulk motions relative to the comoving background and induce Doppler shifts on the CMB. The imprint of this effect from last scattering can be a significant fraction of primary anisotropies.

Living Reviews in Relativity

http://www. livingreviews. org/lrr-2001-2 
but that all three sources of anisotropy can produce signatures of order $\Delta T /\left.T\right|_{\mathrm{rms}} \sim 10^{-6}$ as shown in Figure 10.

In addition to the effects discussed in this section, many other sources of secondary anisotropies (as mentioned in Section 4.1, including gravitational lensing, the Vishniac effect accounting for matter velocities and flows into local potential wells, and the Sunyaev-Zel'dovich (SZ) (Section 4.5.4) distortions from the Compton scattering of CMB photons by electrons in the hot cluster medium) can also be fairly significant. See [94, 152, 28, 80, 93] for more thorough discussions of the different sources of CMBR anisotropies.

\subsection{Gravitational lensing}

Observations of gravitational lenses [143] provide measures of the abundance and strength of nonlinear potential fluctuations along the lines of sight to distant objects. Since these calculations are sensitive to the gravitational potential, they may be more reliable than galaxy and velocity field measurements as they are not subject to the same ambiguities associated with biasing effects. Also, because different cosmological models predict different mass distributions, especially at the higher redshifts, lensing calculations can potentially be used to confirm or discard competing cosmological models.

As an alternative to solving the computationally demanding lens equations, Cen et al. [55] developed an efficient scheme to identify regions with surface densities capable of generating multiple images accurately for splittings larger than three arcseconds. They applied this technique to a standard CDM model with $\Omega_{0}=1$, and found that this model predicts more large angle splittings $\left(>8^{\prime \prime}\right)$ than are known to exist in the observed Universe. Their results suggest that the standard CDM model should be excluded as a viable model of our Universe. A similar analysis for a flat low density CDM model with a cosmological constant $\left(\Omega_{0}=0.3, \Lambda / 3 H_{0}^{2}=0.7\right)$ suggests a lower and perhaps acceptable number of lensing events. However, an uncertainty in their studies is the nature of the lenses at and below the resolution of the numerical grid. They model the lensing structures as simplified Singular Isothermal Spheres (SIS) with no distinctive cores.

Large angle splittings are generally attributed to larger structures such as clusters of galaxies, and there are indications that clusters have small but finite core radii $r_{\text {core }} \sim 20-30 h^{-1} \mathrm{kpc}$. Core radii of this size can have an important effect on the probability of multiple imaging. Flores and Primack [74] considered the effects of assuming two different kinds of splitting sources: isothermal spheres with small but finite core radii and radial density profiles $\rho \propto\left(r^{2}+r_{\text {core }}^{2}\right)^{-1}$, and spheres with a Hernquist density profile $\rho \propto r^{-1}(r+a)^{-3}$, where $r_{\text {core }} \sim 20-30 h^{-1} \mathrm{kpc}$ and $a \sim 300 h^{-1} \mathrm{kpc}$. They find that the computed frequency of large-angle splittings, when using the nonsingular profiles, can potentially decrease by more than an order of magnitude relative to the SIS case and can bring the standard CDM model into better agreement with observations. They stress that lensing events are sensitive to both the cosmological model (essentially the number density of lenses) and to the inner lens structure, making it difficult to probe the models until the structure of the lenses, both observationally and numerically, is better known.

\subsection{First star formation}

In CDM cosmogonies, the fluctuation spectrum at small wavelengths has a logarithmic dependence at mass scales smaller than $10^{8}$ solar masses, which indicates that all small scale fluctuations in this model collapse nearly simultaneously in time. This leads to very complex dynamics during the formation of these first structures. Furthermore, the cooling in these fluctuations is dominated by the rotational/vibrational modes of hydrogen molecules that were able to form using the free electrons left over from recombination and those produced by strong shock waves as catalysts. The first structures to collapse may be capable of producing pop III stars and have a substantial 


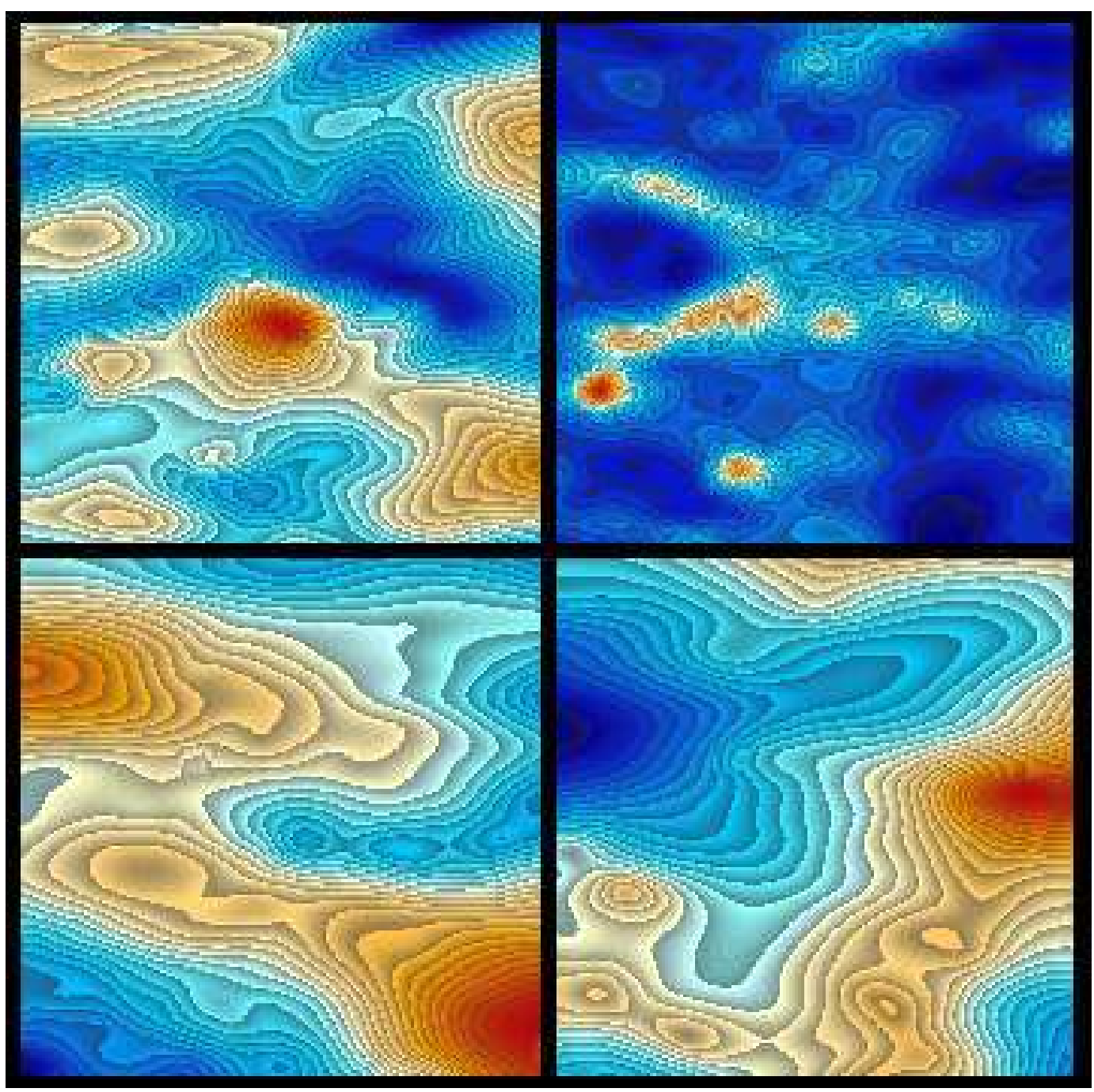

Figure 10: Secondary anisotropies from the proper motion of galaxy clusters across the sky and Rees-Sciama effects are presented in the upper-left image over $8^{\circ} \times 8^{\circ}$ in a critically closed Cold Dark Matter model. The corresponding column density of matter over the same region $(z=0.43$, $\Delta z=0.025)$ is displayed in the upper-right, clearly showing the dipolar nature of the proper motion effect. Anisotropies arising from decaying potentials in an open $\Omega=0.3$ model over a scale of $8^{\circ} \times 8^{\circ}$ are shown in the bottom left image, along with the gravitational potential over the same region $(z=0.33, \Delta z=0.03)$ in the bottom right, demonstrating a clear anti-correlation. Maximum temperature fluctuations in each simulation are $\Delta T / T=\left(5 \times 10^{-7}, 1.0 \times 10^{-6}\right)$ respectvely. Secondary anisotropies are dominated by decaying potentials at large scales, but all three sources (decaying potential, proper motion, and $R$-S) produce signatures of order $10^{-6}$.

Living Reviews in Relativity

http: //www. livingreviews. org/lrr-2001-2 
influence on the subsequent thermal evolution of the intergalactic medium, as suggested by Figure 2, due to the radiation emitted by the first generation stars as well as supernova driven winds. To know the subsequent fate of the Universe and which structures will survive or be destroyed by the UV background, it is first necessary to know when and how the first stars formed.

Ostriker and Gnedin [127] have carried out high resolution numerical simulations of the reheating and reionization of the Universe due to star formation bursts triggered by molecular hydrogen cooling. Accounting for the chemistry of the primeval hydrogen/helium plasma, self-shielding of the gas, radiative cooling, and a phenomenological model of star formation, they find that two distinct star populations form: the first generation pop III from $\mathrm{H}_{2}$ cooling prior to reheating at redshift $z \geq 14$; and the second generation pop II at $z<10$ when the virial temperature of the gas clumps reaches $10^{4} \mathrm{~K}$ and hydrogen line cooling becomes efficient. Star formation slows in the intermittent epoch due to the depletion of $H_{2}$ by photo-destruction and reheating. In addition, the objects which formed pop III stars also initiate pop II sequences when their virial temperatures reach $10^{4} \mathrm{~K}$ through continued mass accretion.

In resolving the details of a single star forming region in a CDM Universe, Abel et al. [2, 3] implemented a non-equilibrium radiative cooling and chemistry model $[1,21]$ together with the hydrodynamics and dark matter equations, evolving nine separate atomic and molecular species $\left(\mathrm{H}, \mathrm{H}^{+}, \mathrm{He}, \mathrm{He}^{+}, \mathrm{He}^{++}, \mathrm{H}^{-}, \mathrm{H}_{2}^{+}, \mathrm{H}_{2}\right.$, and $\mathrm{e}^{-}$, according to the reactive network described in Section 6.4.1) on nested and adaptively refined numerical grids. They follow the collapse and fragmentation of primordial clouds over many decades in mass and spatial dynamical range, finding a core of mass $\sim 200 M_{\odot}$ forms from a halo of about $\sim 10^{5} M_{\odot}$ (where a significant number fraction of hydrogen molecules are created) after less than one percent of the halo gas cools by molecular line emission. Bromm et al. [48] use a different Smoothed Particle Hydrodynamics (SPH) technique and a six species model $\left(\mathrm{H}, \mathrm{H}^{+}, \mathrm{H}^{-}, \mathrm{H}_{2}^{+}, \mathrm{H}_{2}\right.$, and $\left.\mathrm{e}^{-}\right)$to investigate the initial mass function of the first generation pop III stars. They evolve an isolated $3 \sigma$ peak of mass $2 \times 10^{6} M_{\odot}$ which collapses at redshift $z \sim 30$ and forms clumps of mass $10^{2}-10^{3} M_{\odot}$ which then grow by accretion and merging, suggesting that the very first stars were massive and in agreement with [3].

The implications of an early era of massive star populations on the thermal and chemical state of the intergalactic medium was investigated by Yoshida et al. [164]. They considered the effects of feedback and radiation transfer in early structure formation simulations to show that a significant fraction of the IGM can be ionized and polluted by metals from the first stars to form and become supernovae by $z \sim 15$, thus affecting subsequent stellar populations. They also argue that observed elemental abundances in the intracluster medium are not affected by metals originating from the first stars.

\subsection{Ly $\alpha$ forest}

The Ly $\alpha$ forest represents the optically thin (at the Lyman edge) component of Quasar Absorption Systems (QAS), a collection of absorption features in quasar spectra extending back to high redshifts. QAS are effective probes of the matter distribution and the physical state of the Universe at early epochs when structures such as galaxies are still forming and evolving. The relative lack of constraining observational data at the intermediate to high redshifts $(0<z<5)$, where differences between competing cosmological models are more pronounced, suggests that QAS can potentially yield valuable and discriminating observational data.

Many complex multi-component numerical simulations have been performed of the Lyman forest, which include the effects of dark matter (N-body), baryons (hydrodynamics), chemical composition (reactive networks), and microphysical response (radiative cooling and heating). See, for example, $[67,118,166]$, which represent some of the earliest comprehensive simulations. For the most part, all these calculations have been able to fit the observations reasonably well, including the column density and Doppler width distributions, the size of absorbers [62], and the line number 
evolution. Despite the fact that the cosmological models and parameters are different in each case, the simulations give roughly similar results provided that the proper ionization bias is used, $b_{\text {ion }} \equiv\left(\Omega_{\mathrm{b}} h^{2}\right)^{2} / \Gamma$, where $\Omega_{\mathrm{b}}$ is the baryonic density parameter, $h$ is the Hubble parameter and $\Gamma$ is the photoionization rate at the hydrogen Lyman edge. (However, see [50] for a discussion of the sensitivity of statistical properties on numerical resolution.) A theoretical paradigm has thus emerged from these calculations in which Ly $\alpha$ absorption lines originate from the relatively smaller scale structure in pregalactic or intergalactic gas through the bottom-up hierarchical formation picture in CDM-like Universes. The absorption features originate in structures exhibiting a variety of morphologies commonly found in numerical simulations (see Figure 11), including fluctuations in underdense regions, spheroidal minihalos, and filaments extending over scales of a few Mpc.

Machacek et al. [115] expanded on earlier work to compare several Ly $\alpha$ statistical measures from five different background cosmological models, including standard critical density Cold Dark Matter (CDM), open CDM, flat CDM with a cosmological constant, standard CDM with a tilted density spectrum, and a flat model with mixed hot and cold dark matter. All models were chosen to match local or low redshift observations, and most were also consistent with COBE measurements of the CMBR. The calculations were designed to establish which statistics are sensitive to different cosmological models. In particular, they find that the line number count above a given column density threshold is relatively insensitive to background models. On the other hand, the shape of the optical depth probability distribution function is strongly correlated to the amount of small scale power in density fluctuations, and is thus a good discriminator among models on scales of a few hundred kpc.

Meiksin et al. [117] followed up with more detailed comparisons of Ly $\alpha$ systems in several cosmologies with observed high resolution QSO spectra. Although all models are consistent with previous studies in that they give reasonably good statistical agreement with observed Ly $\alpha$ properties, under closer scrutiny none of the numerical models they considered passed all the tests, which included spectral flux, wavelet decomposed amplitude, and absorption line profile distributions. They suggest that comparisons might be improved, particularly in optically thin systems, by more energy injection into the IGM from late $\mathrm{He}^{+}$reionization or supernovae-driven winds, or by a larger baryon fraction.

\subsection{Galaxy clusters}

Clusters of galaxies are the largest gravitationally bound systems known to be in quasi-equilibrium. This allows for reliable estimates to be made of their mass as well as their dynamical and thermal attributes. The richest clusters, arising from $3 \sigma$ density fluctuations, can be as massive as $10^{14}-10^{15} M_{\odot}$, and the environment in these structures is composed of shock heated gas with temperatures of order $10^{7} \mathrm{mbox}--10^{8} \mathrm{~K}$ which emits thermal bremsstrahlung and line radiation at X-ray energies. Also, because of their spatial size of $\sim 1 h^{-1} \mathrm{Mpc}$ and separations of order $50 h^{-1} \mathrm{Mpc}$, they provide a measure of nonlinearity on scales close to the perturbation normalization scale $8 h^{-1} \mathrm{Mpc}$. Observations of the substructure, distribution, luminosity, and evolution of galaxy clusters are therefore likely to provide signatures of the underlying cosmology of our Universe, and can be used as cosmological probes in the observable redshift range $0 \leq z \leq 1$.

\subsubsection{Internal structure}

Thomas et al. [155] investigated the internal structure of galaxy clusters formed in high resolution N-body simulations of four different cosmological models, including standard, open, and flat but low density Universes. They find that the structure of relaxed clusters is similar in the critical and low density Universes, although the critical density models contain relatively more disordered clusters due to the freeze-out of fluctuations in open Universes at late times. The profiles of relaxed

Living Reviews in Relativity

http://www. livingreviews . org//rr-2001-2 


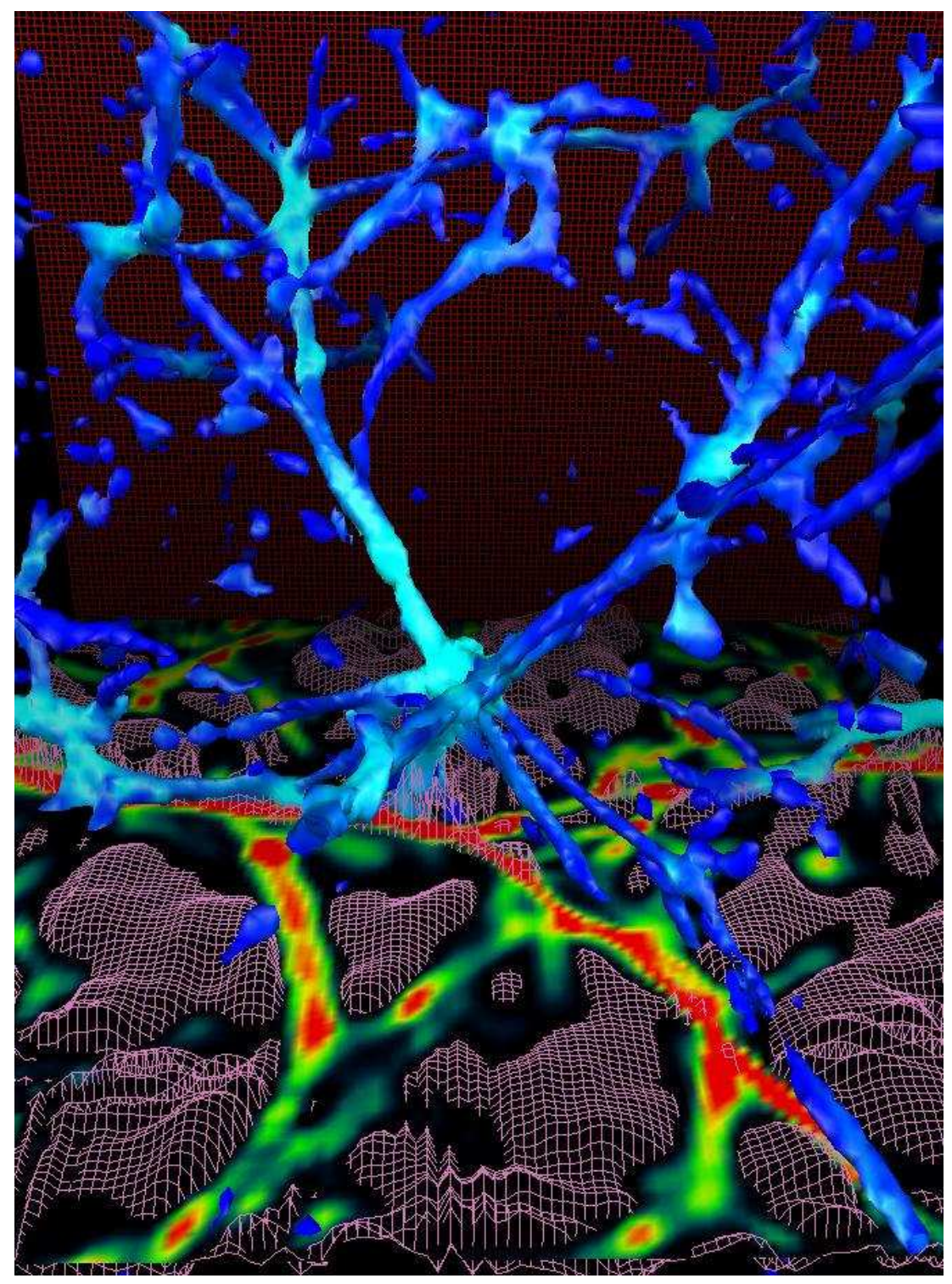

Figure 11: Distribution of the gas density at redshift $z=3$ from a numerical hydrodynamics

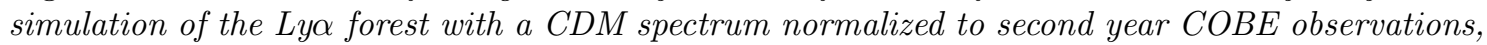
Hubble parameter of $h=0.5$, a comoving box size of $9.6 \mathrm{Mpc}$, and baryonic density of $\Omega_{\mathrm{b}}=0.06$ composed of $76 \%$ hydrogen and $24 \%$ helium. The region shown is 2.4 Mpc (proper) on a side. The isosurfaces represent baryons at ten times the mean density and are color coded to the gas temperature (dark blue $=3 \times 10^{4} \mathrm{~K}$, light blue $\left.=3 \times 10^{5} \mathrm{~K}\right)$. The higher density contours trace out isolated spherical structures typically found at the intersections of the filaments. A single random slice through the cube is also shown, with the baryonic overdensity represented by a rainbow-like color map changing from black (minimum) to red (maximum). The $\mathrm{He}^{+}$mass fraction is shown with a wire mesh in this same slice. To emphasize fine structure in the minivoids, the mass fraction in the overdense regions has been rescaled by the gas overdensity wherever it exceeds unity. 
clusters are very similar in the different simulations since most clusters are in a quasi-equilibrium state inside the virial radius and generally follow the universal density profile of Navarro et al. [125]. There does not appear to be a strong cosmological dependence in the profiles as suggested by previous studies of clusters formed from pure power law initial density fluctuations [65]. However, because more young and dynamically evolving clusters are found in critical density Universes, Thomas et al. suggest that it may be possible to discriminate among the density parameters by looking for multiple cores in the substructure of the dynamic cluster population. They note that a statistical population of 20 clusters could distinguish between open and critically closed Universes.

\subsubsection{Number density evolution}

The evolution of the number density of rich clusters of galaxies can be used to compute $\Omega_{0}$ and $\sigma_{8}$ (the power spectrum normalization on scales of $8 h^{-1} \mathrm{Mpc}$ ) when numerical simulation results are combined with the constraint $\sigma_{8} \Omega_{0}^{0.5} \approx 0.5$, derived from observed present-day abundances of rich clusters. Bahcall et al. [24] computed the evolution of the cluster mass function in five different cosmological model simulations and find that the number of high mass (Coma-like) clusters in flat, low $\sigma_{8}$ models (i.e., the standard CDM model with $\sigma_{8} \approx 0.5$ ) decreases dramatically by a factor of approximately $10^{3}$ from $z=0$ to $z \approx 0.5$. For low $\Omega_{0}$, high $\sigma_{8}$ models, the data result in a much slower decrease in the number density of clusters over the same redshift interval. Comparing these results to observations of rich clusters in the real Universe, which indicate only a slight evolution of cluster abundances to redshifts $z \approx 0.5-1$, they conclude that critically closed standard CDM and Mixed Dark Matter (MDM) models are not consistent with the observed data. The models which best fit the data are the open models with low bias $\left(\Omega_{0}=0.3 \pm 0.1\right.$ and $\left.\sigma_{8}=0.85 \pm 0.5\right)$, and flat low density models with a cosmological constant $\left(\Omega_{0}=0.34 \pm 0.13\right.$ and $\left.\Omega_{0}+\Lambda=1\right)$.

\subsubsection{X-ray luminosity function}

The evolution of the X-ray luminosity function, as well as the number, size and temperature distribution of galaxy clusters are all potentially important discriminants of cosmological models and the underlying initial density power spectrum that gives rise to these structures. Because the Xray luminosity (principally due to thermal bremsstrahlung emission from electron/ion interactions in the hot fully ionized cluster medium) is proportional to the square of the gas density, the contrast between cluster and background intensities is large enough to provide a window of observations that is especially sensitive to cluster structure. Comparisons of simulated and observed X-ray functions may be used to deduce the amplitude and shape of the fluctuation spectrum, the mean density of the Universe, the mass fraction of baryons, the structure formation model, and the background cosmological model.

Several groups $[49,56]$ have examined the properties of X-ray clusters in high resolution numerical simulations of a standard CDM model normalized to COBE. Although the results are very sensitive to grid resolution (see [17] for a discussion of the effects from resolution constraints on the properties of rich clusters), their primary conclusion, that the standard CDM model predicts too many bright X-ray emitting clusters and too much integrated X-ray intensity, is robust since an increase in resolution will only exaggerate these problems. On the other hand, similar calculations with different cosmological models [56, 52] suggest reasonable agreement of observed data with Cold Dark Matter + cosmological constant $(\Lambda \mathrm{CDM})$, Cold + Hot Dark Matter $(\mathrm{CHDM})$, and Open or low density CDM (OCDM) evolutions due to different universal expansions and density power spectra.

Living Reviews in Relativity

http://www. livingreviews . org//rr-2001-2 


\subsubsection{SZ effect}

The Sunyaev-Zel'dovich (SZ) effect is the change in energy that CMB photons undergo when they scatter in hot gas typically found in cores of galaxy clusters. There are two main effects: thermal and kinetic. Thermal SZ is the dominant mechanism which arises from thermal motion of gas in the temperature range $10^{7}-10^{8} \mathrm{~K}$, and is described by the Compton $y$ parameter

$$
y=\sigma_{\mathrm{T}} \int \frac{n_{\mathrm{e}} k_{\mathrm{B}} T_{\mathrm{e}}}{m_{\mathrm{e}} c^{2}} d l,
$$

where $\sigma_{\mathrm{T}}=6.65 \times 10^{-25} \mathrm{~cm}^{2}$ is the Thomson cross-section, $m_{\mathrm{e}}, n_{\mathrm{e}}$ and $T_{\mathrm{e}}$ are the electron rest mass, density and temperature, $c$ is the speed of light, $k_{\mathrm{B}}$ is Boltzmann's constant, and the integration is performed over the photon path. Photon temperature anisotropies are related to the $y$ parameter by $\Delta T / T \approx-2 y$ in the Rayleigh-Jeans limit. The kinetic SZ effect is a less influential Doppler shift resulting from the bulk motion of ionized gas relative to the rest frame of the CMB.

Springel et al. [150] used a Tree/SPH code to study the SZ effects in a CDM cosmology with a cosmological constant. They find a mean amplitude for thermal SZ $\left(y=3.8 \times 10^{-6}\right)$ just below current observed upper limits, and a kinetic SZ about 30 times smaller in power. Da Silva et al. [66] compared thermal SZ maps in three different cosmologies (low density $+\Lambda$, critical density, and low density open model). Their results are also below current limits: $y \approx 4 \times 10^{-6}$ for low density models with contributions from over a broad redshift range $z \leq 5$, and $y \approx 1 \times 10^{-6}$ for the critical density model with contributions mostly from $z<1$. However, further simulations are needed to explore the dependence of the SZ effect on microphysics, i.e., cooling, star formation, supernovae feedback.

\subsection{Cosmological sheets}

Cosmological sheets, or pancakes, form as overdense regions collapse preferentially along one axis. Originally studied by Zel'dovich [165] in the context of neutrino-dominated cosmologies, sheets are ubiquitous features in nonlinear structure formation simulations of CDM-like models with baryonic fluid, and manifest on a spectrum of length scales and formation epochs. Gas collapses gravitationally into flattened sheet structures, forming two plane parallel shock fronts that propagate in opposite directions, heating the infalling gas. The heated gas between the shocks then cools radiatively and condenses into galactic structures. Sheets are characterized by essentially five distinct components: the preshock inflow, the postshock heated gas, the strongly cooling/recombination front separating the hot gas from the cold, the cooled postshocked gas, and the unshocked adiabatically compressed gas at the center. Several numerical calculations [47, 145, 22] have been performed of these systems which include baryonic fluid with hydrodynamical shock heating, ionization, recombination, dark matter, thermal conductivity, and radiative cooling (Compton, bremsstrahlung, and atomic line cooling), in both one and two spatial dimensions to assert the significance of each physical process and to compute the fragmentation scale. See also [16] where fully general relativistic numerical calculations of cosmological sheets are presented in plane symmetry, including relativistic hydrodynamical shock heating and consistent coupling to spacetime curvature.

In addition, it is well known that gas which cools to $1 \mathrm{eV}$ through hydrogen line cooling will likely cool faster than it can recombine. This nonequilibrium cooling increases the number of electrons and ions (compared to the equilibrium case) which, in turn, increases the concentrations of $\mathrm{H}^{-}$and $\mathrm{H}_{2}^{+}$, the intermediaries that produce hydrogen molecules $\mathrm{H}_{2}$. If large concentrations of molecules form, excitations of the vibrational/rotational modes of the molecules can efficiently cool the gas to well below $1 \mathrm{eV}$, the minimum temperature expected from atomic hydrogen line cooling. Because the gas cools isobarically, the reduction in temperature results in an even greater reduction in the Jeans mass, and the bound objects which form from the fragmentation of $\mathrm{H}_{2}$ 
cooled cosmological sheets may be associated with massive stars or star clusters. Anninos and Norman [18] have carried out 1D and 2D high resolution numerical calculations to investigate the role of hydrogen molecules in the cooling instability and fragmentation of cosmological sheets, considering the collapse of perturbation wavelengths from $1 \mathrm{Mpc}$ to $10 \mathrm{Mpc}$. They find that for the more energetic (long wavelength) cases, the mass fraction of hydrogen molecules reaches $n_{\mathrm{H} 2} / n_{\mathrm{H}} \sim 3 \times 10^{-3}$, which cools the gas to $4 \times 10^{-3} \mathrm{eV}$ and results in a fragmentation scale of $9 \times 10^{3} M_{\odot}$. This represents reductions of 50 and $10^{3}$ in temperature and Jeans mass respectively when compared, as in Figure 12, to the equivalent case in which hydrogen molecules were neglected.

However, the above calculations neglected important interactions arising from self-consistent treatments of radiation fields with ionizing and photo-dissociating photons and self-shielding effects. Susa and Umemura [153] studied the thermal history and hydrodynamical collapse of pancakes in a UV background radiation field. They solve the radiative transfer of photons together with the hydrodynamics and chemistry of atomic and molecular hydrogen species. Although their simulations were restricted to one-dimensional plane parallel symmetry, they suggest a classification scheme distinguishing different dynamical behavior and galaxy formation scenarios based on the UV background radiation level and a critical mass corresponding to $1-2 \sigma$ density fluctuations in a standard CDM cosmology. These level parameters distinguish galaxy formation scenarios as they determine the local thermodynamics, the rate of $\mathrm{H}_{2}$ line emissions and cooling, the amount of starburst activity, and the rate and mechanism of cloud collapse.

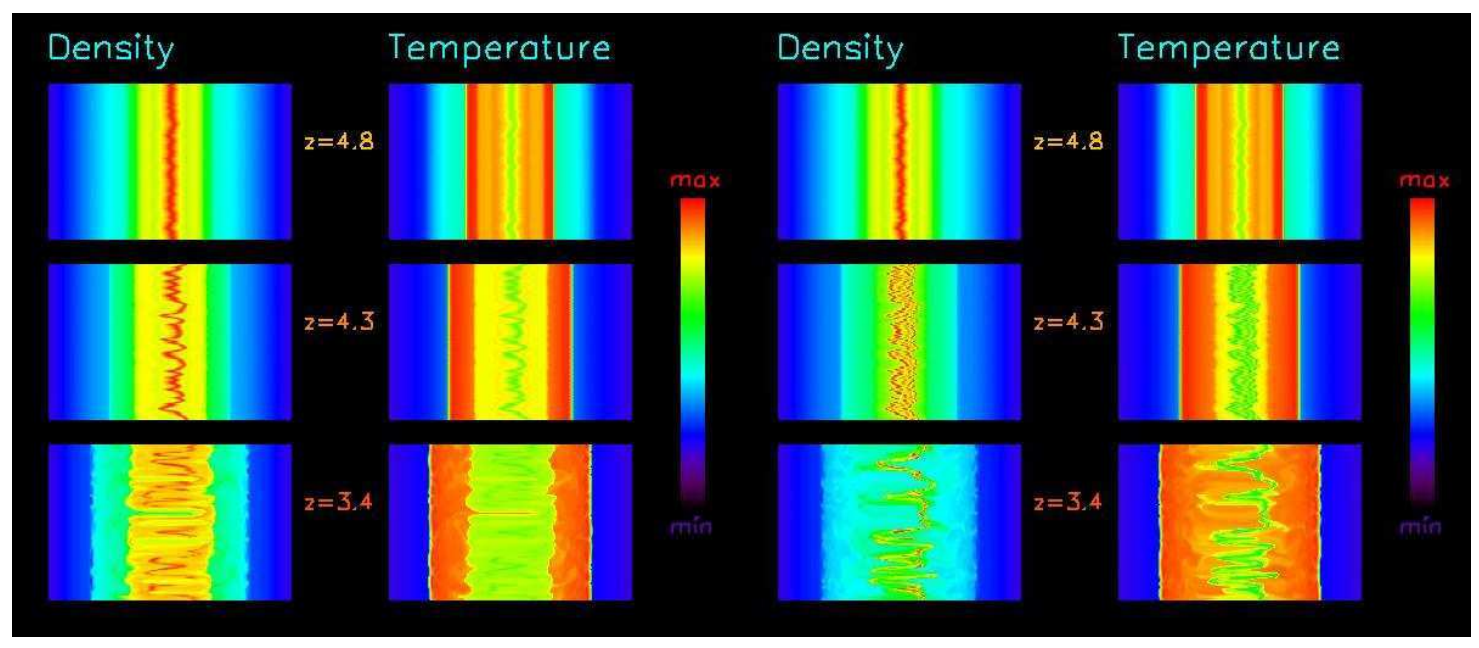

Figure 12: Two different model simulations of cosmological sheets are presented: a six species model including only atomic line cooling (left), and a nine species model including also hydrogen molecules (right). The evolution sequences in the images show the baryonic overdensity and gas temperature at three redshifts following the initial collapse at $z=5$. In each figure, the vertical axis is $32 \mathrm{kpc}$ long (parallel to the plane of collapse) and the horizontal axis extends to $4 \mathrm{Mpc}$ on a logarithmic scale to emphasize the central structures. Differences in the two cases are observed in the cold pancake layer and the cooling flows between the shock front and the cold central layer. When the central layer fragments, the thickness of the cold gas layer in the six (nine) species case grows to 3 (0.3) kpc and the surface density evolves with a dominant transverse mode corresponding to a scale of approximately 8 (1) kpc. Assuming a symmetric distribution of matter along the second transverse direction, the fragment masses are approximately $10^{7}\left(10^{5}\right)$ solar masses.

Living Reviews in Relativity

http://www. livingreviews. org/lrr-2001-2 


\section{Summary}

This review is intended to provide a flavor of the variety of numerical cosmological calculations performed of different events occurring throughout the history of our Universe. The topics discussed range from the strong field dynamical behavior of spacetime geometry at early times near the Big Bang singularity and the epoch of inflation, to the late time evolution of large scale matter fluctuations and the formation of clusters of galaxies. For the most part, the nature of the calculations dealing with the early or late Universe can be distinguished by their basic motivations. For example, calculations of early Universe phenomena are designed to explore alternative cosmological models or topologies, and in some cases, different theories of gravity. They also tend to study the nature of topological singularities, geometric effects, and the problem of initial conditions or the origin of matter distributions. Calculations of the late Universe are generally focused to establish bounds on cosmological parameters in the context of the standard model, to resolve the correct structure formation scenario, to model the complex multi-physics interactions operating at vastly different scales, and to systematically compare invariant measures against observed data for both model validation and interpreting observations.

Although a complete, self-consistent, and accurate description of our Universe is impractical considering the complex multi-scale and multi-physics requirements, a number of enlightening results have been demonstrated through computations. For example, both monotonic AVTD and chaotic oscillatory BLK behavior have been found in the asymptotic approach to the initial singularity in a number of inhomogeneous cosmological models, though some issues remain concerning the generic nature of the singularity, including the effect of nonlinear mode coupling of spatial gradients to the oscillatory history, and the behavior in non-vacuum spacetimes with arbitrary global topology. Numerical calculations suggest that scalar fields play an important complicated role in the nonlinear or chaotic evolution of cosmological models with consequences for the triggering (or not) of inflation and the subsequent dynamics of structure formation. It is possible, for example, that these fields can influence the details of inflation and have observable ramifications as fractal patterns in the density spectrum, gravitational waves, galaxy distribution, and cosmic microwave background anisotropies. All these effects require further studies. Numerical simulations have also been used to place limits on curvature anisotropies and cosmological parameters at early times by considering primordial nucleosynthesis reactions in anisotropic and inhomogeneous cosmologies.

Finally, the large collection of calculations performed of the post-recombination epoch related to large scale structure formation (for example, cosmic microwave background, gravitational lensing, Ly $\alpha$ absorption, and galaxy cluster simulations) have placed strong constraints on the standard model parameters and structure formation scenarios when compared to observations. Considering the range of models consistent with inflation, the preponderance of observational, theoretical and computational data suggest a best fit model of the late structure-forming Universe that is spatially flat with a cosmological constant and a small tilt in the power spectrum. These best fit model parameters, and in particular the introduction of a cosmological constant, are generally consistent with recent evidence of dark energy from supernovae and high precision CMBR observations.

Obviously many fundamental issues remain unresolved, including even the overall shape or topology of the cosmological model which best describes our Universe throughout its entire history. However, the field of numerical cosmology has matured in the development of computational techniques, the modeling of microphysics, and in taking advantage of current trends in computing technologies, to the point that it is now possible to perform high resolution multiphysics simulations and carry out reliable comparisons of numerical models with observational data. 


\section{Appendix: Basic Equations and Numerical Methods}

Some basic equations relevant for fully relativistic as well as perturbative cosmological calculations are summarized in this section, including the complete Einstein equations, choices of kinematical conditions, initial data constraints, stress-energy-momentum tensors, dynamical equations for various matter sources, and the Newtonian counterparts on background isotropic models. References to numerical methods are also provided.

\subsection{The Einstein equations}

\subsubsection{ADM formalism}

There are many ways to write the Einstein equations. The most common is the ADM or $3+1$ form [23] which decomposes spacetime into layers of three-dimensional space-like hypersurfaces, threaded by a time-like normal congruence $n^{\mu}=\left(1,-\beta^{i}\right) / \alpha$, where we use Greek (Latin) indices to specify spacetime (spatial) quantities. The general spacetime metric is written as

$$
d s^{2}=\left(-\alpha^{2}+\beta_{i} \beta^{i}\right) d t^{2}+2 \beta_{i} d x^{i} d t+\gamma_{i j} d x^{i} d x^{j},
$$

where $\alpha$ and $\beta^{i}$ are the lapse function and shift vector respectively, and $\gamma_{i j}$ is the spatial 3-metric. The lapse defines the proper time between consecutive layers of spatial hypersurfaces, the shift propagates the coordinate system from 3-surface to 3-surface, and the induced 3-metric is related to the 4-metric via $\gamma_{\mu \nu}=g_{\mu \nu}+n_{\mu} n_{\nu}$. The Einstein equations are written as four constraint equations,

$$
\begin{aligned}
{ }^{(3)} R+K^{2}-K_{i j} K^{i j} & =16 \pi G \rho_{\mathrm{H}}, \\
\nabla_{i}\left(K^{i j}-\gamma^{i j} K\right) & =8 \pi G s^{j},
\end{aligned}
$$

twelve evolution equations,

$$
\begin{aligned}
\partial_{t} \gamma_{i j} & =\mathcal{L}_{\beta} \gamma_{i j}-2 \alpha K_{i j}, \\
\partial_{t} K_{i j} & =\mathcal{L}_{\beta} K_{i j}-\nabla_{i} \nabla_{j} \alpha+\alpha\left[{ }^{(3)} R_{i j}-2 K_{i k} K_{j}^{k}+K K_{i j}-8 \pi G\left(s_{i j}-\frac{1}{2} s \gamma_{i j}+\frac{1}{2} \rho_{\mathrm{H}} \gamma_{i j}\right)\right],
\end{aligned}
$$

and four kinematical or coordinate conditions for the lapse function and shift vector that can be specified arbitrarily (see Section 6.1.2). Here $K_{i j}$ is the extrinsic curvature describing how the 3 -metric evolves along a time-like normal vector. It is formally defined as the Lie derivative $\left(\mathcal{L}_{n}\right)$ of the spatial metric along the vector $n^{\mu}, K_{i j} \equiv-\mathcal{L}_{n} \gamma_{i j} / 2$. Also,

$$
\begin{aligned}
\mathcal{L}_{\beta} \gamma_{i j} & =\nabla_{i} \beta_{j}+\nabla_{j} \beta_{i}, \\
\mathcal{L}_{\beta} K_{i j} & =\beta^{k} \nabla_{k} K_{i j}+K_{i k} \nabla_{j} \beta^{k}+K_{k j} \nabla_{i} \beta^{k},
\end{aligned}
$$

where $\nabla_{i}$ is the spatial covariant derivative with respect to $\gamma_{i j},{ }^{(3)} R_{i j}$ is the spatial Ricci tensor, $K$ is the trace of the extrinsic curvature $K_{i j}$, and $G$ is the gravitational constant. The matter source terms $\rho_{\mathrm{H}}, s^{j}, s_{i j}$ and $s=s_{i}^{i}$ as seen by the observers at rest in the time slices are obtained from the appropriate projections

$$
\begin{aligned}
\rho_{\mathrm{H}} & =n^{\mu} n^{\nu} T_{\mu \nu}, \\
s_{i} & =-\gamma_{i}^{\mu} n^{\nu} T_{\mu \nu}, \\
s_{i j} & =\gamma_{i}^{\mu} \gamma_{j}^{\nu} T_{\mu \nu}
\end{aligned}
$$

for the energy density, momentum density and spatial stresses, respectively. Here $c=1$, and Greek (Latin) indices refer to 4(3)-dimensional quantities.

Living Reviews in Relativity

http://www. livingreviews.org/lrr-2001-2 
It is worth noting that several alternative formulations of Einstein's equations have been suggested, including hyperbolic systems [136] which have nice mathematical properties, and conformal traceless systems [147, 31] which make use of a conformal decomposition of the 3 -metric and tracefree part of the extrinsic curvature $A_{i j}=K_{i j}-\gamma_{i j} K / 3$. Introducing $\tilde{\gamma}_{i j}=e^{-4 \psi} \gamma_{i j}$ with $e^{4 \psi}=\gamma^{1 / 3}$ so that the determinant of $\tilde{\gamma}_{i j}$ is unity, and $\tilde{A}_{i j}=e^{-4 \psi} A_{i j}$, evolution equations can be written in the conformal traceless system for $\psi, \tilde{\gamma}_{i j}, K, \tilde{A}_{i j}$ and the conformal connection functions, though not all of these variables are independent. However, it is not yet entirely clear which of these methods is best suited for generic problems. For example, hyperbolic forms are easier to characterize mathematically than ADM and may potentially be more stable, but can suffer from greater inaccuracies by introducing additional equations or higher order derivatives. Conformal treatments are considered to be generally more stable [31], but can be less accurate than traditional ADM for short term evolutions [6].

Many numerical methods have been used to solve the Einstein equations, including variants of the leapfrog scheme, the method of McCormack, the two-step Lax-Wendroff method, and the iterative Crank-Nicholson scheme, among others. For a discussion and comparison of the different methods, the reader is referred to [43], where a systematic study was carried out on spherically symmetric black hole spacetimes using traditional ADM, and to [31, 6, 13] (and references therein) which discuss the stability and accuracy of hyperbolic and conformal treatments.

\subsubsection{Kinematic conditions}

For cosmological simulations, one typically takes the shift vector to be zero, hence $\mathcal{L}_{\beta} \gamma_{i j}=\mathcal{L}_{\beta} K_{i j}=$ 0 . However, the shift can be used advantageously in deriving conditions to maintain the 3 -metric in a particular form, and to simplify the resulting differential equations [59,60]. See also [146] describing an approximate minimum distortion gauge condition used to help stabilize simulations of general relativistic binary clusters and neutron stars.

Several options can be implemented for the lapse function, including geodesic $(\alpha=1)$, algebraic, and mean curvature slicing. The algebraic condition takes the form

$$
\alpha=F_{1}\left(x^{\mu}\right) F_{2}(\gamma),
$$

where $F_{1}\left(x^{\mu}\right)$ is an arbitrary function of coordinates $x^{\mu}$, and $F_{2}(\gamma)$ is a dynamic function of the determinant of the 3-metric. This choice is computationally cheap, simple to implement, and certain choices of $F_{2}$ (i.e., $1+\ln \gamma$ ) can mimic maximal slicing in its singularity avoidance properties [8]. On the other hand, numerical solutions derived from harmonically-sliced foliations can exhibit pathological gauge behavior in the form of coordinate "shocks" or singularities which will affect the accuracy, convergence and stability of solutions $[5,86]$. Also, evolutions in which the lapse function is fixed by some analytically prescribed method (either geodesic or near-geodesic) can be unstable, especially for sub-horizon scale perturbations [7].

The mean curvature slicing equation is derived by taking the trace of the extrinsic curvature evolution equation (11),

$$
\nabla^{i} \nabla_{i} \alpha=\alpha\left[K_{i j} K^{i j}+4 \pi G\left(\rho_{\mathrm{H}}+s\right)\right]+\beta^{i} \nabla_{i} K-\partial_{t} K,
$$

and assuming $K=K(t)$, which can either be specified arbitrarily or determined by imposing a boundary condition on the lapse function after solving Equation (17) for the quantity $\alpha / \partial_{t} K[60]$. It is also useful to consider replacing $\partial_{t} K$ in Equation (17) with an exponentially driven form as suggested by Eppley [71], to reduce gauge drifting and numerical errors in maximal [25] and mean curvature [7] sliced spacetimes. The mean curvature slicing condition is the most natural one for cosmology as it foliates homogeneous cosmological spacetimes with surfaces of homogeneity. Also, since $K$ represents the convergence of coordinate curves from one slice to the next and if it is 
constant, then localized caustics (crossing of coordinate curves) and true curvature singularities can be avoided. Whether general inhomogeneous spacetimes can be foliated with constant mean curvature surfaces remains unknown. However, for Gowdy spacetimes with two Killing fields and topology $T^{3} \times R$, Isenberg and Moncrief [97] proved that such foliations do exist and cover the entire spacetime.

\subsubsection{Symplectic formalism}

A different approach to conventional (i.e., $3+1 \mathrm{ADM}$ ) techniques in numerical cosmology has been developed by Berger and Moncrief [40]. For example, they consider Gowdy cosmologies on $T^{3} \times R$ with the metric

$$
d s^{2}=e^{-\lambda / 2} e^{\tau / 2}\left(-e^{-2 \tau} d \tau^{2}+d \theta^{2}\right)+e^{-\tau}\left[e^{P} d \sigma^{2}+2 e^{P} Q d \sigma d \delta+\left(e^{P} Q^{2}+e^{-P}\right) d \delta^{2}\right],
$$

where $\lambda, P$ and $Q$ are functions of $\theta$ and $\tau$, and the coordinates are bounded by $0 \leq(\theta, \sigma, \delta) \leq 2 \pi$. The singularity corresponds to the limit $\tau \rightarrow \infty$. For small amplitudes, $P$ and $Q$ may be identified with + and $\times$ polarized gravitational wave components and $\lambda$ with the background cosmology through which they propagate. An advantage of this formalism is that the initial value problem becomes trivial since $P, Q$ and their first derivatives may be specified arbitrarily (although it is not quite so trivial in more general spacetimes).

Although the resulting Einstein equations can be solved in the usual spacetime discretization fashion, an interesting alternative method of solution is the symplectic operator splitting formulation $[40,121]$ founded on recognizing that the second order equations can be obtained from the variation of a Hamiltonian decomposed into kinetic and potential subhamiltonians,

$$
H=H_{1}+H_{2}=\frac{1}{2} \oint_{0}^{2 \pi} d \theta\left(\pi_{P}^{2}+e^{-2 P} \pi_{Q}^{2}\right)+\frac{1}{2} \oint_{0}^{2 \pi} d \theta e^{-2 \tau}\left(P_{, \theta}^{2}+e^{2 P} Q_{, \theta}^{2}\right) .
$$

The symplectic method approximates the evolution operator by

$$
e^{H \Delta \tau}=e^{H_{2} \Delta \tau / 2} e^{H_{1} \Delta \tau} e^{H_{2} \Delta \tau / 2}+\mathcal{O}(\Delta \tau)^{3},
$$

although higher order representations are possible. If the two Hamiltonian components $H_{1}$ and $\mathrm{H}_{2}$ are each integrable, their solutions can be substituted directly into the numerical evolution to provide potentially more accurate solutions with fewer time steps [36]. This approach is well-suited for studies of singularities if the asymptotic behavior is determined primarily by the kinetic subhamiltonian, a behavior referred to as Asymptotically Velocity Term Dominated (see Section 3.1.2 and [37]).

Symplectic integration methods are applicable to other spacetimes. For example, Berger et al. [39] developed a variation of this approach to explicitly take advantage of exact solutions for scattering between Kasner epochs in Mixmaster models. Their algorithm evolves Mixmaster spacetimes more accurately with larger time steps than previous methods.

\subsubsection{Regge calculus model}

A unique approach to numerical cosmology (and numerical relativity in general) is the method of Regge Calculus in which spacetime is represented as a complex of 4-dimensional, geometrically flat simplices. The principles of Einstein's theory are applied directly to the simplicial geometry to form the curvature, action, and field equations, in contrast to the finite difference approach where the continuum field equations are differenced on a discrete mesh.

A 3-dimensional code implementing Regge Calculus techniques was developed recently by Gentle and Miller [81] and applied to the Kasner cosmological model. They also describe a procedure

Living Reviews in Relativity

http://www. livingreviews.org/lrr-2001-2 
to solve the constraint equations for time asymmetric initial data on two spacelike hypersurfaces constructed from tetrahedra, since full 4-dimensional regions or lattices are used. The new method is analogous to York's procedure (see [163] and Section 6.3) where the conformal metric, trace of the extrinsic curvature, and momentum variables are all freely specifiable. These early results are promising in that they have reproduced the continuum Kasner solution, achieved second order convergence, and sustained numerical stability. Also, Barnett et al. [29] discuss an implicit evolution scheme that allows local (vertex) calculations for efficient parallelism. However, the Regge Calculus approach remains to be developed and applied to more general spacetimes with complex topologies, extended degrees of freedom, and general source terms.

\subsection{Sources of matter}

\subsubsection{Cosmological constant}

A cosmological constant is implemented in the $3+1$ framework simply by introducing the quantity $-\Lambda /(8 \pi G)$ as an effective isotropic pressure in the stress-energy tensor

$$
T_{\mu \nu}=-\frac{\Lambda}{8 \pi G} g_{\mu \nu} .
$$

The matter source terms can then be written as

$$
\begin{aligned}
\rho_{\mathrm{H}} & =\frac{\Lambda}{8 \pi G}, \\
s_{i j} & =-\frac{\Lambda}{8 \pi G} \gamma_{i j},
\end{aligned}
$$

with $s^{i}=0$.

\subsubsection{Scalar field}

The dynamics of scalar fields is governed by the Lagrangian density

$$
\mathcal{L}=-\frac{1}{2} \sqrt{-g}\left[g^{\mu \nu} \nabla_{\mu} \phi \nabla_{\nu} \phi+\xi R f(\phi)+2 V(\phi)\right],
$$

where $R$ is the scalar Riemann curvature, $V(\phi)$ is the interaction potential, $f(\phi)$ is typically assumed to be $f(\phi)=\phi^{2}$, and $\xi$ is the field-curvature coupling constant ( $\xi=0$ for minimally coupled fields and $\xi=1 / 6$ for conformally coupled fields). Varying the action yields the KleinGordon equation

$$
g^{\mu \nu} \nabla_{\mu} \nabla_{\nu} \phi-\xi R \phi-\partial_{\phi} V(\phi)=0,
$$

for the scalar field and

$$
\begin{aligned}
T_{\mu \nu}= & (1-2 \xi) \nabla_{\mu} \phi \nabla_{\nu} \phi+\left(2 \xi-\frac{1}{2}\right) g_{\mu \nu} g^{\sigma \lambda} \nabla_{\sigma} \phi \nabla_{\lambda} \phi \\
& -2 \xi \phi \nabla_{\mu} \nabla_{\nu} \phi+2 \xi \phi g_{\mu \nu} g^{\sigma \lambda} \nabla_{\sigma} \nabla_{\lambda} \phi+\xi G_{\mu \nu} \phi^{2}-g_{\mu \nu} V(\phi),
\end{aligned}
$$

for the stress-energy tensor, where $G_{\mu \nu}=R_{\mu \nu}-g_{\mu \nu} R / 2$.

For a massive, minimally coupled scalar field [46],

$$
T_{\mu \nu}=\partial_{\mu} \phi \partial_{\nu} \phi-\frac{1}{2} g_{\mu \nu} g^{\rho \sigma} \partial_{\rho} \phi \partial_{\sigma} \phi-g_{\mu \nu} V(\phi),
$$


and

$$
\begin{aligned}
\rho_{\mathrm{H}} & =\frac{1}{2} \gamma^{i j} \partial_{i} \phi \partial_{j} \phi+\frac{1}{2} \eta^{2}+V(\phi), \\
s_{i} & =-\eta \partial_{i} \phi \\
s_{i j} & =\gamma_{i j}\left(-\frac{1}{2} \gamma^{k l} \partial_{k} \phi \partial_{l} \phi+\frac{1}{2} \eta^{2}-V(\phi)\right)+\partial_{i} \phi \partial_{j} \phi,
\end{aligned}
$$

where

$$
\eta=n^{\mu} \partial_{\mu} \phi=\frac{1}{\alpha}\left(\partial_{t}-\beta^{k} \partial_{k}\right) \phi
$$

$n^{\mu}=\left(1,-\beta^{i}\right) / \alpha$, and $V(\phi)$ is a general potential which, for example, can be set to $V=\lambda \phi^{4}$ in the chaotic inflation model. The covariant form of the scalar field equation (25) can be expanded as in [107] to yield

$$
\frac{1}{\alpha}\left(\partial_{t}-\beta^{k} \partial_{k}\right) \eta=\frac{1}{\sqrt{\gamma}} \partial_{i}\left(\sqrt{\gamma} \gamma^{i j} \partial_{j} \phi\right)+\frac{1}{\alpha} \gamma^{i j} \partial_{i} \alpha \partial_{j} \phi+K \eta-\partial_{\phi} V(\phi),
$$

which, when coupled to Equation (31), determines the evolution of the scalar field.

\subsubsection{Collisionless dust}

The stress-energy tensor for a fluid composed of collisionless particles (or dark matter) can be written simply as the sum of the stress-energy tensors for each particle [161],

$$
T_{\mu \nu}=\sum m n u_{\mu} u_{\nu}
$$

where $m$ is the rest mass of the particles, $n$ is the number density in the comoving frame, and $u^{\mu}$ is the 4 -velocity of each particle. The matter source terms are

$$
\begin{aligned}
\rho_{\mathrm{H}} & =\sum m n\left(\alpha u^{0}\right)^{2}, \\
s_{i} & =\sum m n u_{i}\left(\alpha u^{0}\right), \\
s_{i j} & =\sum m n u_{i} u_{j} .
\end{aligned}
$$

There are two conservation laws: the conservation of particles $\nabla_{\mu}\left(n u^{\mu}\right)=0$, and the conservation of energy-momentum $\nabla_{\mu} T^{\mu \nu}=0$, where $\nabla_{\mu}$ is the covariant derivative in the full 4-dimensional spacetime. Together these conservation laws lead to $u^{\mu} \nabla_{\mu} u^{\nu}=0$, the geodesic equations of motion for the particles, which can be written out more explicitly in the computationally convenient form

$$
\begin{aligned}
\frac{d x^{i}}{d t} & =\frac{g^{i \alpha} u_{\alpha}}{u^{0}} \\
\frac{d u_{i}}{d t} & =-\frac{u_{\alpha} u_{\beta} \partial_{i} g^{\alpha \beta}}{2 u^{0}},
\end{aligned}
$$

where $x^{i}$ is the coordinate position of each particle, $u^{0}$ is determined by the normalization $u^{\mu} u_{\mu}=$ $-1$

$$
\frac{d}{d t} \equiv v^{\mu} \partial_{\mu}=\partial_{t}+v^{i} \partial_{i}
$$

is the Lagrangian derivative, and $v^{\mu}=u^{\mu} / u^{0}$ is the "transport" velocity of the particles as measured by observers at rest with respect to the coordinate grid.

Living Reviews in Relativity

http: //www. livingreviews.org/Irr-2001-2 


\subsubsection{Ideal gas}

The stress-energy tensor for a perfect fluid is

$$
T_{\mu \nu}=\rho h u_{\mu} u_{\nu}+P g_{\mu \nu},
$$

where $g_{\mu \nu}$ is the 4-metric, $h=1+\epsilon+P / \rho$ is the relativistic enthalpy, and $\epsilon, P, \rho$ and $u_{\mu}$ are the specific internal energy (per unit mass), pressure, rest mass density and four-velocity of the fluid. Defining $V^{i}=u^{i} / u^{0}$ and

$$
u=-n_{\mu} u^{\mu}=\alpha u^{0}=\left(1+u^{i} u_{i}\right)^{1 / 2}=\left(1-\frac{V_{i} V^{i}}{\alpha^{2}}\right)^{-1 / 2},
$$

as the generalization of the special relativistic boost factor, the matter source terms become

$$
\begin{aligned}
\rho_{\mathrm{H}} & =\rho h u^{2}-P, \\
s_{i} & =\rho h u u_{i}, \\
s_{i j} & =P \gamma_{i j}+\rho h u_{i} u_{j} .
\end{aligned}
$$

The hydrodynamics equations are derived from the normalization of the 4 -velocity, $u^{\mu} u_{\mu}=-1$, the conservation of baryon number, $\nabla_{\mu}\left(\rho u^{\mu}\right)=0$, and the conservation of energy-momentum, $\nabla_{\mu} T^{\mu \nu}=0$. The resulting equations can be written in flux conservative form as [162]

$$
\begin{array}{r}
\frac{\partial D}{\partial t}+\frac{\partial\left(D V^{i}\right)}{\partial x^{i}}=0, \\
\frac{\partial E}{\partial t}+\frac{\partial\left(E V^{i}\right)}{\partial x^{i}}+P \frac{\partial W}{\partial t}+P \frac{\partial\left(W V^{i}\right)}{\partial x^{i}}=0, \\
\frac{\partial S_{i}}{\partial t}+\frac{\partial\left(S_{i} V^{j}\right)}{\partial x^{j}}-\frac{S^{\mu} S^{\nu}}{2 S^{0}} \frac{\partial g_{\mu \nu}}{\partial x^{i}}+\sqrt{-g} \frac{\partial P}{\partial x^{i}}=0,
\end{array}
$$

where $W=\sqrt{-g} u^{0}, D=W \rho, E=W \rho \epsilon, S_{i}=W \rho h u_{i}$, and $g$ is the determinant of the 4-metric satisfying $\sqrt{-g}=\alpha \sqrt{\gamma}$. A prescription for specifying an equation of state (e.g., $P=(\Gamma-1) E / W=$ $(\Gamma-1) \rho \epsilon$ for an ideal gas) completes the above equations.

When solving Equations $(45,46,47)$, an artificial viscosity method is needed to handle the formation and propagation of shock fronts [162, 85, 84]. These methods are computationally cheap, easy to implement, and easily adaptable to multi-physics applications. However, it has been demonstrated that problems involving very high Lorentz factors are somewhat sensitive to different implementations of the viscosity terms, and can result in substantial numerical errors if solved using time explicit methods [126].

On the other hand, a number of different formulations [75] of these equations have been developed to take advantage of the hyperbolic and conservative nature of the equations in using high resolution and non-oscillatory shock capturing schemes (although strict conservation is only possible in flat spacetimes - curved spacetimes exhibit source terms due to geometry). These techniques potentially provide more accurate and stable treatments in highly relativistic regimes. A particular formulation used together with high resolution Godunov techniques and approximate Riemann solvers is the following $[139,26]$ :

$$
\frac{\partial \sqrt{\gamma} U(\vec{w})}{\partial t}+\frac{\partial \sqrt{-g} F^{i}(\vec{w})}{\partial x^{i}}=\sqrt{-g} S(\vec{w}),
$$

where

$$
\begin{aligned}
S(\vec{w}) & =\left[0, T^{\mu \nu}\left(\frac{\partial g_{\nu j}}{\partial x^{\mu}}-\Gamma_{\nu \mu}^{\delta} g_{\delta j}\right), \alpha\left(T^{\mu 0} \frac{\partial \ln \alpha}{\partial x^{\mu}}-T^{\mu \nu} \Gamma_{\nu \mu}^{0}\right)\right], \\
F^{i}(\vec{w}) & =\left[D\left(v^{i}-\frac{\beta^{i}}{\alpha}\right), S_{j}\left(v^{i}-\frac{\beta^{i}}{\alpha}\right)+P \delta_{j}^{i},(E-D)\left(v^{i}-\frac{\beta^{i}}{\alpha}\right)+P v^{i}\right],
\end{aligned}
$$


and $\vec{w}=\left(\rho, v_{i}, \epsilon\right), U(\vec{w})=\left(D, S_{i}, E-D\right), E=\rho h \tilde{W}^{2}-P, S_{j}=\rho h \tilde{W}^{2} v_{j}, D=\rho \tilde{W}, v^{i}=\gamma^{i j} v_{j}=$ $u^{i} /\left(\alpha u^{0}\right)+\beta^{i} / \alpha$, and $\tilde{W}=\alpha u^{0}=\left(1-\gamma_{i j} v^{i} v^{j}\right)^{-1 / 2}$.

Although Godunov-type schemes are accepted as more accurate alternatives to AV methods, especially in the limit of high Lorentz factors, they are not immune to problems and should generally be used with caution. They may produce unexpected results in certain cases that can be overcome only with problem-specific fixes or by adding additional dissipation. A few known examples include the admittance of expansion shocks, negative internal energies in kinematically dominated flows, the 'carbuncle' effect in high Mach number bow shocks, kinked Mach stems, and odd/even decoupling in mesh-aligned shocks [135]. Godunov methods, whether they solve the Riemann problem exactly or approximately, are also computationally much more expensive than their simpler AV counterparts, and it is more difficult to incorporate additional physics.

A third class of computational fluid dynamics methods reviewed here is also based on a conservative hyperbolic formulation of the hydrodynamics equations. However, in this case the equations are derived directly from the conservation of stress-energy,

$$
\nabla_{\mu} T^{\mu \nu}=\frac{1}{\sqrt{-g}} \partial_{\mu}\left(\sqrt{-g} T^{\mu \nu}\right)+\Gamma_{\alpha \mu}^{\nu} T^{\mu \alpha}=0,
$$

to give

$$
\begin{aligned}
\frac{\partial D}{\partial t}+\frac{\partial\left(D V^{i}\right)}{\partial x^{i}} & =0, \\
\frac{\partial \mathcal{E}}{\partial t}+\frac{\partial\left(\mathcal{E} V^{i}\right)}{\partial x^{i}}+\frac{\partial\left[\sqrt{-g}\left(g^{0 i}-g^{00} V^{i}\right) P\right]}{\partial x^{i}} & =\Sigma^{0}, \\
\frac{\partial \mathcal{S}^{j}}{\partial t}+\frac{\partial\left(\mathcal{S}^{j} V^{i}\right)}{\partial x^{i}}+\frac{\partial\left[\sqrt{-g}\left(g^{i j}-g^{0 j} V^{i}\right) P\right]}{\partial x^{i}} & =\Sigma^{j},
\end{aligned}
$$

with curvature source terms $\Sigma^{\nu}=-\sqrt{-g} T^{\beta \gamma} \Gamma_{\beta \gamma}^{\nu}$. The variables $D$ and $V^{i}$ are the same as those defined in the internal energy formulation, but now

$$
\begin{aligned}
\mathcal{E} & =W \rho h u^{0}+\sqrt{-g} g^{00} P, \\
\mathcal{S}^{i} & =W \rho h u^{i}+\sqrt{-g} g^{0 i} P
\end{aligned}
$$

are different expressions for energy and momenta. An alternative approach of using high resolution, non-oscillatory, central difference (NOCD) methods [99, 100] has been applied by Anninos and Fragile [12] to solve the relativistic hydrodynamics equations in the above form. These schemes combine the speed, efficiency, and flexibility of AV methods with the advantages of the potentially more accurate conservative formulation approach of Godunov methods, but without the cost and complication of Riemann solvers and flux splitting.

NOCD and artificial viscosity methods have been discussed at length in [12] and compared also with other published Godunov methods on their abilities to model shock tube, wall shock and black hole accretion problems. They find that for shock tube problems at moderate to high boost factors, with velocities up to $V \sim 0.99$, internal energy formulations using artificial viscosity methods compare quite favorably with total energy schemes, including NOCD methods and Godunov methods using either approximate or exact Riemann solvers. However, AV methods can be somewhat sensitive to parameters (e.g., viscosity coefficients, Courant factor, etc.) and generally suspect in wall shock problems at high boost factors $(V>0.95)$. On the other hand, NOCD methods can easily be extended to ultra-relativistic velocities $\left(1-V<10^{-11}\right)$ for the same wall shock tests, and are comparable in accuracy to the more standard but complicated Riemann solver codes. NOCD schemes thus provide a reasonable alternative for relativistic hydrodynamics, though it should be noted that low order versions of these methods can be significantly more diffusive than either the AV or Godunov methods.

Living Reviews in Relativity

http://www. livingreviews . org/lrr-2001-2 


\subsubsection{Imperfect fluid}

The perfect fluid equations discussed in Section 6.2.4 can be generalized to include viscous stress in the stress-energy tensor,

$$
\begin{aligned}
T^{\mu \nu} & =\left[T_{\text {ideal }}^{\mu \nu}\right]+\left[T_{\text {viscous }}^{\mu \nu}\right] \\
& =\left[\rho h u^{\mu} u^{\nu}+P g^{\mu \nu}\right]-\left[2 \eta \sigma^{\mu \nu}+\xi \theta\left(g^{\mu \nu}+u^{\mu} u^{\nu}\right)\right],
\end{aligned}
$$

where $\eta \geq 0$ and $\xi \geq 0$ are the dynamic shear and bulk viscosity coefficients, respectively. Also, $\theta=\nabla_{\mu} u^{\mu}$ is the expansion of fluid world lines, $\sigma^{\mu \nu}$ is the trace-free spatial shear tensor with

$$
\sigma^{\mu \nu}=\frac{1}{2}\left(h^{\lambda \nu} \nabla_{\lambda} u^{\mu}+h^{\lambda \mu} \nabla_{\lambda} u^{\nu}\right)-\frac{1}{3} \theta h^{\mu \nu},
$$

and $h^{\mu \nu}=g^{\mu \nu}+u^{\mu} u^{\nu}$ is the projection tensor.

The corresponding energy and momentum conservation equations for the internal energy formulation of Section 6.2.4 become

$$
\begin{gathered}
\frac{\partial E}{\partial t}+P \frac{\partial W}{\partial t}+\frac{\partial\left(E v^{i}\right)}{\partial x^{i}}+P \frac{\partial\left(W v^{i}\right)}{\partial x^{i}}=\sqrt{-g} u_{\nu} \nabla_{\mu} T_{\text {viscous }}^{\mu \nu} \\
\frac{\partial S_{j}}{\partial t}+\frac{\partial\left(S_{j} v^{i}\right)}{\partial x^{i}}+\sqrt{-g} \frac{\partial P}{\partial x^{j}}-\frac{S^{\mu} S^{\nu}}{2 S^{0}} \frac{\partial g_{\mu \nu}}{\partial x^{j}}=-\sqrt{-g} g_{j \nu} \nabla_{\mu} T_{\text {viscous }}^{\mu \nu} .
\end{gathered}
$$

For the NOCD formulation discussed in Section 6.2.4 it is sufficient to replace the source terms in the energy and momentum equations $(53,53,54)$ by

$$
\Sigma^{\alpha}=-\sqrt{-g} T^{\beta \gamma} \Gamma_{\beta \gamma}^{\alpha}-\frac{\partial}{\partial t}\left(\sqrt{-g} T_{\text {viscous }}^{0 \alpha}\right)-\frac{\partial}{\partial x^{j}}\left(\sqrt{-g} T_{\text {viscous }}^{j \alpha}\right) .
$$

\subsection{Constrained nonlinear initial data}

One cannot take arbitrary data to initiate an evolution of the Einstein equations. The data must satisfy the constraint equations (9) and (10). York [163] developed a procedure to generate proper initial data by introducing conformal transformations of the 3-metric $\gamma_{i j}=\psi^{4} \hat{\gamma}_{i j}$, the trace-free momentum components $A^{i j}=K^{i j}-\gamma^{i j} K / 3=\psi^{-10} \hat{A}^{i j}$, and matter source terms $s^{i}=\psi^{-10} \hat{s}^{i}$ and $\rho_{\mathrm{H}}=\psi^{-n} \hat{\rho}_{\mathrm{H}}$, where $n>5$ for uniqueness of solutions to the elliptic equation (63) below. In this procedure, the conformal (or "hatted") variables are freely specifiable. Further decomposing the free momentum variables into transverse and longitudinal components $\hat{A}^{i j}=\hat{A}_{*}^{i j}+(\hat{l} w)^{i j}$, the Hamiltonian and momentum constraints are written as

$$
\begin{aligned}
\hat{\nabla}_{i} \hat{\nabla}^{i} \psi-\frac{\hat{R}}{8} \psi+\frac{1}{8} \hat{A}_{i j} \hat{A}^{i j} \psi^{-7}-\frac{1}{12} K^{2} \psi^{5}+2 \pi G \hat{\rho} \psi^{5-n} & =0, \\
\left(\hat{\nabla}_{j} \hat{\nabla}^{j} w\right)^{i}+\frac{1}{3} \hat{\nabla}^{i}\left(\hat{\nabla}_{j} w^{j}\right)+\hat{R}_{j}^{i} w^{j}-\frac{2}{3} \psi^{6} \hat{\nabla}^{i} K-8 \pi G \hat{s}^{i} & =0,
\end{aligned}
$$

where the longitudinal part of $\hat{A}^{i j}$ is reconstructed from the solutions by

$$
(\hat{l} w)^{i j}=\hat{\nabla}^{i} w^{j}+\hat{\nabla}^{j} w^{i}-\frac{2}{3} \hat{\gamma}^{i j} \hat{\nabla}_{k} w^{k}
$$

The transverse part of $\hat{A}^{i j}$ is constrained to satisfy $\hat{\nabla}_{j} \hat{A}_{*}^{i j}=\hat{A}_{* j}^{j}=0$.

Equations (63) and (64) form a coupled nonlinear set of elliptic equations which must be solved iteratively, in general. The two equations can, however, be decoupled if a mean curvature slicing $(K=K(t))$ is assumed. Given the free data $K, \hat{\gamma}_{i j}, \hat{s}^{i}$ and $\hat{\rho}$, the constraints are solved for $\hat{A}_{*}^{i j}$, 
$(\hat{l} w)^{i j}$ and $\psi$. The actual metric $\gamma_{i j}$ and curvature $K_{i j}$ are then reconstructed by the corresponding conformal transformations to provide the complete initial data. Anninos [7] describes a procedure using York's formalism to construct parametrized inhomogeneous initial data in freely specifiable background spacetimes with matter sources. The procedure is general enough to allow gravitational wave and Coulomb nonlinearities in the metric, longitudinal momentum fluctuations, isotropic and anisotropic background spacetimes, and can accommodate the conformal-Newtonian gauge to set up gauge invariant cosmological perturbation solutions as free data.

\subsection{Newtonian limit}

The Newtonian limit is defined by spatial scales much smaller than the horizon radius, peculiar velocities small compared to the speed of light, and a gravitational potential that is both much smaller than unity (in geometric units) and slowly varying in time. A comprehensive review of the theory of cosmological perturbations can be found in [124].

\subsubsection{Dark and baryonic matter equations}

The appropriate perturbation equations in this limit are easily derived for a background FLRW expanding model, assuming a metric of the form

$$
d s^{2}=-(1+2 \Phi) d t^{2}+a(t)^{2}(1-2 \Phi) \gamma_{i j} d x^{i} d x^{j},
$$

where

$$
\gamma_{i j}=\delta_{j}^{i}\left(1+\frac{k r^{2}}{4}\right)^{-2}
$$

and $k=-1,0,+1$ for open, flat and closed Universes. Also, $a \equiv 1 /(1+z)$ is the cosmological scale factor, $z$ is the redshift, and $\Phi$ is the comoving inhomogeneous gravitational potential.

The governing equations in the Newtonian limit are the hydrodynamic conservation equations,

$$
\begin{array}{r}
\frac{\partial \tilde{\rho}_{\mathrm{b}}}{\partial t}+\frac{\partial}{\partial x^{i}}\left(\tilde{\rho}_{\mathrm{b}} v_{\mathrm{b}}^{i}\right)+3 \frac{\dot{a}}{a} \tilde{\rho}_{\mathrm{b}}=0, \\
\frac{\partial\left(\tilde{\rho}_{\mathrm{b}} v_{\mathrm{b}}^{j}\right)}{\partial t}+\frac{\partial}{\partial x^{i}}\left(\tilde{\rho}_{\mathrm{b}} v_{\mathrm{b}}^{i} v_{\mathrm{b}}^{j}\right)+5 \frac{\dot{a}}{a} \tilde{\rho}_{\mathrm{b}} v_{\mathrm{b}}^{j}+\frac{1}{a^{2}} \frac{\partial \tilde{p}}{\partial x^{j}}+\frac{\tilde{\rho}_{\mathrm{b}}}{a^{2}} \frac{\partial \tilde{\Phi}}{\partial x^{j}}=0, \\
\frac{\partial \tilde{e}}{\partial t}+\frac{\partial}{\partial x^{i}}\left(\tilde{e} v_{\mathrm{b}}^{i}\right)+\tilde{p} \frac{\partial v_{\mathrm{b}}^{i}}{\partial x^{i}}+3 \frac{\dot{a}}{a}(\tilde{e}+\tilde{p})=\tilde{S}_{\mathrm{cool}},
\end{array}
$$

the geodesic equations for collisionless dust or dark matter (in comoving coordinates),

$$
\begin{aligned}
& \frac{d x_{\mathrm{d}}^{i}}{d t}=v_{\mathrm{d}}^{i}, \\
& \frac{d v_{\mathrm{d}}^{i}}{d t}=-2 \frac{\dot{a}}{a} v_{\mathrm{d}}^{i}-\frac{1}{a^{2}} \frac{\partial \tilde{\Phi}}{\partial x^{i}},
\end{aligned}
$$

Poisson's equation for the gravitational potential,

$$
\nabla^{2} \tilde{\Phi}=4 \pi G a^{2}\left(\tilde{\rho}_{\mathrm{b}}+\tilde{\rho}_{\mathrm{d}}-\tilde{\rho}_{0}\right),
$$

and the Friedman equation for the cosmological scale factor,

$$
\frac{d a}{d t}=H_{0}\left[\Omega_{\mathrm{m}}\left(\frac{1}{a}-1\right)+\Omega_{\Lambda}\left(a^{2}-1\right)+1\right]^{1 / 2} .
$$

Living Reviews in Relativity

http: //www. livingreviews.org/Irr-2001-2 
Here $\tilde{\rho}_{\mathrm{d}}, \tilde{\rho}_{\mathrm{b}}, \tilde{p}$ and $\tilde{e}$ are the dark matter density, baryonic density, pressure and internal energy density in the proper reference frame, $x^{i}$ and $v_{\mathrm{b}}^{i}$ are the baryonic comoving coordinates and peculiar velocities, $x_{\mathrm{d}}^{i}$ and $v_{\mathrm{d}}^{i}$ are the dark matter comoving coordinates and peculiar velocities, $\tilde{\rho}_{0}=3 H_{0}^{2} \Omega_{0} /\left(8 \pi G a^{3}\right)$ is the proper background density of the Universe, $\Omega_{0}$ is the total density parameter, $\Omega_{\mathrm{m}}=\Omega_{\mathrm{b}}+\Omega_{\mathrm{d}}$ is the density parameter including both baryonic and dark matter contributions, $\Omega_{\Lambda}=\Lambda /\left(3 H_{0}^{2}\right)$ is the density parameter attributed to the cosmological constant $\Lambda$, $H_{0}=100 h \mathrm{~km} \mathrm{~s}^{-1} \mathrm{Mpc}^{-1}$ is the present Hubble constant with $0.5<h<1$, and $\tilde{S}_{\text {cool }}$ represents microphysical radiative cooling and heating rates which can include Compton cooling (or heating) due to interactions of free electrons with the CMBR, bremsstrahlung, and atomic and molecular line cooling. Notice that 'tilded' ('non-tilded') variables refer to proper (comoving) reference frame attributes.

An alternative total energy conservative form of the hydrodynamics equations that allows high resolution Godunov-type shock capturing techniques is

$$
\begin{aligned}
\frac{\partial \rho_{\mathrm{b}}}{\partial t}+\frac{1}{a} \frac{\partial}{\partial x^{i}}\left(\rho_{\mathrm{b}} \tilde{v}_{\mathrm{b}}^{i}\right) & =0, \\
\frac{\partial\left(\rho_{\mathrm{b}} \tilde{v}_{\mathrm{b}}^{j}\right)}{\partial t}+\frac{1}{a} \frac{\partial}{\partial x^{i}}\left(\rho_{\mathrm{b}} \tilde{v}_{\mathrm{b}}^{i} \tilde{v}_{\mathrm{b}}^{j}+p \delta_{i j}\right)+\frac{\dot{a}}{a} \rho_{\mathrm{b}} \tilde{v}_{\mathrm{b}}^{j}+\frac{\rho_{\mathrm{b}}}{a} \frac{\partial \tilde{\Phi}}{\partial x^{j}} & =0, \\
\frac{\partial E}{\partial t}+\frac{1}{a} \frac{\partial}{\partial x^{i}}\left(E \tilde{v}_{\mathrm{b}}^{i}+p \tilde{v}_{\mathrm{b}}^{i}\right)+\frac{2 \dot{a}}{a} E+\frac{\rho_{\mathrm{b}} \tilde{v}_{\mathrm{b}}^{i}}{a} \frac{\partial \tilde{\Phi}}{\partial x^{i}} & =S_{\text {cool },}
\end{aligned}
$$

with the corresponding particle and gravity equations

$$
\begin{aligned}
\frac{d x_{\mathrm{d}}^{i}}{d t} & =\frac{\tilde{v}_{\mathrm{d}}^{i}}{a}, \\
\frac{d \tilde{v}_{\mathrm{d}}^{i}}{d t} & =-\frac{\dot{a}}{a} \tilde{v}_{\mathrm{d}}^{i}-\frac{1}{a} \frac{\partial \tilde{\Phi}}{\partial x^{i}}, \\
\nabla^{2} \tilde{\Phi} & =\frac{4 \pi G}{a}\left(\rho_{\mathrm{b}}+\rho_{\mathrm{d}}-\rho_{0}\right),
\end{aligned}
$$

where $\rho_{\mathrm{b}}$ is the comoving density, $\rho_{0}=a^{3} \tilde{\rho}_{0}$, $\tilde{v}_{\mathrm{b}}^{i}$ is the proper frame peculiar velocity, $p$ is the comoving pressure, $E=\rho_{b} \tilde{v}_{b}^{2} / 2+p /(\gamma-1)$ is the total peculiar energy per comoving volume, and $\tilde{\Phi}$ is the gravitational potential.

\subsubsection{Primordial chemistry}

The baryonic equations from the previous section are easily extended to include reactive chemistry of both atomic and molecular species (e.g., $\mathrm{H}, \mathrm{H}^{+}, \mathrm{He}, \mathrm{He}^{+}, \mathrm{He}^{++}, \mathrm{H}^{-}, \mathrm{H}_{2}^{+}, \mathrm{H}_{2}$, and $\mathrm{e}^{-}$) by assuming a common flow field, supplementing the total mass conservation equation (68) with

$$
\frac{\partial \tilde{\rho}_{j}}{\partial t}+\frac{\partial}{\partial x^{i}}\left(\tilde{\rho}_{j} v_{\mathrm{b}}^{i}\right)+3 \frac{\dot{a}}{a} \tilde{\rho}_{j}=\sum_{i} \sum_{l} k_{i l}(T) \tilde{\rho}_{i} \tilde{\rho}_{l}+\sum_{i} I_{i} \tilde{\rho}_{i}
$$

for each of the species, and including the effects of non-equilibrium radiative cooling and consistent coupling to the hydrodynamics equations. The $k_{i l}(T)$ are rate coefficients for the two body reactions and are generally incorporated in numerical codes as tabulated functions of the gas temperature $T$. The $I_{i}$ are integrals evaluating the photoionization and photodissociation of the different species.

A fairly complete chemical network system useful for primordial gas phase compositions, including hydrogen molecules, consists of the following collisional, photoionization, and photodissociation chains 
- Collisional reactions (primordial chain):
(1) $\mathrm{H}+e \rightarrow \mathrm{H}^{+}+2 e$
(2) $\mathrm{H}+e \rightarrow \mathrm{H}^{+}+2 e$
(3) $\mathrm{H}+e \rightarrow \mathrm{H}^{+}+2 e$
(4) $\mathrm{H}+e \rightarrow \mathrm{H}^{+}+2 e$
(5) $\mathrm{He}^{+}+e \rightarrow \mathrm{He}^{++}+2 e$
(6) $\mathrm{He}^{++}+e \rightarrow \mathrm{He}^{+}+\gamma$

- Collisional reactions $\left(\mathrm{H}_{2}\right.$ molecular chain):
(7) $\mathrm{H}+e \rightarrow \mathrm{H}^{-}+\gamma$
(8) $\mathrm{H}+e \rightarrow \mathrm{H}^{-}+\gamma$
(9) $\mathrm{H}+e \rightarrow \mathrm{H}^{-}+\gamma$
(10) $\mathrm{H}+e \rightarrow \mathrm{H}^{-}+\gamma$
(11) $\mathrm{H}+e \rightarrow \mathrm{H}^{-}+\gamma$
(12) $\mathrm{H}+e \rightarrow \mathrm{H}^{-}+\gamma$
(13) $\mathrm{H}+e \rightarrow \mathrm{H}^{-}+\gamma$
(14) $\mathrm{H}+e \rightarrow \mathrm{H}^{-}+\gamma$
(15) $\mathrm{H}+e \rightarrow \mathrm{H}^{-}+\gamma$
(16) $\mathrm{H}+e \rightarrow \mathrm{H}^{-}+\gamma$
(17) $\mathrm{H}+e \rightarrow \mathrm{H}^{-}+\gamma$
(18) $\mathrm{H}_{2}^{+}+\mathrm{H}^{-} \rightarrow \mathrm{H}_{2}+\mathrm{H}$
(19) $\mathrm{H}_{2}^{+}+\mathrm{H}^{-} \rightarrow \mathrm{H}_{2}+\mathrm{H}$

- Photoionization reactions (primordial chain):$$
\mathrm{H}+\gamma \rightarrow \mathrm{H}^{+}+e
$$

$$
\mathrm{He}+\gamma \rightarrow \mathrm{He}^{+}+e
$$$$
\mathrm{He}^{+}+\gamma \rightarrow \mathrm{He}^{++}+e
$$

- Photodissociation/ionization reactions (molecular chain):

$$
\begin{array}{ll}
(23) & \mathrm{H}^{-}+\gamma \rightarrow \mathrm{H}+e \\
(24) & \mathrm{H}^{-}+\gamma \rightarrow \mathrm{H}+e \\
(25) & \mathrm{H}^{-}+\gamma \rightarrow \mathrm{H}+e \\
(26) & \mathrm{H}^{-}+\gamma \rightarrow \mathrm{H}+e \\
(27) & \mathrm{H}_{2}+\gamma \rightarrow 2 \mathrm{H}
\end{array}
$$$$
\text { (26) } \quad \mathrm{H}^{-}+\gamma \rightarrow \mathrm{H}+e
$$

For a comprehensive description of the chemistry and explicit formulas modeling the kinetic and cooling rates relevant for cosmological calculations, the reader is referred to [92, 144, 54, 1, 21]. This reactive network is by no means complete, and in fact, ignores important coolants and contaminants (e.g., HD, LiH, and their intermediary products [151, 78, 48]) expected to form through nonequilibrium reactions at low temperatures and high densities. Although it is certainly possible to include even in three dimensional simulations, the inclusion of more complex reactants demands either more computational resources (to resolve both the chemistry and cooling scales) or an increasing reliance on equilibrium assumptions which can be very inaccurate.

Living Reviews in Relativity

http: //www. livingreviews. org/lrr-2001-2 


\subsubsection{Numerical methods}

Many numerical techniques have been developed to solve the hydrodynamic and collisionless particle equations. For the hydrodynamic equations, the methods range from Lagrangian SPH algorithms with artificial viscosity [72,88], to high resolution shock capturing Eulerian techniques on single static meshes [142, 134], nested grids [19], moving meshes [82], and adaptive mesh refinement [51]. For the dark matter equations, the canonical choices are treecodes [159] or PM and $\mathrm{P}^{3} \mathrm{M}$ methods [90,68], although many variants have been developed to optimize computational performance and accuracy, including adaptive mesh, particle, and smoothing kernel refinement methods $[45,77,130]$. An efficient method for solving non-equilibrium, multi-species chemical reactive flows together with the hydrodynamic equations in a background FLRW model is described in $[1,21]$.

It is beyond the scope of this review to discuss algorithmic details of the different methods and their strengths and weaknesses. Instead, the reader is referred to $[103,77]$ for thorough comparisons of various numerical methods applied to problems of structure formation. Kang et al. [103] compare (by binning data at different resolutions) the statistical performance of five codes (three Eulerian and two SPH) on the problem of an evolving CDM Universe on large scales using the same initial data. The results indicate that global averages of physical attributes converge in rebinned data, but that some uncertainties remain at small levels. Frenk et al. [77] compare twelve Lagrangian and Eulerian hydrodynamics codes to resolve the formation of a single X-ray cluster in a CDM Universe. The study finds generally good agreement for both dynamical and thermodynamical quantities, but also shows significant differences in the X-ray luminosity, a quantity that is especially sensitive to resolution [17].

\subsubsection{Linear initial data}

The standard Zel'dovich solution [165, 68] is commonly used to generate initial conditions satisfying observed or theoretical power spectra of matter density fluctuations. Comoving physical displacements and velocities of the collisionless dark matter particles are set according to the power spectrum realization

$$
\left|\frac{\delta \rho}{\rho}(k)\right|^{2} \propto k^{n} T^{2}(k),
$$

where the complex phases are chosen from a gaussian random field, $T(k)$ is a transfer function [27] appropriate to a particular structure formation scenario (e.g., CDM), and $n=1$ corresponds to the Harrison-Zel'dovich power spectrum. The fluctuations are normalized with top hat smoothing using

$$
\sigma_{8}^{2}=\frac{1}{b^{2}}=\int_{0}^{\infty} 4 \pi k^{2} P(k) W^{2}(k) d k,
$$

where $b$ is the bias factor chosen to match present observations of rms density fluctuations in a spherical window of radius $R_{\mathrm{h}}=8 h^{-1}$ Mpc. Also, $P(k)$ is the Fourier transform of the square of the density fluctuations in Equation (82), and

$$
W(k)=\frac{3}{\left(k R_{\mathrm{h}}\right)^{3}}\left(\sin \left(k R_{\mathrm{h}}\right)-\left(k R_{\mathrm{h}}\right) \cos \left(k R_{\mathrm{h}}\right)\right)
$$

is the Fourier transform of a spherical window of radius $R_{\mathrm{h}}$.

Overdensity peaks can be filtered on specified spatial or mass scales by Gaussian smoothing the random density field [27]

$$
\sigma\left(r_{\mathrm{o}}\right)=\frac{1}{\left(2 \pi R_{\mathrm{f}}^{2}\right)^{3 / 2}} \int \frac{\delta \rho}{\rho}\left(r^{\prime}\right) \exp \left(-\frac{\left|r_{\mathrm{o}}-r^{\prime}\right|^{2}}{2 R_{\mathrm{f}}^{2}}\right) d^{3} r^{\prime}
$$


on a comoving scale $R_{\mathrm{f}}$ centered at $r=r_{\mathrm{o}}$ (for example, $R_{\mathrm{f}}=5 h^{-1} \mathrm{Mpc}$ with a filtered mass of $M_{\mathrm{f}} \sim 10^{15} M_{\odot}$ over cluster scales). $N \sigma$ peaks are generated by sampling different random field realizations to satisfy the condition $\nu=\sigma\left(r_{\mathrm{o}}\right) / \sigma\left(R_{\mathrm{f}}\right)=N$, where $\sigma\left(R_{\mathrm{f}}\right)$ is the rms of Gaussian filtered density fluctuations over a spherical volume of radius $R_{\mathrm{f}}$.

Bertschinger [44] has provided a useful and publicly available package of programs called COSMICS for computing transfer functions, CMB anisotropies, and gaussian random initial conditions for numerical structure formation calculations. The package solves the coupled linearized Einstein, Boltzman, and fluid equations for scalar metric perturbations, photons, neutrinos, baryons, and collisionless dark matter in a background isotropic Universe. It also generates constrained or unconstrained matter distributions over arbitrarily specifiable spatial or mass scales.

Living Reviews in Relativity

http: //www. livingreviews. org/lrr-2001-2 


\section{References}

[1] Abel, T., Anninos, P., Zhang, Y., and Norman, M.L., "Modeling Primordial Gas in Numerical Cosmology", New Astronomy, 2, 181-207, (June, 1997). 4.3, 6.4.2, 6.4.3

[2] Abel, T., Anninos, P., Zhang, Y., and Norman, M.L., "First Structure Formation: I. Primordial Star Forming Regions in hierarchical models", Astrophys. J., 508, 518, (1998). 4.3

[3] Abel, T., Bryan, G.L., and Norman, M.L., "The Formation and Fragmentation of Primordial Molecular Clouds", Astron. Astrophys., 540, 39-44, (2000). 4.3

[4] Abney, M., "hydrodynamic Detonation Instability in Electroweak and QCD Phase Transitions", Phys. Rev. D, 49, 1777-1782, (1994). 3.4

[5] Alcubierre, M., "The appearance of coordinate shocks in hyperbolic formalisms of General Relativity", Phys. Rev. D, 55, 5981-5991, (1997). 6.1 .2

[6] Alcubierre, M., Brügmann, B., Dramlitsch, T., Font, J.A., Papadopoulos, P., Seidel, E., Stergioulas, N., and Takahashi, R., "Towards a Stable Numerical Evolution of Strongly Gravitating Systems in General Relativity: The Conformal Treatments", Phys. Rev. D, 62, 044034, (2000). 6.1 .1

[7] Anninos, P., "Plane-symmetric Cosmology with Relativistic Hydrodynamics", Phys. Rev. D, 58, 064010, (1998). 3.6.1, 6.1.2, 6.1.2, 6.3

[8] Anninos, P., Camarda, K., Massó, J., Seidel, E., Suen, W.-M., and Towns, J., "ThreeDimensional numerical relativity: the evolution of black holes", Phys. Rev. D, 52, 2059-2082, (1995). $\quad 6.1 .2$

[9] Anninos, P., Centrella, J., and Matzner, R., "Nonlinear Solutions for Initial Data in the Vacuum Einstein Equations in plane symmetry", Phys. Rev. D, 39, 2155-2171, (1989). 3.6 .1

[10] Anninos, P., Centrella, J., and Matzner, R., "Nonlinear Wave Solutions to the Planar Vacuum Einstein Equations", Phys. Rev. D, 43, 1825-1838, (1991). 3.6.1

[11] Anninos, P., Centrella, J., and Matzner, R., "Numerical Methods for Solving the Planar Vacuum Einstein Equations", Phys. Rev. D, 43, 1808-1824, (1991). 3.6.1

[12] Anninos, P., and Fragile, P.C., "Nonoscillatory Central Difference and Artificial Viscosity Schemes for Relativistic Hydrodynamics", Astrophys. J. Suppl. Ser., 144, 243-257, (2003). 6.2 .4

[13] Anninos, P., Masso, J., Seidel, E., Suen, W.-M., and Tobias, M., "Dynamics of Gravitational Waves in 3D: Formulations, Methods, and Tests", Phys. Rev. D, 56, 842-858, (1997). 6.1 .1

[14] Anninos, P., Matzner, R., Rothman, T., and Ryan, M., "How does Inflation Isotropize the Universe?", Phys. Rev. D, 43, 3821-3832, (1991). 3.2 .3

[15] Anninos, P., Matzner, R., Tuluie, R., and Centrella, J., "Anisotropies of the Cosmic Background Radiation in a Hot Dark Matter Universe", Astrophys. J., 382, 71-78, (1991). 4.1.4, 4.1 .4

[16] Anninos, P., and McKinney, J., "Relativistic Hydrodynamics of Cosmological Sheets", Phys. Rev. D, 60, 064011, (1999). 3.6.1, 4.6 
[17] Anninos, P., and Norman, M.L., "Hierarchical Numerical Cosmology: Resolving X-Ray Clusters", Astrophys. J., 459, 12-26, (1996). 4.5.3, 6.4.3

[18] Anninos, P., and Norman, M.L., "The Role of Hydrogen Molecules in the Radiative Cooling and Fragmentation of Cosmological Sheets", Astrophys. J., 460, 556-568, (1996). 4.6

[19] Anninos, P., Norman, M.L., and Clarke, D.A., "Hierarchical Numerical Cosmology with Hydrodynamics: Methods and Code Tests", Astrophys. J., 436, 11-22, (1994). 6.4 .3

[20] Anninos, P., Oliveira, S., and Matzner, R., "Fractal structure in the scalar $\lambda\left(\phi^{2}-1\right)^{2}$ theory", Phys. Rev. D, 44, 1147-1160, (1991). 3.3

[21] Anninos, P., Zhang, Y., Abel, T., and Norman, M.L., "Cosmological Hydrodynamics with Multi-Species Chemistry and Nonequilibrium Ionization and Cooling", New Astronomy, 2, 209-224, (June, 1997). 4.3, 6.4.2, 6.4.3

[22] Anninos, W.Y., Norman, M.L., and Anninos, P., "Nonlinear Hydrodynamics of Cosmological Sheets: II. Fragmentation and the Gravitational Cooling and Thin-Shell Instabilities", Astrophys. J., 450, 1-13, (1995). 4.6

[23] Arnowitt, R., Deser, S., and Misner, C.W., "The Dynamics of General Relativity", in Witten, L., ed., Gravitation: An Introduction to Current Research, 227-265, (Wiley, New York, U.S.A., 1962). Related online version (cited on 07 March 2005): http://arxiv.org/abs/gr-qc/0405109. 6.1.1

[24] Bahcall, N.A., Fan, X., and Cen, R., "Constraining $\Omega$ with Cluster Evolution", Astrophys. J., 485, L53-L56, (1997). 4.5.2

[25] Balakrishna, J., Daues, G., Seidel, E., Suen, W.-M., Tobias, M., and Wang, E., "Coordinate Conditions in Three-Dimensional Numerical Relativity", Class. Quantum Grav., 13, L135L142, (1996). $\quad 6.1 .2$

[26] Banyuls, F., Font, J.A., Ibañez, J.M., Marti, J.M., and Miralles, J.A., "Numerical $3+1$ General Relativistic Hydrodynamics: a Local Characteristic Approach", Astrophys. J., 476, 221-231, (1997). $\quad 6.2 .4$

[27] Bardeen, J.M., Bond, J.R., Kaiser, N., and Szalay, A.S., "The Statistics of Peaks of Gaussian Random Fields", Astrophys. J., 304, 15-61, (1986). 6.4.4, 6.4.4

[28] Barreiro, R.B., "The Cosmic Microwave Background: State of the Art", New Astron. Rev., 44, 179-204, (2000). 4.1 .4

[29] Barrett, J.W., Galassi, M., Miller, W.A., Sorkin, R.D., Tuckey, P.A., and Williams, R.M., "A Parallelizable Implicit Evolution Scheme for Regge Calculus", Int. J. Theor. Phys., 36, 815-840, (1997). 6.1.4

[30] Barrow, J.D., "Chaos in the Einstein Equations", Phys. Rev. Lett., 46, 963-966, (1981). 3.1.1

[31] Baumgarte, T.D., and Shapiro, S.L., "On the Numerical Integration of Einstein's Field Equations", Phys. Rev. D, 59, 024007, (1999). 6.1.1

[32] Belinskii, V.A., Lifshitz, E.M., and Khalatnikov, I.M., "Oscillatory Approach to a Singularity Point in the Relativistic Cosmology", Adv. Phys., 19, 525-573, (1970). 3.1 .1

Living Reviews in Relativity

http://www. livingreviews.org//rr-2001-2 
[33] Belinskii, V.A., Lifshitz, E.M., and Khalatnikov, I.M., "Oscillatory Approach to the Singularity Point in Relativistic Cosmology", Sov. Phys. Usp., 13, 745-765, (1971). 3.1.1, 3.1 .1

[34] Bennett, C.L., Halpern, M., Hinshaw, G., Jarosik, N., Kogut, A., Limon, M., Meyer, S.S., Page, L., Spergel, D.N., Tucker, G.S., Wollack, E., Wright, E.L., Barnes, C., Greason, M.R., Hill, R.S., Komatsu, E., Nolta, M.R., Odegard, N., Peiris, H.V., Verde, L., and Weiland, J.L., "First Year Wilkonson Microwave Anistropy Probe (WMAP) Observations: Preliminary Maps and Basic Results", Astrophys. J. Suppl. Ser., 148, 1-27, (2003). 4.1

[35] Berger, B.K., "Comments on the Computation of Liapunov Exponents for the Mixmaster Universe", Gen. Relativ. Gravit., 23, 1385-1402, (1991). 3.1.1

[36] Berger, B.K., "Numerical Investigation of Cosmological Singularities", (December, 1995). URL (cited on 3 September 1997): http://arXiv.org/abs/gr-qc/9512004. 3.1.2, 6.1.3

[37] Berger, B.K., "Numerical Approaches to Spacetime Singularities", Living Rev. Relativity, 5, (2002). URL (cited on 27 October 2004): http://www.livingreviews.org/lrr-2002-1. 3.1.1, 6.1.3

[38] Berger, B.K., Chrusciel, P.T., Isenberg, J.A., and Moncrief, V., "Global Foliations of Vacuum Spacetimes with $T^{2}$ Isometry", Ann. Phys. (N.Y.), 260, 117-148, (1997). 3.1 .2

[39] Berger, B.K., Garfinkle, D., and Strasser, E., "New Algorithm for Mixmaster Dynamics", Class. Quantum Grav., 14, L29-L36, (1997). 6.1 .3

[40] Berger, B.K., and Moncrief, V., "Numerical Investigations of Cosmological Singularities", Phys. Rev. D, 48, 4676-4687, (1993). 6.1.3, 6.1.3

[41] Berger, B.K., and Moncrief, V., "Evidence for an oscillatory singularity in generic U(1) symmetric cosmologies on $T^{3} \times R$ ", Phys. Rev. D, 58, 064023, (1998). 3.1 .2

[42] Berger, B.K., and Moncrief, V., "Signature for local Mixmaster dynamics in U(1) symmetric cosmologies", Phys. Rev. D, 62, 123501, (2000). 3.1 .2

[43] Bernstein, D., Hobill, D.W., and Smarr, L.L., "Black Hole Spacetimes: Testing Numerical Relativity", in Evans, C.R., Finn, L.S., and Hobill, D.W., eds., Frontiers in Numerical Relativity, 57-73, (Cambridge University Press, Cambridge, U.K.; New York, U.S.A., 1989). 6.1 .1

[44] Bertschinger, E., "COSMICS: Cosmological Initial Conditions and Microwave Anisotropy Codes", (June, 1995). URL (cited on 30 August 2000):

http://arXiv.org/abs/astro-ph/9506070. 6.4.4

[45] Bertschinger, E., "Simulations of Structure Formation in the Universe", Annu. Rev. Astron. Astrophys., 36, 599-654, (1998). 4, 6.4.3

[46] Birrell, N.D., and Davies, P.C.W., Quantum Fields in Curved Space, (Cambridge University Press, Cambridge, U.K.; New York, U.S.A., 1982). 6.2 .2

[47] Bond, J.R., Centrella, J., Szalay, A.S., and Wilson, J.R., "Cooling Pancakes", Mon. Not. R. Astron. Soc., 210, 515-545, (1984). 4.6

[48] Bromm, V., Coppi, P.S., and Larson, R.B., "Forming the First Stars in the Universe: The Fragmentation of Primordial Gas", Astrophys. J., 527, L5-8, (1999). 4.3, 6.4.2 
[49] Bryan, G.L., Cen, R., Norman, M.L., Ostriker, J.P., and Stone, J.M., "X-Ray Clusters from a High-Resolution Hydrodynamic PPM Simulation of the Cold Dark Matter Universe", Astrophys. J., 428, 405-418, (1994). 4.5.3

[50] Bryan, G.L., Machacek, M.E., Anninos, P., and Norman, M.L., "Resolving the Ly $\alpha$ Forest", Astrophys. J., 517, 13-30, (1999). 4.4

[51] Bryan, G.L., and Norman, M.L., "A hybrid AMR appplication for cosmology and astrophysics", in Baden, S.B., Chrisochoides, N.P., Gannon, D.B., and Norman, M.L., eds., Structured Adaptive Mesh Refinement (SAMR) Grid Methods, Proceedings of the Workshop held at the Institute for Mathematics and Its Applications, University of Minnesota, on March 12-13, 1997, vol. 117 of The IMA Volumes in Mathematics and its Applications, 165-170, (Springer, New York, U.S.A., 1997). Related online version (cited on 22 August 2000): http://arXiv.org/abs/astro-ph/9710187. 6.4.3

[52] Bryan, G.L., and Norman, M.L., "Statistical Properties of X-ray Clusters: Analytic and Numerical Comparisons", Astrophys. J., 495, 80-99, (1998). 4.5.3

[53] Burd, A.B., Buric, N., and Ellis, G.F.R., "A Numerical Analysis of Chaotic Behavior in Bianchi IX Models", Gen. Relativ. Gravit., 22, 349-363, (1990). 3.1 .1

[54] Cen, R., "A Hydrodynamic Approach to Cosmology - Methodology", Astrophys. J. Suppl. Ser., 78, 341-364, (1992). $\quad 6.4 .2$

[55] Cen, R., Gott, J.R., Ostriker, J.P., and Turner, E.L., "Strong Gravitational Lensing Statistics as a Test of Cosmogonic Scenarios", Astrophys. J., 423, 1-11, (1994). 4.2

[56] Cen, R., and Ostriker, J.P., "X-ray Clusters in a Cold Dark Matter $+\Lambda$ Universe: A Direct, Large-Scale, High Resolution, HydroDynamic Simulation", Astrophys. J., 429, 4-21, (1994). 4.5.3

[57] Centrella, J., and Matzner, R.A., "Plane-Symmetric Cosmologies", Astrophys. J., 230, 311$324,(1979)$. $\quad 3.6 .1$

[58] Centrella, J., and Matzner, R.A., "Colliding Gravitational Waves in Expanding Cosmologies", Phys. Rev. D, 25, 930-941, (1982). 3.6.1

[59] Centrella, J., and Wilson, J.R., "Planar Numerical Cosmology: I. The Differential Equations", Astrophys. J., 273, 428-435, (1983). 3.2.1, 3.6.1, 6.1.2

[60] Centrella, J., and Wilson, J.R., "Planar Numerical Cosmology: II. The Difference Equations and Numerical Tests", Astrophys. J. Suppl. Ser., 54, 229-249, (1984). 3.2.1, 3.6.1, 6.1.2, 6.1 .2

[61] Centrella, J.M., "Nonlinear Gravitational Waves and Inhomogeneous Cosmologies", in Centrella, J.M., ed., Dynamical Spacetimes and Numerical Relativity, Proceedings of the Workshop held at Drexel University, October 7-11, 1985, 123-150, (Cambridge University Press, Cambridge, U.K.; New York, U.S.A., 1986). 3.6 .1

[62] Charlton, J., Anninos, P., Zhang, Y., and Norman, M.L., "Probing Ly $\alpha$ Absorbers in Cosmological Simulations with Double Lines of Sight", Astrophys. J., 485, 26-38, (1997). 4.4

[63] Cornish, N.J., and Levin, J., "Chaos, Fractals and Inflation", Phys. Rev. D, 53, 3022-3032, (1996). $\quad 3.3$ 
[64] Cornish, N.J., and Levin, J., "The Mixmaster Universe is Chaotic", Phys. Rev. Lett., 78, 998-1001, (1997). 3.1 .1

[65] Crone, M.M., Evrard, A.E., and Richstone, D.O., "The Cosmological Dependence of Cluster Density Profiles", Astrophys. J., 434, 402-416, (1994). 4.5.1

[66] da Silva, A.C., Barbosa, D., Liddle, A.R., and Thomas, P.A., "Hydrodynamical Simulations of the Sunyaev-Zel'dovich effect", Mon. Not. R. Astron. Soc., 317, 37, (2000). 4.5 .4

[67] Dave, R., Hernquist, L., Weinberg, D.H., and Katz, N., "Voight Profile Analysis of the Ly $\alpha$ Forest in a Cold Dark Matter Universe", Astrophys. J., 477, 21-26, (1997). 4.4

[68] Efstathiou, G.P., Davis, M., Frenk, C.S., and White, S.D.M., "Numerical Techniques for Large Cosmological N-Body Simulations", Astrophys. J. Suppl. Ser., 57, 241-260, (1985). 6.4.3, 6.4.4

[69] Ellis, G.F.R., and MacCallum, M.A.H., "A Class of Homogeneous Cosmological Models", Commun. Math. Phys., 12, 108, (1969). 2.2

[70] Ellis, G.F.R., and van Elst, H., "Cosmological Models", in Lachièze-Rey, M., ed., Theoretical and Observational Cosmology, Proceedings of the NATO Advanced Study Institute on Theoretical and Observational Cosmology, Cargèse, France, August 17-29, 1998, vol. 541 of NATO Science Series C, (Kluwer, Dordrecht, Netherlands; Boston, U.S.A., 1999). 2.2

[71] Eppley, K., "Pure Gravitational Waves", in Smarr, L.L., ed., Sources of Gravitational Radiation, Proceedings of the Battelle Seattle Workshop, July 24 - August 4, 1978, 275-291, (Cambridge University Press, Cambridge, U.K., 1979). 6.1 .2

[72] Evrard, A.E., "Beyond N-Body: 3D Cosmological Gas Dynamics", Mon. Not. R. Astron. Soc., 235, 911-934, (1988). 6.4 .3

[73] Ferraz, K., Francisco, G., and Matsas, G.E.A., "Chaotic and Nonchaotic Behavior in the Mixmaster Dynamics", Phys. Lett. A, 156, 407-409, (1991). 3.1.1

[74] Flores, R.A., and Primack, J.R., "Cluster Cores, Gravitational Lensing, and Cosmology", Astrophys. J. Lett., 457, L5-L9, (1996). 4.2

[75] Font, J.A., "Numerical Hydrodynamics in General Relativity", Living Rev. Relativity, 6, (2003). URL (cited on 27 October 2004):

http://www.livingreviews.org/lrr-2003-4. $\quad 6.2 .4$

[76] Fragile, P.C., and Anninos, P., "Hydrodynamic Stability of Cosmological Quark-Hadron Phase Transitions", Phys. Rev. D, 67, 103010, (2003). 3.4

[77] Frenk, C.S., White, S.D.M., Bode, P., Bond, J.R., Bryan, G.L., Cen, R., Couchman, H.M.P., Evrard, A.E., Gnedin, N., Jenkins, A., Khokhlov, A.M., Klypin, A., Navarro, J.F., Norman, M.L., Ostriker, J.P., Owen, J.M., Pearce, F.R., Pen, U.-L., Steinmetz, M., Thomas, P.A., Villumsen, J.V., Wadsley, J.W., Warren, M.S., Xu, G., and Yepes, G., "The Santa Barbara Cluster Comparison Project: A Comparison of Cosmological Hydrodynamics Solutions", Astrophys. J., 525, 554-582, (1999). 6.4 .3

[78] Galli, D., and Palla, F., "The Chemistry of the Early Universe", Astron. Astrophys., 335, 403-420, (1998). $\quad 6.4 .2$ 
[79] Garfinkle, D., "Numerical Simulations of generic Singularities", Phys. Rev. Lett., 93, 161101, (2004). URL (cited on 26 October 2004): http://arXiv.org/abs/gr-qc/0312117. 3.1 .2

[80] Gawiser, E., and Silk, J., "The Cosmic Microwave Background Radiation", Phys. Rep., 333, 245-267, (2000). 4.1 .4

[81] Gentle, A.P., and Miller, W.A., "A Fully (3+1)-D Regge Calculus Model of the Kasner Cosmology", Class. Quantum Grav., 15, 389-405, (1998). 6.1.4

[82] Gnedin, N.Y., "Softened Lagrangian Hydrodynamics for Cosmology", Astrophys. J. Suppl. Ser., 97, 231-257, (1995). 6.4 .3

[83] Goldwirth, D.S., and Piran, T., "Inhomogeneity and the Onset of Inflation", Phys. Rev. Lett., 64, 2852-2855, (1990). 3.2 .2

[84] Hawley, J., Smarr, L., and Wilson, J., "A Numerical Study of Nonspherical Black Hole Accretion. II. Finite Differencing and Code Calibration", Astrophys. J. Suppl. Ser., 55, 211246, (1984). 6.2.4

[85] Hawley, J.F., Smarr, L.L., and Wilson, J.R., "A Numerical Study of Nonspherical Black Hole Accretion. I. Equations and Test Problems", Astrophys. J., 277, 296-311, (1984). 6.2.4

[86] Hern, S.D., "Coordinate Singularities in Harmonically-sliced Cosmologies", Phys. Rev. D, 62, 044003, (2000). 6.1 .2

[87] Hern, S.D., and Stewart, J.M., "The Gowdy T3 Cosmologies Revisited", Class. Quantum Grav., 15, 1581-1593, (1998). 3.1.2

[88] Hernquist, L., and Katz, N., "Performance Characteristics of Tree Codes", Astrophys. J. Suppl. Ser., 64, 715-734, (1989). 6.4 .3

[89] Hobill, D., Bernstein, D., Welge, M., and Simkins, D., "The Mixmaster Cosmology as a Dynamical System", Class. Quantum Grav., 8, 1155-1171, (1991). 3.1 .1

[90] Hockney, R.W., and Eastwood, J.W., Computer Simulation Using Particles, (IOP Publishing, Bristol, U.K., 1988). 6.4 .3

[91] Holcomb, K.A., "Vacuum Axisymmetric Cosmologies", in Centrella, J.M., ed., Dynamical Spacetimes and Numerical Relativity, Proceedings of a workshop held at Drexel University, October 7-11, 1985, 187-200, (Cambridge University Press, Cambridge, U.K.; New York, U.S.A., 1986). 3.6 .2

[92] Hollenbach, D., and McKee, C.F., "Molecule Formation and Infrared Emission in Fast Interstellar Shocks: I. Physical Processes", Astrophys. J. Suppl. Ser., 41, 555-592, (1979). 6.4 .2

[93] Hu, W., and Dodelson, S., "Cosmic Microwave Background Anisotropies", Annu. Rev. Astron. Astrophys., 40, 171-216, (2002). 4.1 .4

[94] Hu, W., Scott, D., Sugiyama, N., and White, M., "The effect of Physical Assumptions on the Calculation of Microwave Background Anisotropies", Phys. Rev. D, 52, 5498-5515, (1995). 4.1.3, 4.1.4, 4.1 .4

[95] Huet, P., Kajantie, K., Leigh, R.G., Liu, B.H., and McLerran, L., "Hydrodynamic Stability Analysis of Burning Bubbles in Electroweak Theory and in QCD", Phys. Rev. D, 48, 24772492, (1993). 3.4 
[96] Ignatius, J., Kajantie, K., Kurki-Suonio, H., and Laine, H., "Growth of Bubbles in Cosmological Phase Transitions", Phys. Rev. D, 49, 3854-3868, (1994). 3.4

[97] Isenberg, J.A., and Moncrief, V., "The Existence of Constant Mean Curvature Foliations of Gowdy 3-Torus Spacetimes", Commun. Math. Phys., 86, 485-493, (1982). 6.1.2

[98] Isenberg, J.A., and Moncrief, V., "Asymptotic Behavior of the Gravitational Field and the Nature of Singularities in Gowdy Spacetimes", Ann. Phys. (N.Y.), 199, 84-122, (1990). 3.1 .2

[99] Jiang, O.-S., Levy, D., Lin, C.-T., Osher, S., and Tadmor, E., "High Resolution Nonoscillatory Central Difference Schemes with Nonstaggered Grids for Hyperbolic Conservation Laws", SIAM J. Numer. Anal., 35, 2147-2168, (1998). 6.2 .4

[100] Jiang, O.-S., and Tadmor, E., "Nonoscillatory Central Schemes for Multidimensional Hyperbolic Conservation Laws", SIAM J. Sci. Comput., 19, 1892-1917, (1998). 6.2 .4

[101] Jones, A.W., and Lasenby, A.N., "The Cosmic Microwave Background", Living Rev. Relativity, 1, (1998). URL (cited on 29 August 2000):

http://www.livingreviews.org/lrr-1998-11. 4.1 .3

[102] Kamionkowski, M., and Freese, K., "Instability and Subsequent Evolution of Electroweak Bubbles", Phys. Rev. Lett., 69, 2743-2746, (1992). 3.4, 3.4

[103] Kang, H., Ostriker, J.P., Cen, R., Ryu, D., Hernquist, L., Evrard, A.E., Bryan, G.L., and Norman, M.L., "A Comparison of Cosmological Hydrodynamic Codes", Astrophys. J., 430, 83-100, (1994). $\quad 6.4 .3$

[104] Kolb, E.W., and Turner, M.S., The Early Universe, vol. 69 of Frontiers in Physics, (AddisonWesley, Reading, U.S.A., 1990). 2.2, 3.2.1

[105] Krasiński, A., Inhomogeneous Cosmological Models, (Cambridge University Press, Cambridge, U.K.; New York, U.S.A., 1996). 2.2

[106] Kurki-Suonio, H., Centrella, J.M., Matzner, R.A., and Wilson, J.R., "Inflation from Inhomogeneous Initial Data in a One-Dimensional Back-Reacting Cosmology", Phys. Rev. D, 35, 435-448, (1987). 3.2 .1

[107] Kurki-Suonio, H., Laguna, P., and Matzner, R.A., "Inhomogeneous Inflation: Numerical Evolution", Phys. Rev. D, 48, 3611-3624, (1993). 3.2.5, 6.2.2

[108] Kurki-Suonio, H., and Laine, M., "On Bubble Growth and Droplet Decay in Cosmological Phase Transitions", Phys. Rev. D, 54, 7163-7171, (1996). 3.4

[109] Kurki-Suonio, H., and Matzner, R., "Anisotropy and Cosmic Nucleosynthesis of Light Isotopes Including ${ }^{7}$ Li", Phys. Rev. D, 31, 1811-1814, (1985). 3.5

[110] Kurki-Suonio, H., Matzner, R.A., Centrella, J.M., Rothman, T., and Wilson, J.R., "Inhomogeneous Nucleosynthesis with Neutron Diffusion", Phys. Rev. D, 38, 1091-1099, (1988). 3.5

[111] Liddle, A.R., "An Introduction to Cosmological Inflation", (January, 1999). URL (cited on 11 January 1999):

http://arXiv.org/abs/astro-ph/9901124. 3.2 
[112] Link, B., "Deflagration Instability in the Quark-Hadron Phase Transition", Phys. Rev. Lett., 68, 2425-2428, (1992). 3.4

[113] Lyth, D.H., and Riotto, A., "Particle Physics Models of Inflation and the Cosmological Density Perturbation", Phys. Rep., 314, 1-146, (1999). 3.2

[114] Ma, C.-P., and Bertschinger, E., "Cosmological Perturbation Theory in the Synchronous and Conformal Newtonian Gauges", Astrophys. J., 455, 7-25, (1995). 4.1 .4

[115] Machacek, M.E., Bryan, G.L., Meiksin, A., Anninos, P., Thayer, D., Norman, M.L., and Zhang, Y., "Hydrodynamical Simulations of the Ly $\alpha$ Forest: Model Comparisons", Astrophys. J., 532, 118-135, (2000). 4.4

[116] Matzner, R.A., Shepley, L.C., and Warren, J.B., "Dynamics of $S O(3, R)$-homogeneous cosmologies", Ann. Phys. (N.Y.), 57, 401-460, (1970). 3.1 .1

[117] Meiksin, A., Bryan, G.L., and Machacek, M.E., "Hydrodynamical Simulations of the Ly $\alpha$ forest: Data Comparisons", Mon. Not. R. Astron. Soc., 327, 296, (2001). 4.4

[118] Miralda-Escudé, J., Cen, R., Ostriker, J.P., and Rauch, M., "The Ly $\alpha$ Forest from Gravitational Collapse in the Cold Dark Matter $+\Lambda$ Model", Astrophys. J., 471, 582-616, (1996). 4.4

[119] Misner, C.W., "Mixmaster Universe", Phys. Rev. Lett., 22, 1071-1074, (1969). 3.1 .1

[120] Misner, C.W., Thorne, K.S., and Wheeler, J.A., Gravitation, (W.H. Freeman, San Francisco, U.S.A., 1973). $\quad 3.1 .1$

[121] Moncrief, V., "Finite-Difference Approach to Solving Operator Equations of Motion in Quantum Theory", Phys. Rev. D, 28, 2485-2490, (1983). 6.1 .3

[122] Monerat, G.A., de Oliveira, H.P., and Soares, I.D., "Chaos in Preinflationary FriedmannRobertson-Walker Universes", Phys. Rev. D, 58, 063504, (1998). 3.3

[123] Moser, A.A., Matzner, R.A., and Ryan Jr, M.P., "Numerical Solutions for Symmetric Bianchi Type IX Universes", Ann. Phys. (N.Y.), 79, 558, (1973). 3.1.1

[124] Mukhanov, V.F., Feldman, H.A., and Brandenberger, R.H., "Theory of Cosmological Perturbations", Phys. Rep., 215, 203-333, (1992). 6.4

[125] Navarro, J.F., Frenk, C.S., and White, S.D.M., "A Universal Density Profile from Hierarchical Clustering", Astrophys. J., 490, 493-508, (1997). 4.5.1

[126] Norman, M.L., and Winkler, K.-H.A., "Why Ultrarelativistic Numerical Hydrodynamics is Difficult", in Winkler, K.-H.A., and Norman, M.L., eds., Astrophysical Radiation Hydrodynamics, Proceedings of the NATO Advanced Research Workshop, Garching, Germany, August 2-13, 1982, vol. 188 of NATO ASI Series C, 449-475, (Reidel, Dordrecht, Netherlands; Boston, U.S.A., 1986). $\quad 6.2 .4$

[127] Ostriker, J.P., and Gnedin, N.Y., "Reheating of the Universe and Population III", Astrophys. J., 472, L63-L67, (1996). 4.3

[128] Ostriker, J.P., and Steinhardt, P.J., "Cosmic Concordance", (May, 1995). URL (cited on 5 September 2000):

http://arXiv.org/abs/astro-ph/9505066. 2.2 
[129] Ove, R., "Numerical Investigations of Cosmic Censorship on $T^{3} x R$ ", in Centrella, J.M., ed., Dynamical Spacetimes and Numerical Relativity, Proceedings of a workshop held at Drexel University, October 7-11, 1985, 201-235, (Cambridge University Press, Cambridge, U.K.; New York, U.S.A., 1986). 3.6.2

[130] Owen, J.M., Villumsen, J.V., Shapiro, P.R., and Martel, H., "Adaptive Smoothed Particle Hydrodynamics: Methodology II", Astrophys. J. Suppl. Ser., 116, 155-209, (1998). 6.4 .3

[131] Padmanabhan, T., Structure Formation in the Universe, (Cambridge University Press, Cambridge, U.K.,; New York, U.S.A., 1993). 4, 4.1.4

[132] Peebles, P.J.E., Principles of Physical Cosmology, Princeton Series in Physics, (Princeton University Press, Princeton, U.S.A., 1993). 4

[133] Peebles, P.J.E., and Ratra, B., "The Cosmological Constant and Dark Energy", Rev. Mod. Phys., 75, 559-606, (2003). 2.2

[134] Quilis, V., Ibañez, J.M., and Saez, D., "A Multidimensional Hydrodynamic Code for Structure Evolution in Cosmology", Astrophys. J., 469, 11-25, (1996). 6.4 .3

[135] Quirk, J.J., "A Contribution to the Great Riemann Solver Debate", Int. J. Numer. Meth. Fluids, 18, 555-574, (1994). 6.2 .4

[136] Reula, O.A., "Hyperbolic Methods for Einstein's Equations", Living Rev. Relativity, 1, (1998). URL (cited on 29 August 2000):

http://www.livingreviews.org/lrr-1998-3. 6.1 .1

[137] Rezzolla, L., "Stability of Cosmological Detonation Fronts", Phys. Rev. D, 54, 1345-1358, (1996). 3.4

[138] Rezzolla, L., Miller, J.C., and Pantano, O., "Evaporation of Quark Drops During the Cosmological Quark-Hadron Transition", Phys. Rev. D, 52, 3202-3213, (1995). 3.4

[139] Romero, J.V., Ibañez, J.M., Martí, J.M., and Miralles, J.A., "A New Spherically Symmetric General Relativistic Hydrodynamical Code”, Astrophys. J., 462, 839-854, (1996). 6.2 .4

[140] Rothman, T., and Matzner, R.A., "Nucleosynthesis in Anisotropic Cosmologies Revisited", Phys. Rev. D, 30, 1649-1668, (1984). 3.5

[141] Ryan Jr, M.P., and Shepley, L.C., Homogeneous Relativistic Cosmologies, Princeton Series in Physics, (Princeton University Press, Princeton, U.S.A., 1975). 2.2, 3.1.2

[142] Ryu, D., Ostriker, J.P., Kang, H., and Cen, R., "A Cosmological Hydrodynamic Code Based on the Total Variation Diminishing Scheme", Astrophys. J., 414, 1-19, (1993). 6.4.3

[143] Schneider, P., Ehlers, J., and Falco, E.E., Gravitational Lenses, Astronomy and Astrophysics Library, (Springer, Berlin, Germany; New York, U.S.A., 1992). 4.2

[144] Shapiro, P.R., and Kang, H., "Hydrogen Molecules and the Radiative Cooling of Pregalactic Shocks", Astrophys. J., 318, 32-65, (1987). 6.4 .2

[145] Shapiro, P.R., and Struck-Marcell, C., "Pancakes and the Formation of Galaxies in a Universe Dominated by Collisionless Particles", Astrophys. J. Suppl. Ser., 57, 205-239, (1985). 4.6

[146] Shibata, M., "Fully general relativistic simulation of merging binary clusters - Spatial gauge condition -", Prog. Theor. Phys., 101, 1199-1233, (1999). 6.1 .2 
[147] Shibata, M., and Nakamura, T., "Evolution of Three-Dimensional Gravitational Waves: Harmonic Slicing Case", Phys. Rev. D, 52, 5428-5444, (1995). 6.1.1

[148] Shinkai, H., and Maeda, K., "Can Gravitational Waves Prevent Inflation", Phys. Rev. D, 48, 3910-3913, (1993). 3.2 .4

[149] Spergel, D.N., Verde, L., Peiris, H.V., Komatsu, E., Nolta, M.R., Bennett, C.L., Halpern, M., Hinshaw, G., Jarosik, N., Kogut, A., Limon, M., Meyer, S.S., Page, L., Tucker, G.S., Weiland, J.L., Wollack, E., and Wright, E.L., "First Year Wilkonson Microwave Anistropy Probe (WMAP) Observations: Determination of Cosmological Parameters", Astrophys. J. Suppl. Ser., 148, 175-194, (2003). 4.1

[150] Springel, V., White, M., and Hernquist, L., "Hydrodynamic Simulations of the SunyaevZel'dovich Effect(s)", Astrophys. J., 549, 681-687, (2001). 4.5.4

[151] Stancil, P.C., Lepp, S., and Dalgarno, A., "The Deuterium Chemistry of the Early Universe", Astrophys. J., 509, 1-10, (1998). 6.4 .2

[152] Stebbins, A., "The CMBR Spectrum", (May, 1997). URL (cited on 29 August 2003): http://arXiv.org/abs/astro-ph/9705178. 4.1.4

[153] Susa, H., and Umemura, M., "Formation of Primordial Galaxies Under UV Background Radiation", Astrophys. J., 537, 578-588, (2000). 4.6

[154] Tegmark, M., Zaldarriaga, M., and Hamilton, A.J.S., "Towards a refined cosmic concordance model: joint 11-parameter constraints from CMB and large-scale structure", Phys. Rev. D, 63, 043007, (2001). $\quad 2.2$

[155] Thomas, P.A., Colberg, J.M., Couchman, H.M.P., Efstathiou, G.P., Frenk, C.S., Jenkins, A.R., Nelson, A.H., Hutchings, R.M., Peacock, J.A., Pearce, F.R., and White, S.D.M., "The structure of galaxy clusters in various cosmologies", Mon. Not. R. Astron. Soc., 296(4), 1061-1071, (June, 1998). Related online version (cited on 3 September 1997):

http://arXiv.org/abs/astro-ph/9707018. 4.5.1

[156] Tuluie, R., Laguna, P., and Anninos, P., "Cosmic Microwave Background Anisotropies from the Rees-Sciama Effect in $\Omega_{0} \leq 1$ Universes", Astrophys. J., 463, 15-25, (1996). 4.1.4

[157] Tuluie, R., Matzner, R.A., and Anninos, P., "Anisotropies of the Cosmic Background Radiation in a Reionized Universe", Astrophys. J., 446, 447-456, (1995). 4.1.4

[158] Wainwright, J., and Ellis, G.F.R., Dynamical Systems in Cosmology, (Cambridge University Press, Cambridge, U.K.; New York, U.S.A., 1997). 2.2

[159] Warren, M.S., and Salmon, J.K., "A Portable Parallel Particle Program", Computer Phys. Commun., 87, 266-290, (1995). $\quad 6.4 .3$

[160] Weaver, M., Isenberg, J.A., and Berger, B.K., "Mixmaster Behavior in Inhomogensous Cosmological Spacetimes", Phys. Rev. Lett., 80, 2984-2987, (1998). 3.1 .2

[161] Weinberg, S., Gravitation and Cosmology: Principles and Applications of the General Theory of Relativity, (Wiley, New York, U.S.A., 1972). 6.2 .3

[162] Wilson, J.R., "A Numerical Method for Relativistic Hydrodynamics", in Smarr, L.L., ed., Sources of Gravitational Radiation, Proceedings of the Battelle Seattle Workshop, July 24 August 4, 1978, 423-445, (Cambridge University Press, Cambridge, U.K.; New York, U.S.A., 1979). $\quad 3.6 .1,6.2 .4,6.2 .4$

Living Reviews in Relativity

http://www. livingreviews . org/lrr-2001-2 
[163] York Jr, J.W., "Kinematics and Dynamics of General Relativity", in Smarr, L.L., ed., Sources of Gravitational Radiation, Proceedings of the Battelle Seattle Workshop, July 24 - August 4, 1978, 83-126, (Cambridge University Press, Cambridge, U.K.; New York, U.S.A., 1979). 6.1.4, 6.3

[164] Yoshida, N., Bromm, V., and Hernquist, L., "The Era of Massive Population III Stars: Cosmological Implications and Self-Termination", Astrophys. J., 605, 579-590, (2004). 4.3

[165] Zel'dovich, Y.B., "Gravitational Instability: An Approximate Theory for Large Density Perturbations", Astron. Astrophys., 5, 84-89, (1970). 3.6.1, 4.6, 6.4.4

[166] Zhang, Y., Norman, M.L., Anninos, P., and Meiksin, A., "Spectral Analysis of the Ly $\alpha$ Forest in a Cold Dark Matter Cosmology", Astrophys. J., 485, 496-516, (1997). 4.4 